\title{
Heavy baryon wave functions, Bakamjian-Thomas approach to form factors, and observables in $\Lambda_{b} \rightarrow \Lambda_{c}\left(\frac{1}{2}\right) \ell \bar{\nu}$ transitions
}

\author{
D. Bečirević, ${ }^{1}$ A. Le Yaouanc, ${ }^{1}$ V. Morénas, ${ }^{2}$ and L. Oliver ${ }^{1}$ \\ ${ }^{1}$ Laboratoire de Physique Théorique, Unité Mixte de Recherche UMR 8627-CNRS, \\ Université de Paris XI, Bâtiment 210, 91405 Orsay Cedex, France \\ ${ }^{2}$ Laboratoire de Physique de Clermont-Ferrand, Unité Mixte de Recherche UMR 6533-CNRS, \\ Campus des Cézeaux 4 Avenue Blaise Pascal, TSA 60026, CS 60026, 63178 Aubière Cedex, France
}

(Received 25 June 2020; accepted 24 September 2020; published 23 November 2020)

\begin{abstract}
Motivated by the calculation of observables in the decays $\Lambda_{b} \rightarrow \Lambda_{c}\left(\frac{1}{2} \pm\right) \ell \bar{\nu}$, we present a calculation of form factors in the quark model. Our scheme combines a spectroscopic model with the internal wave functions and the Bakamjian-Thomas (BT) relativistic formalism to get the wave functions in motion. In the heavy quark limit, the current matrix elements provide the Isgur-Wise (IW) function. This limit is covariant, satisfies a large set of sum rules, and has been successfully applied to mesons. On the other hand, for baryons, we meet difficulties using standard spectroscopic models. This leads us to propose a phenomenological model, a Q-pointlike-diquark model, nonrelativistic, with harmonic oscillator forces, giving both a reasonable low-lying spectrum and the expected slope of the IW function. To begin, we extract this slope from lattice QCD data and find it to be around $\rho_{\Lambda}^{2} \sim 2$, that we use as a guideline. We are not able to reproduce the right $\rho_{\Lambda}^{2}$ using certain typical standard linear + Coulomb potential models, both with three quarks $Q q q$ or in a Q-pointlike-diquark picture. These difficulties seem to derive from the high sensitivity of $\rho_{\Lambda}^{2}$ to the structure of the light quark subsystem in a relativistic scheme. Finally, we present our model, and fixing its parameters to yield the correct spectrum and $\rho_{\Lambda}^{2} \sim 2$, we apply it to the calculation of observables. By studying Bjorken sum rule, we show that the inelastic IW function is large, and therefore, the transitions $\Lambda_{b} \rightarrow \Lambda_{c}\left(\frac{1}{2}^{-}, \frac{3}{2}^{-}\right) \ell \bar{\nu}$ could be studied at LHCb. Interestingly, some observables in the $\tau$ case present zeroes for specific values of $q^{2}$ that could be tests of the Standard Model.
\end{abstract}

DOI: 10.1103/PhysRevD.102.094023

\section{INTRODUCTION}

Possible physics beyond the Standard Model (SM), suggesting lepton flavor universality violation (LFUV), has been pointed out by data of different experiments on $\bar{B} \rightarrow D^{(*)} \ell \nu$ [1-3], summarized in [4]. That has attracted much attention in the Standard Model (SM) and also in models for new physics (NP) [5-12], in particular, following lattice calculations of the form factors in the SM [13,14].

With the intention of providing predictions for observables in $\Lambda_{b} \rightarrow \Lambda_{c}^{(*)} \ell \bar{\nu}$, we consider the quark model, which can describe the ground state and also the excitations, not calculated still in lattice QCD. Moreover, we use the BT relativistic framework, that yields a Lorentz invariant IsgurWise (IW) function in terms of internal hadron wave

Published by the American Physical Society under the terms of the Creative Commons Attribution 4.0 International license. Further distribution of this work must maintain attribution to the author(s) and the published article's title, journal citation, and DOI. Funded by SCOAP. functions, deduced from a quark model spectroscopic Hamiltonian describing the states at rest.

We have used this guideline in the meson case $\bar{B} \rightarrow D^{(*)} \ell \bar{\nu}$, for the ground state [15] and orbitally excited $D$ mesons [16]. In the meson case, we did use the spectroscopic Hamiltonian of Godfrey and Isgur (GI), that describes a wealth of meson data [17]. In this way, it was obtained a reasonable and theoretically founded description of IW functions, both elastic and inelastic [18].

The BT scheme is an approach to hadron motion, which can be combined with any internal wave function. In quark model calculations $[19,20]$, the spectroscopic model providing these wave functions could be either nonrelativistic, as in [19], or relativistic, as in Pervin et al. [20]. But whatever the type of spectroscopic equation, both schemes apply the nonrelativistic treatment for the hadron motion. In [19], although a careful calculation of the spectrum and wave functions is done, a very small IW slope is found $\rho_{\Lambda}^{2} \simeq 0.6-1$, instead of $\rho_{\Lambda}^{2} \simeq 2$ for the $\Lambda_{b}$, as indicated by our fit below to lattice QCD [13] and LHCb data [21].

As has been shown in the meson case [15,18,22], the BT calculation gives a large enhancement for the IW slope with 
respect to the nonrelativistic calculation with the same internal wave functions. This is due to the Lorentz transformation of the spatial arguments of the wave function in motion. This effect gives, with respect to the nonrelativistic slope $\left(\rho_{\mathrm{NR}}^{2}=\frac{1}{2} m^{2} R^{2}\right.$ for a Gaussian), an additional contribution that is independent of the wave function shape and parameters and very large since it is roughly $\delta \rho^{2} \simeq 0.75$ for a model with a scalar light quark, and $\delta \rho^{2} \simeq 1$ for a meson [22], in agreement with a Bethe-Salpeter approach [23].

We can write an explicit formula for the slope in the BT scheme for a $Q-\bar{q}$ Gaussian wave function $\exp \left(-R^{2} p^{2} / 2\right)$ for a $Q-\bar{q}$ bound state. The product of the initial and final wave functions with Lorentz transformation along $O z$ gives $\exp \left[-R^{2}\left(w\left(p^{z}\right)^{2}+\frac{w+1}{2}\left(p^{T}\right)^{2}+\right.\right.$ $\left.\frac{w-1}{2} m^{2}\right)$ ]. Integrating over $p$, and expanding around $w=1$, one finds the slope, giving the result $\rho^{2}=\frac{1}{2} m^{2} R^{2}+1$. The +1 is the enhancement with respect to the nonrelativistic result, which is the first term. The Jacobian expanded in terms of the internal velocity gives -0.25 , whence the final enhancement $0.75+\mathcal{O}\left(v^{2} / c^{2}\right)$ for a $j^{P}=0^{+}$light cloud. On the other hand, for a meson, there is the contribution $+\frac{1}{4}$ from the heavy quark current, and the slope is around 1 .

For baryons, the structure of the three-quark wave function and the BT expression for the IW function makes this enhancement effect difficult to evaluate, as it is strongly dependent on this structure. The general expectation is that the enhancement of $\rho^{2}$ should be larger than for mesons. In the simple case of wave functions factorized in $\left|\vec{r}_{2}\right|,\left|\vec{r}_{3}\right|$, one would have an enhancement for two light quarks twice the one for one light quark, $\delta \rho^{2} \simeq 2 \times 0.75=1.5$, pointing naturally towards $\rho_{\Lambda}^{2} \simeq 2$ or more. But it could be much larger for a wave function of the type of [20]. One observes a very strong dependence of $\rho_{\Lambda}^{2}$ on the structure of the wave function. For instance, for a Gaussian in the relative $\vec{\lambda}, \vec{\rho}$ coordinates, it depends strongly on the ratio $R_{\rho} / R_{\lambda}$ and may acquire much too large values.

Another important feature of the BT approach is that it implements automatically the heavy quark effective theory (HQET) sum rules like Bjorken's or the curvature sum rules, which help to constrain the contributions of higher states.

Trying to apply this scheme to heavy baryons, we have found problems.

There are several quark model approaches that could provide the required internal wave functions. Among the standard linear + Coulomb potentials, there is work parallel to GI for mesons, the relativistic Hamiltonian of Capstick and Isgur for the $Q q q$ system [24], which is a rather complicated model that reproduces a large spectrum of states, but for which it is not easy to obtain the wave functions. Second, the work of Albertus et al. [19], using a nonrelativistic kinetic energy, writes explicitly the wave functions, but these are not easy to use. Third, there is the quark model study of Pervin et al. for $\Lambda_{Q}$ baryons [20], which is more manageable, to which we refer now.
In the present paper, we have computed the IW function $\Lambda_{b} \rightarrow \Lambda_{c}$ in terms of a generic internal $Q q q$ wave function. Then, we have used an internal wave function given by [20] to compute numerically the IW function and the slope. As pointed out below, using the parameters of Pervin et al. [20], we have found a slope $\rho_{\Lambda}^{2} \simeq 4$. This is much larger than the estimate by LHCb, $\rho_{\Lambda}^{2} \simeq 1.8$ [21], and the value that follows from lattice QCD calculations by Detmold et al. [13], that gives $\rho_{\Lambda}^{2} \simeq 2$, as we show below. We identify the origin of this large value of the slope, and we comment on the related work by Cardarelli and Simula in the light front formulation of the BT approach [25].

Then, we turn to the simpler scheme of a quark-diquark model, a bound state of a heavy quark and a color triplet pointlike diquark. This model has been widely used to compute heavy baryon spectra and heavy baryon form factors [26]. Concerning the spectrum, there is the interesting paper by Bing Chen et al. [27], a nonrelativistic model with QCD-inspired potential, that also presents problems for the IW slope.

On the other hand, within the quark-diquark scheme, but renouncing to QCD-inspired potentials, a simple nonrelativistic harmonic oscillator model can be adjusted to give reasonable level spacings, and one can get also the IW slope in the BT scheme $\rho_{\Lambda}^{2} \simeq 2$. In this paper, we will adopt, for the moment, this simple model for the internal wave functions in view of the computation of $\Lambda_{b} \rightarrow \Lambda_{c}^{(*)} \ell \bar{\nu}$ observables. Recently, the Mainz group has issued a paper [28] on some observables that could be useful to test LFUV in $\Lambda_{b} \rightarrow \Lambda_{c}\left(\frac{1}{2}^{ \pm}, \frac{3}{2}^{-}\right) \ell \bar{\nu}$ transitions. However, as our approach is different, we still present our results and compare with their work and other related literature.

In Sec. II, we present a fit to the lattice QCD data, that yields a slope of the IW function $\rho_{\Lambda}^{2} \simeq 2$ and quotes the value given by LHCb. In Sec. III, we expose the numerical problem that we find on computing the IW slope for $Q q q$ baryons with the wave functions of Ref. [20], and we trace back the origin of this difficulty using a Gaussian wave function, in the spirit of Cardarelli and Simula [25]. We turn to the quark-diquark model in Sec. IV, we compute the IW functions for the elastic and inelastic cases in the BT scheme from the wave functions of the Bing Chen et al. Hamiltonian [27], and we find a too small slope compared to the lattice result. In Sec. V, in front of these difficulties, to compute the observables, we renounce to models with QCD-inspired potentials and use quark-diquark wave functions deduced from a nonrelativistic harmonic oscillator model, adjusted to give the desired $\rho_{\Lambda}^{2} \simeq 2$. In Appendix A, we define the baryon form factors in different conventions, in Appendix B, we give some details of the involved calculation of the $Q q q$ elastic IW function in the BT scheme, in Appendix C, we compute the elastic and inelastic quark-diquark IW functions in the BT scheme, in Appendix D, we make explicit the quark-diquark wave 
functions within the Bing Chen et al. scheme, and finally, in Appendix E, we give the expressions for the helicity amplitudes and the observables as formulated by the Mainz group, that we have used for our applications in Sec. V.

\section{LHCb MEASUREMENT OF THE $d \Gamma / d q^{2}$ SHAPE, LATTICE FORM FACTORS, AND SLOPE OF THE IW FUNCTION}

\section{A. LHCb measurement of the $d \Gamma / d q^{2}$ shape}

The differential rate of the decay $\Lambda_{b} \rightarrow \Lambda_{c}+\ell^{-}+\bar{\nu}_{\ell}$ writes, for $m_{\ell}=0$ and the heavy quark limit form factors,

$$
\begin{aligned}
\frac{d \Gamma}{d w}= & \frac{G_{F}^{2}}{12 \pi^{3}}\left|V_{c b}\right|^{2} m_{\Lambda_{b}}^{2} m_{\Lambda_{c}}^{3} \sqrt{w^{2}-1} \\
& \times\left[3 w\left(1-2 r w+r^{2}\right)+2 r\left(w^{2}-1\right)\right]\left|\xi_{\Lambda}(w)\right|^{2},
\end{aligned}
$$

where $r=m_{\Lambda_{c}} / m_{\Lambda_{b}}$.

Formula (1) is an approximation to the more general formula (10) given for the decay rate in Appendix 1 of the $\mathrm{LHCb}$ paper [21] in terms of the different form factors $f_{0}\left(q^{2}\right), f_{+}\left(q^{2}\right), f_{\perp}\left(q^{2}\right), g_{0}\left(q^{2}\right), g_{+}\left(q^{2}\right), g_{\perp}\left(q^{2}\right)$.

The LHCb fit relies on the approximation that all these form factors are approximated by the first order term in the $O\left(1 / m_{Q}\right)$ expansion, $f_{0}\left(q^{2}\right), \ldots g_{\perp}\left(q^{2}\right) \simeq \xi_{\Lambda}(w)$. However, the $O\left(1 / m_{Q}\right)$ corrections to these form factors contain also terms of the type $\bar{\Lambda} \xi_{\Lambda}(w) / m_{Q}$, as we can see by the inspection of our Appendix A.

Therefore, here $\xi_{\Lambda}(w)$ is an approximation to the IW function, with unspecified $1 / m_{Q}$ errors. In the next subsection, we try to extract the IW function from the form factors calculated in lattice QCD by taking into account these $1 / m_{Q}$ corrections.

With the "dipole" ansatz,

$$
\xi_{\Lambda}(w)=\left(\frac{2}{w+1}\right)^{2 \rho_{\mathrm{dip}}^{2}} .
$$

LHCb finds the value of this $\rho_{\text {dip }}^{2}$ parameter [21],

$$
\rho_{\text {dip }}^{2}=-\xi_{\Lambda}^{\prime}(1)=1.82 \pm 0.03,
$$

and the curvature,

$$
\sigma_{\text {dip }}^{2}=\xi_{\Lambda}^{\prime \prime}(1)=4.22 \pm 0.12
$$

Of course, the very small errors in Eqs. (3) and (4) are not to be taken as the actual errors on the real IW slope and curvature.

The main reason for adopting the shape (2) is that it satisfies a number of theorems in the heavy quark limit of HQET that constrain the successive zero recoil derivatives of the baryon IW function $[29,30]$. In particular, there is the bound on the curvature,

$$
\sigma_{\Lambda}^{2} \geq \frac{3}{5}\left[\rho_{\Lambda}^{2}+\left(\rho_{\Lambda}^{2}\right)^{2}\right] .
$$

It has been established [30] that the "dipole" form (2), that depends on a single parameter, satisfies these constraints provided that $\rho_{\Lambda}^{2} \geq \frac{1}{4}$.

\section{B. Fits to lattice data on form factors}

Early studies of the $\Lambda_{b} \rightarrow \Lambda_{c} \ell \nu$ lattice form factors in the quenched approximation were provided in Refs. [31,32]. In the former study, a value was given for the slope of the IW function, $\rho_{\Lambda}^{2} \simeq 2.4$ with a $15 \%$ error, showing no dependence on the heavy quark masses and a value was also obtained for the HQET parameter $\bar{\Lambda} \simeq 0.75$ with a $20 \%$ error.

A great wealth of new precise data in lattice QCD has been obtained recently by W. Detmold et al. [13], which have given results for all the form factors entering in the process $\Lambda_{b} \rightarrow \Lambda_{c} \ell \bar{\nu}_{\ell}$ within the Standard Model.

Our aim is now to try to extract information on the slope of the IW function $\xi_{\Lambda}(w)$ and other parameters, $\bar{\Lambda}$ and the heavy quark masses $m_{Q}(Q=b, c)$, from these lattice calculations, that are summarized in Fig. 12 of Ref. [13].

We adopt a simple HQET model, keeping the form factors up to first order in $1 / m_{Q}$ included, as given in the formulas of Appendix A.

Inspection of the formulas of Appendix A shows that, at this first order, besides the dependence of the form factors on the IW function $\xi_{\Lambda}(w)$, on the heavy quark masses $m_{Q}(Q=b, c)$ and on the HQET parameter $\bar{\Lambda}$, there is another subleading function $A(w)$ that, due to Luke's theorem [33], must vanish at $w=1$. For this function, since the domain in $w$ is not large, we adopt the parametrization,

$$
A(w)=A^{\prime}(1)(w-1) .
$$

Moreover, we will adopt the explicit "dipole" form (2) for the leading IW function.

\section{The IW function slope from lattice form factors}

In the approximation that we adopt, HQET up to the first order in $1 / m_{Q}$ included, there are two quantities that isolate the IW function, where all dependence on $\bar{\Lambda}$ and the parameter $A^{\prime}(1)$ defined by (6) cancels. These quantities are differences of ratios that, up to $O\left(1 / m_{Q}^{2}\right)$ corrections, are identical to the IW function $\xi_{\Lambda}(w)$,

$$
\begin{gathered}
R_{1}(w)=\frac{w+1}{2} \frac{f_{\perp}(w)-g_{\perp}(w)}{f_{\perp}(1)-g_{\perp}(1)} \\
R_{2}(w)=\frac{f_{\perp}(w)-f_{+}(w)}{f_{\perp}(1)-f_{+}(1)} .
\end{gathered}
$$

Inspection of the formulas of Appendix A shows indeed that these ratios do not depend on $\bar{\Lambda}$ and on the parameter $A^{\prime}(1)$, that cancel in these quantities, 


$$
\begin{aligned}
& R_{1}(w)=\xi_{\Lambda}(w)+O\left(1 / m_{Q}^{2}\right) \\
& R_{2}(w)=\xi_{\Lambda}(w)+O\left(1 / m_{Q}^{2}\right) .
\end{aligned}
$$

To have information on the IW function, we will use the $z$-expansion parametrization in [13], that we will fit with our HQET model of form factors, that includes up to $O\left(1 / m_{Q}\right)$ corrections, made explicit in Appendix A. The lattice data are parametrized by the $z$ expansion [34] for each form factor,

$$
\begin{aligned}
f\left(q^{2}\right) & =\frac{1}{1-\frac{q^{2}}{\left(m_{\mathrm{pole}}^{f}\right)^{2}}}\left[a_{0}^{f}+a_{1}^{f} z^{f}\left(q^{2}\right)+\cdots\right], \\
z^{f}\left(q^{2}\right) & =\frac{\sqrt{t_{+}^{f}-q^{2}}-\sqrt{t_{+}^{f}-t_{0}}}{\sqrt{t_{+}^{f}-q^{2}}-+\sqrt{t_{+}^{f}-t_{0}}},
\end{aligned}
$$

where $t_{0}=\left(m_{\Lambda_{b}}-m_{\Lambda_{c}}\right)^{2}, \quad m_{\text {pole }}^{f}$ and $t_{+}^{f}$ are given in Table VII, $a_{0}^{f}, a_{1}^{f}$ up to $O(z)$ in Table VIII, and $a_{0}^{f}, a_{1}^{f}, a_{2}^{f}$ up to $O\left(z^{2}\right)$ in Table $\mathrm{X}$ of [13].

We do not pretend to make a fit on the two ratios (7), (8) with their errors. We just take the z-expansion central values at face value, to see if for the two ratios we find reasonable consistent values for the IW function slope $\rho_{\Lambda}^{2}$, using both expansions up to $O(z)$ and up to $O\left(z^{2}\right)$. For the IW function, we adopt the "dipole" parametrization (2), that satisfies the rigorous results that constrain the successive zero recoil derivatives of the baryon IW function $[29,30]$.

To perform the fits, we select a number of points ("synthetic data points"), $q^{2}=0 ., 2 ., 4 ., 6 ., 8 ., 10 ., 11 ., q_{\max }^{2}$, of the $z$ expansions of the lattice fits for the form factors, up to first order and up to second order in $z$, and we use the Mathematica package FindFit to fit our HQET model of form factors of Appendix A, that includes $O\left(1 / m_{Q}\right)$ corrections. For the IW function $\xi_{\Lambda}(w)$, we consider the domain

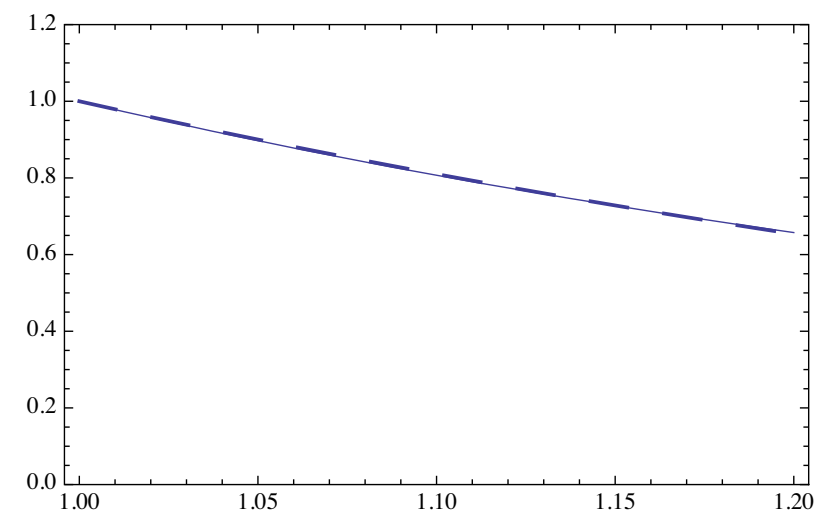

$1 \leq w \leq 1.2$, where there are data points measured on the lattice. For the individual form factors, we will consider below the $z$ expansions and the fits for the whole phase space.

The results for the IW function are given in Figs. 1 and 2. From the ratio $R_{1}(w)$, we get from the fit in the region $1 \leq w \leq 1.2, \rho_{\Lambda}^{2} \simeq 2.20$ for the first order expansion in $z$, to be compared with the true slope of the IW function (7) $-\xi_{\Lambda}^{\prime}(1)=2.11$, and $\rho_{\Lambda}^{2} \simeq 2.03$ for the second order in $z$, to be compared with the true slope $-\xi_{\Lambda}^{\prime}(1)=1.99$. On the other hand, from the ratio $R_{2}(w)$, we get results that are close in both cases, $\rho_{\Lambda}^{2} \simeq 2.25$, compared to the true slope $-\xi_{\Lambda}^{\prime}(1)=2.16$ at first order in $z$, and $-\xi_{\Lambda}^{\prime}(1)=2.21$ at second order.

We can safely conclude that the slope is consistent with the following ranges obtained from the fit. For the first order $z$ expansion, we get the domain,

$$
\rho_{\Lambda}^{2} \simeq 2.20-2.24
$$

while for the $z^{2}$ order, we obtain the range,

$$
\rho_{\Lambda}^{2} \simeq 2.03-2.25
$$

Although our fits are somewhat naive, from (11), (12), we can safely conclude that the data on $\Lambda_{b} \rightarrow \Lambda_{c}$ form factors [13] can be described in HQET up to $O\left(1 / m_{Q}\right)$ included, with the slope of the "dipole" form for the IW function (2),

$$
\rho_{\Lambda}^{2} \simeq 2.15 \pm 0.10 \text {. }
$$

\section{Fits to the different form factors}

We do not want to make an overall fit on the whole set of form factors with their errors (errors that we do not master) but just to study individually each form factor of Fig. 12 of [13] by making a fit to the central values of these domains, given by the $z$ expansion up to order $z$.

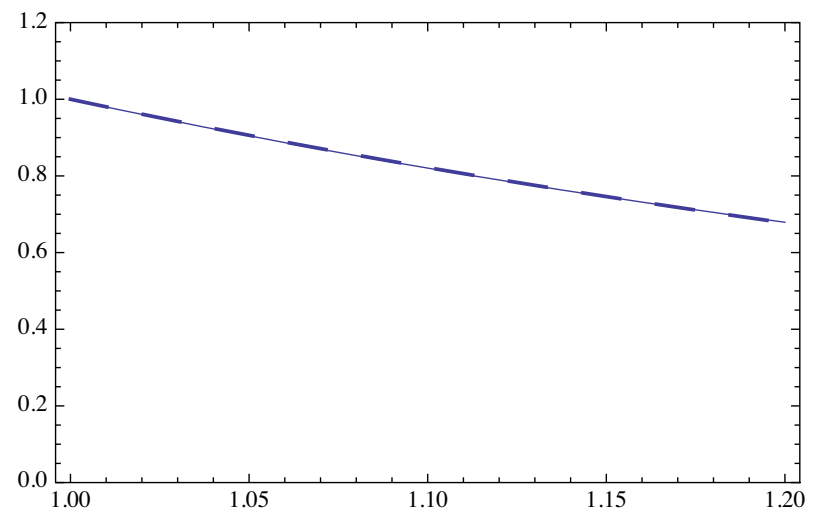

FIG. 1. Fits to the ratio $R_{1}(w) \simeq \xi(w)$ (7), (9) with the Isgur-Wise function (2) (continuous curve) using the parametrization of the lattice data up to first order in the $z$ expansion (dashed curve, left) that yields the slope $\rho_{\Lambda}^{2} \simeq 2.20$, and up to second order (dashed curve, right), that gives $\rho_{\Lambda}^{2} \simeq 2.03$. 

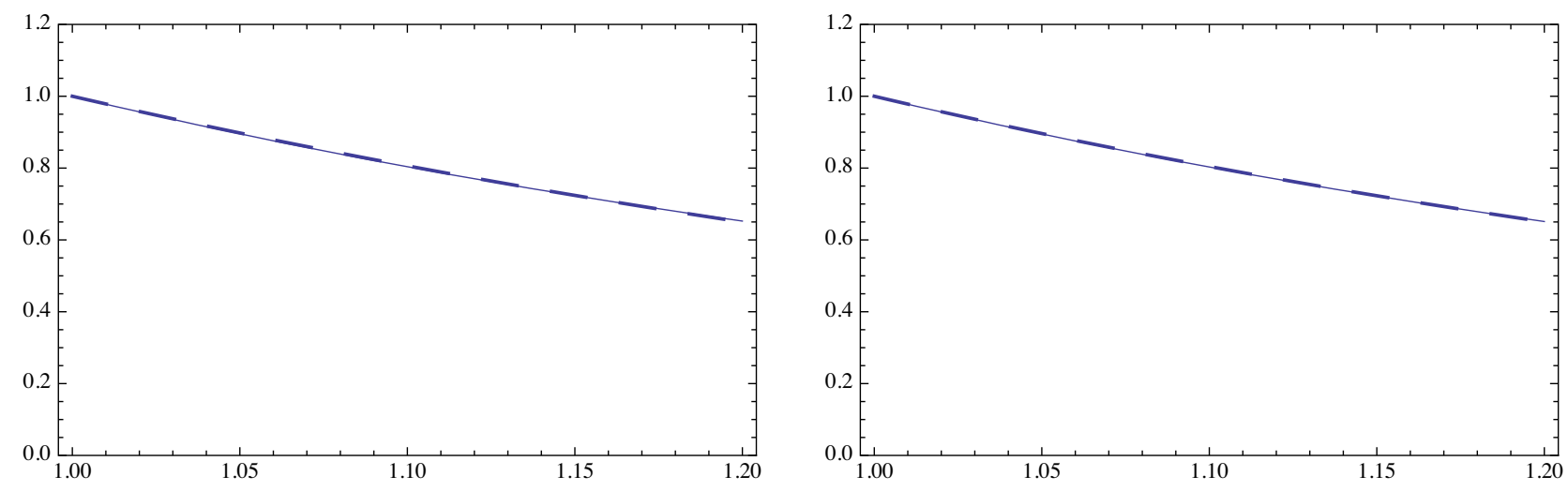

FIG. 2. Fits to the ratio $R_{2}(w) \simeq \xi_{\Lambda}(w)(8)$, (9) with the Isgur-Wise function (2) (continuous curve) using the parametrization of the lattice data up to the first order in the $z$ expansion (dashed curve, left) that yields the slope $\rho_{\Lambda}^{2} \simeq 2.24$, and up to the second order (dashed curve, right) that gives $\rho_{\Lambda}^{2} \simeq 2.25$.

We take these central values at face value, to see if for each form factor we find reasonable results for the set of parameters,

$$
\rho_{\Lambda}^{2}, \quad m_{c}, \quad m_{b}, \quad \bar{\Lambda}, \quad A^{\prime}(1),
$$

and how these parameters compare between the different form factors, i.e., how dispersed they are.

Since we have an independent estimate of the slope of the IW function (11), (12), we now fix

$$
\rho_{\Lambda}^{2} \simeq 2.15
$$

and we use FindFit to perform constrained fits for $m_{c}$ and $m_{b}$ and $\bar{\Lambda}$, assuming the following domains, from different analyses within HQET [35,36], in GeV units:

$$
1.15 \leq m_{c} \leq 1.35, \quad 4.10 \leq m_{b} \leq 4.40, \quad 0.60 \leq \bar{\Lambda} \leq 0.90,
$$

and the slope of the $1 / m_{Q}$ form factor $A^{\prime}(1)$ as a free parameter.

We take now the data for each form factor, the central values of Fig. 12 of [13], that are fitted by the first order $z$ expansion. Choosing a number of values of these $z$-expansion curves and the HQET model up to $O\left(1 / m_{Q}\right)$ of Appendix A, the resulting fit obtained with FindFit gives the plots of Fig. 3 and the parameters of Table 1.

We summarize the values for the parameters obtained from the fits for the different form factors in Table 1.

Let us comment on Fig. 3 and Table 1. First, the values obtained for the parameters $m_{c}, m_{b}$ and $\bar{\Lambda}$ are of course within the imposed limits (16). The fits are quite good for all form factors, except for $g_{+}\left(\Lambda_{b} \rightarrow \Lambda_{c}\right)$ and $g_{\perp}\left(\Lambda_{b} \rightarrow \Lambda_{c}\right)$ at large $q^{2}$ or near zero recoil $w=1$. In the lattice data, one sees that $g_{\perp}\left(q_{\max }^{2}\right), g_{+}\left(q_{\max }^{2}\right)<1$, while the calculation of the model gives $g_{\perp}\left(q_{\max }^{2}\right)=g_{+}\left(q_{\max }^{2}\right)=1$.
The discrepancy is due to the same $1 / m_{Q}^{2}$ correction at $w=1$, since one has

$$
g_{+}\left(q_{\max }^{2}\right)=g_{\perp}\left(q_{\max }^{2}\right) .
$$

Indeed, it is well-known that at zero recoil $w=1$ there is a negative $1 / m_{Q}^{2}$ correction for $g_{\perp}\left(q_{\max }^{2}\right)$, and this explains the discrepancy between the lattice data and the model. The $1 / m_{Q}^{2}$ correction satisfies a sum rule that gives this sign, see, for example, the discussion of the meson form factor $F_{D^{*}}$ at zero recoil in the review paper [37].

As a numerical example, from the range (13), we adopt for $\rho_{\Lambda}^{2} \simeq 2.15$, in Table 1 , the following ranges for the quark masses and subleading parameters:

$$
\begin{aligned}
m_{c} & \simeq 1.25 \pm 0.10 \mathrm{GeV}, \quad m_{b} \simeq 4.25 \pm 0.15 \mathrm{GeV} \\
\bar{\Lambda} & \simeq 0.75 \pm 0.15 \mathrm{GeV}, \quad A^{\prime}(1) \simeq-0.35 \pm 0.15 \mathrm{GeV} .
\end{aligned}
$$

It is worth to emphasize that the parameter $A^{\prime}(1)$ turns out to be negative and sizeable. This is a new result from the present analysis of form factors. Interestingly, the sign and magnitude is in qualitative agreement with the expression obtained in the nonrelativistic quark model, $A^{\prime}(1)=-\frac{\bar{\Lambda}}{2} \rho_{\Lambda}^{2}$ [formula (126) of Ref. [36] ].

\section{Correlation between $\rho_{\Lambda}^{2}$ and the slope parameter $\boldsymbol{A}^{\prime}(1)$}

There is a correlation between $\rho_{\Lambda}^{2}$ and the slope parameter $A^{\prime}(1)$. Indeed, taking the heavy quark limit in the expression of the form factors, except for $A^{\prime}(1)$, one finds, for all six form factors, for small $w-1$,

$F(w) \simeq 1+\left[\left(\frac{1}{2 m_{b}}+\frac{1}{2 m_{c}}\right) A^{\prime}(1)-\rho_{\Lambda}^{2}\right](w-1)+O\left(\frac{\bar{\Lambda}}{m_{Q}}\right)$, 

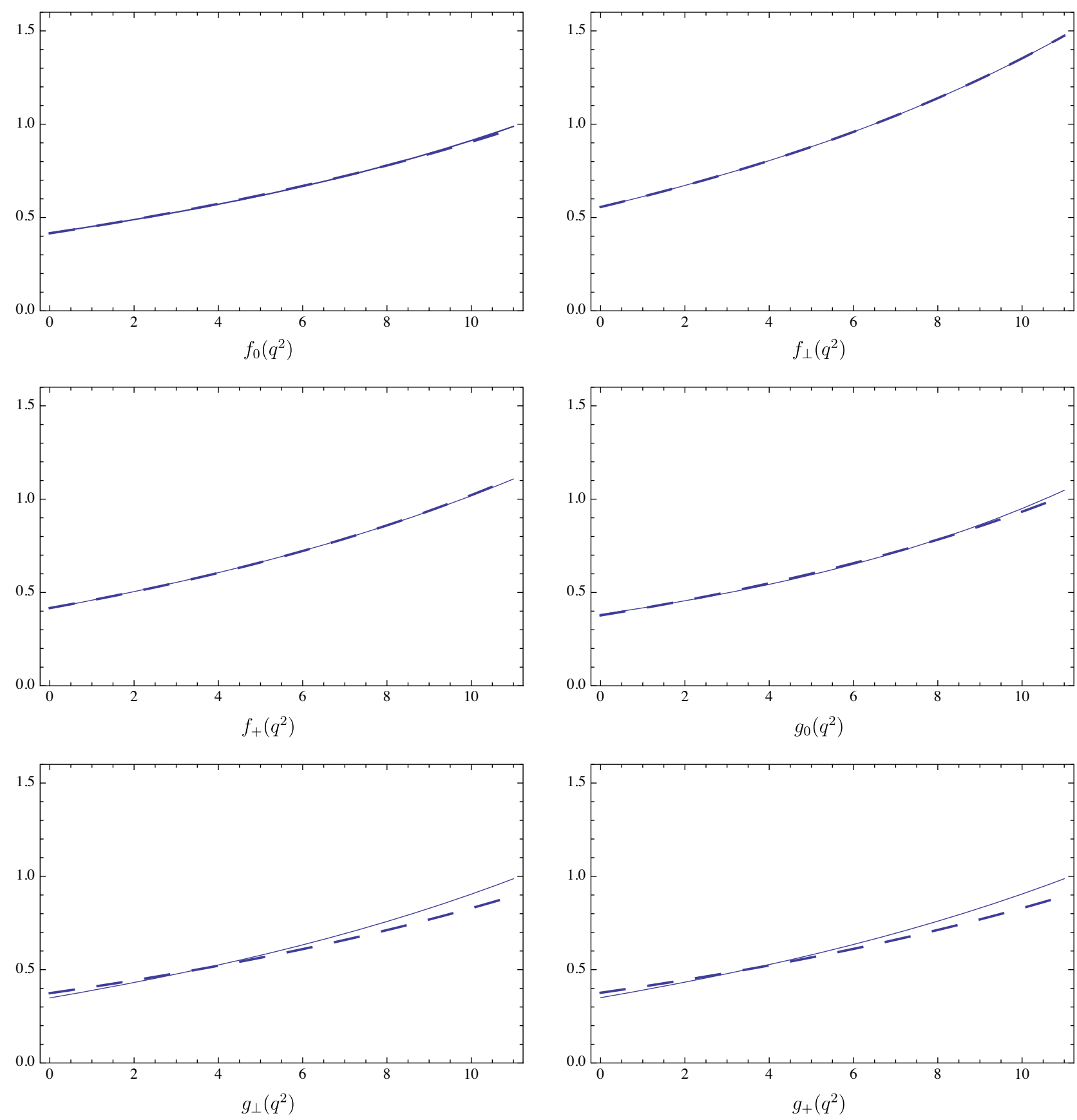

FIG. 3. Center values of lattice form factors in first order of the $z$ expansion [13] (dashed lines) compared to the fit using the HQET model up to $O\left(1 / m_{Q}\right)$ (continuous curves) obtained from FindFit.

that yields an effective slope,

$F^{\prime}(1) \simeq\left[\left(\frac{1}{2 m_{b}}+\frac{1}{2 m_{c}}\right) A^{\prime}(1)-\rho_{\Lambda}^{2}\right]+O\left(\frac{\bar{\Lambda}}{m_{Q}}\right)$.

Of course, one must take into account that the terms $O\left(\frac{\bar{\Lambda}}{m_{Q}}\right)$ contribute to the coefficient of $\rho_{\Lambda}^{2}$, so that the relation is somewhat different according to the form factors (Appendix A). For instance,

$f_{\perp}^{\prime}(1) \simeq\left(\frac{1}{2 m_{b}}+\frac{1}{2 m_{c}}\right) A^{\prime}(1)-\left[1+\bar{\Lambda}\left(\frac{1}{2 m_{b}}+\frac{1}{2 m_{c}}\right)\right] \rho_{\Lambda}^{2}$,

and different expressions depending on $\frac{\bar{\Lambda}}{2 m_{Q}} \rho_{\Lambda}^{2}$ for the derivatives of the other form factors, as, for example, 
TABLE I. Fits with the constraints (16) and arbitrary value for $A^{\prime}(1)$.

\begin{tabular}{lcccc}
\hline \hline Form factor & $m_{c}(\mathrm{GeV})$ & $m_{b}(\mathrm{GeV})$ & $\bar{\Lambda}(\mathrm{GeV})$ & $A^{\prime}(1)(\mathrm{GeV})$ \\
\hline$f_{0}\left(\Lambda_{b} \rightarrow \Lambda_{c}\right)$ & 1.33 & 4.34 & 0.66 & -0.21 \\
$f_{\perp}\left(\Lambda_{b} \rightarrow \Lambda_{c}\right)$ & 1.15 & 4.13 & 0.90 & -0.35 \\
$f_{+}\left(\Lambda_{b} \rightarrow \Lambda_{c}\right)$ & 1.15 & 4.40 & 0.90 & -0.25 \\
$g_{0}\left(\Lambda_{b} \rightarrow \Lambda_{c}\right)$ & 1.35 & 4.10 & 0.60 & -0.41 \\
$g_{\perp}\left(\Lambda_{b} \rightarrow \Lambda_{c}\right)$ & 1.35 & 4.38 & 0.60 & -0.49 \\
$g_{+}\left(\Lambda_{b} \rightarrow \Lambda_{c}\right)$ & 1.35 & 4.10 & 0.60 & -0.50 \\
\hline \hline
\end{tabular}

$$
\begin{aligned}
f_{+}^{\prime}(1) \simeq & \left(\frac{1}{2 m_{b}}+\frac{1}{2 m_{c}}\right) A^{\prime}(1) \\
& -\left[1+\bar{\Lambda}\left(\frac{1}{2 m_{b}}+\frac{1}{2 m_{c}}-\frac{2}{m_{b}+m_{c}}\right)\right] \rho_{\Lambda}^{2} .
\end{aligned}
$$

These $O\left(1 / m_{Q}\right)$ differences between the slopes of the different form factors allow us to determine separately $\rho_{\Lambda}^{2}$ and $A^{\prime}(1)$, but the tendency of the correlation remains the same. This correlation indicates that with some increase of $\rho_{\Lambda}^{2}$, it is possible from the above discussion, $A^{\prime}(1)$, which is found negative for $\rho_{\Lambda}^{2} \simeq 2.15$, that it should decrease in magnitude, or even change its sign.

The values of Table 1 correspond to the choice $\rho_{\Lambda}^{2}=2.15$, the central value of the domain (13). Had we adopted a higher value for the slope, then $A^{\prime}(1)$ would be negative but with a smaller absolute magnitude, and for $\rho_{\Lambda}^{2} \simeq 2.5-2.6$, there is a change of sign for $A^{\prime}(1)$, although this depends on the particular form factor.

\section{ELASTIC IW FUNCTION FOR THE $Q q q$ SYSTEM IN THE BAKAMJIAN-THOMAS QUARK MODEL}

For the ground state, we have the total wave function,

$$
\psi^{s \mu}=\frac{1}{\sqrt{3}} \sum_{P(231)} \varphi_{231}^{\Lambda_{Q}} \varphi_{231}^{s} \chi_{231}^{\prime \mu} .
$$

The flavor wave function writes $\varphi_{231}^{\Lambda_{Q}}=\frac{1}{\sqrt{2}}\left(d_{2} u_{3}-u_{2} d_{3}\right) Q_{1}$, the spin wave function $\chi_{231}^{\prime \prime}$ is antisymmetric in the 2,3 quarks and the full antisymmetry of the baryon wave function follows from the antisymmetry of the color singlet wave function.

For the simple case of the nonrelativistic harmonic oscillator, the ground state internal wave function (see, for example, Appendix A of Ref. [38]) writes

$\varphi\left(\left\{\mathbf{p}_{i}\right\}\right)=(2 \pi)^{3}\left(\frac{3 \sqrt{3} R_{\rho}^{3} R_{\lambda}^{3}}{\pi^{3}}\right)^{1 / 2} \exp \left(-\frac{\mathbf{p}_{\rho}^{2} R_{\rho}^{2}+\mathbf{p}_{\lambda}^{2} R_{\lambda}^{2}}{2}\right)$, where the relative momentum variables $\mathbf{p}_{\rho}$ and $\mathbf{p}_{\lambda}$ for $m_{2}=$ $m_{3}=m$ are defined by

$$
\mathbf{p}_{\rho}=\frac{1}{\sqrt{2}}\left(\mathbf{p}_{2}-\mathbf{p}_{3}\right), \quad \mathbf{p}_{\lambda}=\sqrt{\frac{3}{2}} \frac{m_{1}\left(\mathbf{p}_{2}+\mathbf{p}_{3}\right)-2 m \mathbf{p}_{1}}{m_{1}+2 m} .
$$

The wave function (24) is normalized according to

$$
\int \prod_{i=1}^{n} \frac{d \mathbf{p}_{i}}{(2 \pi)^{3}} \delta\left(\sum_{i=1}^{n} \mathbf{p}_{i}\right) \mid \varphi\left(\left.\left\{\mathbf{p}_{i}\right\}\right|^{2}=1,\right.
$$

or equivalently,

$$
\frac{1}{3 \sqrt{3}} \int \frac{d \mathbf{p}_{\rho}}{(2 \pi)^{3}} \frac{d \mathbf{p}_{\lambda}}{(2 \pi)^{3}} \mid \varphi\left(\left.\left\{\mathbf{p}_{\rho}, \mathbf{p}_{\lambda}\right\}\right|^{2}=1\right.
$$

Some words of caution concerning the wave function (24). First, this expression is valid in the limiting case of equal masses for the two light quarks [38], but, in general, crossed terms of the form $\mathbf{p}_{\rho} \cdot \mathbf{p}_{\lambda}$ could appear. Here, we restrict ourselves to the simplest case of the nonrelativistic harmonic oscillator with two light quarks of equal mass.

Assuming that the harmonic oscillator spring constant is flavor independent, the reduced radii $R_{\rho}$ and $R_{\lambda}$ are given, in terms of the equal mass baryon radius $R$, by the expressions,

$$
R_{\rho}^{4}=R^{4}, \quad R_{\lambda}^{4}=\frac{m_{1}+2 m}{3 m_{1}} R^{4}
$$

In the center-of-mass, $\mathbf{p}_{1}+\mathbf{p}_{2}+\mathbf{p}_{3}=0$, relations (25) give

$$
\mathbf{p}_{\rho}=\frac{1}{\sqrt{2}}\left(\mathbf{p}_{2}-\mathbf{p}_{3}\right), \quad \mathbf{p}_{\lambda}=\sqrt{\frac{3}{2}}\left(\mathbf{p}_{2}+\mathbf{p}_{3}\right)
$$

In the heavy quark limit $m \ll m_{1}$, the reduced radii (28) become

$$
R_{\rho}^{4}=R^{4}, \quad R_{\lambda}^{4}=\frac{R^{4}}{3} .
$$

Finally, we can obtain the explicit form of the baryon IW function with harmonic oscillator wave functions by replacing the expression (24) for the initial and final states in the general formula (B31) of Appendix B. 
We find

$$
\begin{aligned}
\xi_{\Lambda}\left(v \cdot v^{\prime}\right)= & (2 \pi)^{6} \frac{3 \sqrt{3} R_{\rho}^{3} R_{\lambda}^{3}}{\pi^{3}} \int \frac{d \mathbf{p}_{2}}{(2 \pi)^{3}} \frac{1}{p_{2}^{0}} \frac{d \mathbf{p}_{3}}{(2 \pi)^{3}} \frac{1}{p_{3}^{0}} \sqrt{\left(p_{2} \cdot v\right)\left(p_{3} \cdot v\right)\left(p_{2} \cdot v^{\prime}\right)\left(p_{3} \cdot v^{\prime}\right)} \\
& \times \exp \left\{-\left(\frac{3 R_{\lambda}^{2}+R_{\rho}^{2}}{4}\left[\left(p_{2} \cdot v^{\prime}\right)^{2}+\left(p_{3} \cdot v^{\prime}\right)^{2}-2 m^{2}\right]+\frac{3 R_{\lambda}^{2}-R_{\rho}^{2}}{4} 2\left[\left(p_{2} \cdot v^{\prime}\right)\left(p_{3} \cdot v^{\prime}\right)-\left(p_{2} \cdot p_{3}\right)\right]\right)\right\} \\
& \times \exp \left\{-\left(\frac{3 R_{\lambda}^{2}+R_{\rho}^{2}}{4}\left[\left(p_{2} \cdot v\right)^{2}+\left(p_{3} \cdot v\right)^{2}-2 m^{2}\right]+\frac{3 R_{\lambda}^{2}-R_{\rho}^{2}}{4} 2\left[\left(p_{2} \cdot v\right)\left(p_{3} \cdot v\right)-\left(p_{2} \cdot p_{3}\right)\right]\right)\right\} \\
& \times \frac{\left[m^{2}\left(1+v \cdot v^{\prime}\right)+m\left(v+v^{\prime}\right) \cdot\left(p_{2}+p_{3}\right)+\left(p_{2} \cdot v\right)\left(p_{3} \cdot v^{\prime}\right)+\left(p_{3} \cdot v\right)\left(p_{2} \cdot v^{\prime}\right)+\left(p_{2} \cdot p_{3}\right)\left(1-v \cdot v^{\prime}\right)\right]}{2 \sqrt{\left(p_{2} \cdot v+m\right)\left(p_{3} \cdot v+m\right)\left(p_{2} \cdot v^{\prime}+m\right)\left(p_{3} \cdot v^{\prime}+m\right)}},
\end{aligned}
$$

where the factor $\sqrt{\left(p_{2} \cdot v\right)\left(p_{3} \cdot v\right)\left(p_{2} \cdot v^{\prime}\right)\left(p_{3} \cdot v^{\prime}\right)}$ in the first line is due to the Jacobian of the change of variables in the BT scheme, and the complicated factor in the last line comes from the Wigner rotations [15], computed in the baryon case in Appendix B.

\section{A. An attempt to a concrete calculation of the IW function}

In Pervin et al. [20], the spectrum of heavy baryonic states has been studied with a linear + Coulomb Hamiltonian, with diagonalization in harmonic oscillator (HO) or pseudocoulombic (PC) bases and with kinetic energy either nonrelativistic or relativistic. In practice, the ground state wave function seems to be well represented by one Gaussian or one PC wave function. Choosing the Hamiltonian with relativistic energy and the $\mathrm{HO}$ basis, we read from their tables in the entry corresponding to the semi relativistic treatment in the basis of harmonic oscillator wave functions, the light quark mass and the ground state internal wave function necessary for the BT calculation, the latter being well approximated by one Gaussian, Eq. (24). They are given as follows:

$$
m=0.38 \mathrm{GeV}, \quad R_{\rho}^{2}=R_{\lambda}^{2}=2.16 \mathrm{GeV}^{-2} .
$$

We have computed the slope of the IW function (31) and have found

$$
\rho_{\Lambda}^{2}=4.01 .
$$

This value is much larger than the ranges (11), (12) determined in Sec. II.

Notice that the last factor in (31), due to the Wigner rotations, gives a very small numerical contribution to the slope.

It must be emphasized that this value is different and larger from the one given by the authors,

$$
\rho_{\Lambda}^{2}=1.33,
$$

the reason being that they use a nonrelativistic treatment to calculate the form factors, where, in principle,
$\rho_{\Lambda}^{2}=3 m^{2} R_{\lambda}^{2}$. This shows the tendency of the relativistic BT treatment to enhance the slope, which is what one would like. But, of course, the enhancement is too large, and it is worse with the PC basis.

On trying to understand this disappointing result, one notices that, as found by the authors of [20], there could be artifacts due to the smallness of the HO or PC expansion bases. On the other hand, in BT, there is for baryons, in contrast with mesons, a particular sensitivity of the value of $\rho_{\Lambda}^{2}$ to the detailed structure of the wave function, as we argue below. This emphasizes the need for larger bases.

\section{B. General discussion}

In the meantime, in view of this conclusion concerning the above Gaussian wave function, we proceed as follows. We pursue the investigation with the Gaussian shape (24) now considered as a model, with the objective of investigating the dependence of $\rho_{\Lambda}^{2}$ on the shape of the generic internal wave functions in the BT scheme, and in particular, to understand the high value obtained above, $\rho_{\Lambda}^{2} \simeq 4$. In fact, a somewhat similar discussion has been done numerically by Cardarelli and Simula [25] in the null plane formalism, which is known to be equivalent to the BT formalism in the heavy quark limit. However, one must avoid to give a physical interpretation to the Gaussian wave function, as we will see below. Here, we will rather develop a mathematical analysis to understand the variations of the slope $\rho_{\Lambda}^{2}$.

The formula (31) and its expansion at a small velocity to extract $\rho_{\Lambda}^{2}$, keeping for simplicity the terms coming from the Gaussians, and disregarding the contributions from the Jacobian and from the Wigner rotations gives

$$
\begin{aligned}
\rho_{\Lambda}^{2}= & 3 m^{2} R_{\lambda}^{2}+\frac{3 R_{\lambda}^{2}+R_{\rho}^{2}}{3}\left\langle{\overrightarrow{p_{2}}}^{2}+{\overrightarrow{p_{3}}}^{2}\right\rangle \\
& +\frac{3 R_{\lambda}^{2}-R_{\rho}^{2}}{2}\left\langle\frac{1}{3} \overrightarrow{p_{2}} \cdot \overrightarrow{p_{3}}+p_{2}^{0} p_{3}^{0}-m^{2}\right\rangle,
\end{aligned}
$$

with 2, 3 labeling the two light quarks, and $\langle\ldots\rangle$ denoting averages on the wave functions. The first term is the 
nonrelativistic contribution, the two others are relativistic corrections. The third term corresponds to crossed terms that, of course, are absent in mesons.

This formula shows that $\rho_{\Lambda}^{2}$ depends on two parameters, instead of one in the nonrelativistic limit (the term $\rho_{\Lambda}^{2}=3 m^{2} R_{\lambda}^{2}$ ), and one can get very high values because for $R_{\rho}^{2}<3 R_{\lambda}^{2}$ the last two terms in the expression are positive, and when $R_{\rho}^{2}$ becomes small, the $\langle\ldots\rangle$ averages become large. Indeed,

$$
\begin{gathered}
\left\langle{\overrightarrow{p_{2}}}^{2}\right\rangle=\left\langle{\overrightarrow{p_{3}}}^{2}\right\rangle=\frac{3}{4}\left(\frac{1}{R_{\rho}^{2}}+\frac{1}{3} \frac{1}{R_{\lambda}^{2}}\right) \\
\left\langle\overrightarrow{p_{2}} \cdot \vec{p}_{3}\right\rangle=\frac{3}{4}\left(-\frac{1}{R_{\rho}^{2}}+\frac{1}{3} \frac{1}{R_{\lambda}^{2}}\right) ;
\end{gathered}
$$

i.e., the momenta are equal, large, and antiparallel in average (and of course $\left\langle p_{2}^{0} p_{3}^{0}\right\rangle$ becomes also large). Then, the magnitude of $\rho_{\Lambda}^{2}$ is controlled by the ratio $R_{\lambda}^{2} / R_{\rho}^{2}$, and $\rho_{\Lambda}^{2}$ diverges when $R_{\rho}^{2} \rightarrow 0$ at fixed $R_{\lambda}$, in agreement with the numerical findings of Cardarelli and Simula in the null plane formalism [25].

Though the interpretation of this limit as corresponding to the pointlike diquark model given in this reference is at odds with our analysis of the quark-diquark model, which gives a small $\rho_{\Lambda}^{2}$, analogous to mesons (Sec. IV of the present paper), this is understandable, since in this limit $R_{\rho}^{2} \rightarrow 0$ the Gaussian is not the physical solution calculated from a QCD inspired Hamiltonian.

In fact, the last two terms in Eq. (35) diverge for $R_{\rho} / R_{\lambda} \rightarrow 0$ or $\infty$, but in one case, they have the same sign, and whence $\rho_{\Lambda}^{2}$ diverges, while in the other case, the divergences cancel when $R_{\lambda}$ is help fixed, and $\rho_{\Lambda}^{2}$ tends to a finite positive value.

It can be seen that these large values are related to the crossed term in the arguments of the two Gaussians with the coefficient $-2\left(\overrightarrow{p_{2}} \cdot \overrightarrow{p_{3}}\right)\left(3 R_{\lambda}^{2}-R_{\rho}^{2}\right)$ that give a large positive contribution balancing the factorizable one $-\left({\overrightarrow{p_{2}}}^{2}+{\overrightarrow{p_{3}}}^{2}\right)\left(3 R_{\lambda}^{2}+R_{\rho}^{2}\right)$ when $R_{\rho}^{2}$ approaches 0 .

On the other hand, imposing

$$
R_{\rho}^{2}=3 R_{\lambda}^{2}
$$

which corresponds to canceling the "crossed" terms, the expression (35) simplifies very much, and one finds

$$
\rho_{\Lambda}^{2}=3 m^{2} R_{\lambda}^{2}+2
$$

corresponding to the factorization of the wave function in $p_{2}, p_{3}$. The value (39) is not at odds with the slope determined from the lattice data in Sec. II, $\rho_{\Lambda}^{2} \simeq 2$.

To repeat, the BT result is quite unlike the nonrelativistic treatment, which always gives $\rho_{\Lambda}^{2}=3 m^{2} R_{\lambda}^{2}$, independently of $R_{\rho}^{2}$ : it depends now strongly on $R_{\rho} / R_{\lambda}$.
One sees that in the relativistic treatment $\rho_{\Lambda}^{2}$ can get arbitrary large values, while none of the two radii is supposed to be large.

Of course, let us recall that there is no claim to a dynamical calculation in all this discussion, but only an analysis of the relation between a generic Gaussian internal wave function and $\rho_{\Lambda}^{2}$, specific to the relativistic BT formalism.

However, it is interesting to note that the condition (39), which corresponds to a reasonable value of $\rho_{\Lambda}^{2}$, corresponds also to a situation where the distance between the two light quarks would be larger than the distance between each light quark and the heavy quark. This seems consistent with the intuition that the Compton wavelength of each light quark is large, and this is in fact the situation that seems to be found in dynamical calculations, like the one of Hernandez et al. in the nonrelativistic quark model [39], as well as in lattice studies [40,41]. Indeed, it is very important to recall that also in lattice QCD calculations one finds a $q q$ system with a large separation. Let us emphasize that in concluding these calculations, the term diquark must be taken with care since it is often meant on the contrary as a pointlike diquark, especially when speaking of diquark models. And, of course, these calculations question the very idea of a point-diquark model, at least when claiming to QCD inspired models, as we discuss in the next section.

Let us recall now another important conclusion coming from the above discussion. In the BT scheme, the value of the IW function slope for the $Q q q$ system depends strongly on the spatial configuration of the light diquark. This illustrates strikingly the contrast between the BT scheme and the nonrelativistic treatment of the center-of-mass motion of hadrons, for which there is no dependence of the slope on $R_{\rho}$, but only on $R_{\lambda}$. Therefore, in this relativistic scheme, there is a need to have a very good calculation of the wave function.

Interestingly, in Ref. [19], the wave function has been calculated very carefully, although in a spectroscopic model with nonrelativistic kinetic energy, which may be less worrying for a baryon. As to the authors themselves, they propose a rather low value $\rho_{\Lambda}^{2} \simeq 0.6-1$, , too low of course. But this value derives from the nonrelativistic treatment of the center-of-mass motion of the baryons.

It would be worth applying the BT formalism to the wave function of [19] to see whether it yields a correct slope. Indeed, the relativistic BT treatment could enlarge the value appreciably, as explained above.

\section{THE Q-POINTLIKE-DIQUARK MODELS}

As a possible alternative, the models with a pointlike diquark instead of two light quarks would be attractive because of their simplicity. One must note that the diquark may be also considered as extended, like in the works of Ebert et al. [26], but this is a different idea, outside of the present discussion (see also Ref. [42]). The quark-diquark model has been widely used to compute properties of the 
baryon spectrum and also relevant form factors in heavy baryon transitions [26].

Nevertheless, considering the several findings that have been recalled in the previous section, showing definitely that the $q q$ light quark subsystem has a large size, comparable with the one of the whole baryon, it is paradoxical to appeal to a pointlike diquark model. And indeed, our conclusion below in Sec. IV B is that such a model is not valid in the context of the QCD-inspired potentials, since it leads to a too low value $\rho_{\Lambda}^{2} \simeq 1$ as for mesons, which is quite logical since they are both two-body bound states with one heavy quark, and the potential is quite similar to the one for mesons.

On the other hand, this negative argument does not apply if we renounce to a QCD-inspired potential and introduce a nonstandard harmonic oscillator potential, whose strength can be freely adjusted. And indeed, we develop such a model as a provisory solution in the next section.

In Sec. IV A, we first develop the general BT framework for models with scalar pointlike diquark model, which will serve for both sections and then apply it to the model of Bing Chen et al., with a standard QCD-inspired potential, in Sec. IV B.

\section{A. Isgur-Wise functions in the BT scheme}

Let us indeed present the general calculation of the IW functions for a scalar $0^{+}$and $\overline{3}$ under color, pointlike particle, in the field of a heavy quark. As we will see now, there are no Wigner rotations in this case, and the BT results for IW functions simplify enormously.

\section{Elastic IW function}

One finds the simple expression (C3) of Appendix C,

$$
\begin{aligned}
\xi_{\Lambda}\left(v \cdot v^{\prime}\right)= & \int \frac{d \mathbf{p}_{2}}{(2 \pi)^{3}} \frac{1}{p_{2}^{0}} \varphi\left(\overrightarrow{\mathbf{B}_{v^{\prime}}^{-1} p_{2}}\right)^{*} \\
& \times \varphi\left(\overrightarrow{\mathbf{B}_{v}^{-1} p_{2}}\right) \sqrt{\left(p_{2} \cdot v\right)\left(p_{2} \cdot v^{\prime}\right)}
\end{aligned}
$$

with

$\varphi\left(\overrightarrow{\mathbf{B}_{v^{\prime}}^{-1} p_{2}}\right)^{*} \varphi\left(\overrightarrow{\mathbf{B}_{v}^{-1} p_{2}}\right)=\varphi\left(\left(p_{2} \cdot v^{\prime}\right)^{2}-m_{D}^{2}\right)^{*} \varphi\left(\left(p_{2} \cdot v\right)^{2}-m_{D}^{2}\right)$,

where $m_{D}$ denotes is the scalar diquark mass, of the order of twice the light quark mass, $m_{D} \simeq 2 m$.

\section{IW function for $L=1$ excited states}

In this case, one finds expression (C8) of Appendix C,

$$
\begin{aligned}
\sigma_{\Lambda}\left(v \cdot v^{\prime}\right)= & \frac{\sqrt{3}}{w^{2}-1} \int \frac{d \mathbf{p}_{2}}{(2 \pi)^{3}} \frac{1}{p_{2}^{0}} \varphi_{1}\left(\overrightarrow{\mathbf{B}_{v^{\prime}}^{-1} p_{2}}\right)^{*} \varphi\left(\overrightarrow{\mathbf{B}_{v}^{-1} p_{2}}\right) \\
& \times \frac{p_{2} \cdot\left(v-w v^{\prime}\right)}{\sqrt{\left(p_{2} \cdot v^{\prime}\right)^{2}-m_{D}^{2}}} \sqrt{\left(p_{2} \cdot v\right)\left(p_{2} \cdot v^{\prime}\right)},
\end{aligned}
$$

where one can see that the $1 /(w-1)$ singularity in the overall factor cancels with the numerator $p_{2} \cdot\left(v-w v^{\prime}\right)$ that vanishes when $w \rightarrow 1$.

\section{Bjorken sum rule}

From (40), the slope of the elastic IW function is

$$
\begin{aligned}
\rho_{\Lambda}^{2}= & -\xi_{\Lambda}^{\prime}(1) \\
& =-\frac{1}{24 \pi^{2}} \int_{0}^{\infty} d p \frac{1}{\sqrt{m_{D}^{2}+p^{2}}} p \varphi(p)^{*}\left\{\left(6 m_{D}^{2} p+5 p^{3}\right) \varphi(p)\right. \\
& \left.+4\left(m_{D}^{2}+p^{2}\right)\left[2\left(m_{D}^{2}+2 p^{2}\right) \varphi^{\prime}(p)+p\left(m_{D}^{2}+p^{2}\right) \varphi^{\prime \prime}(p)\right]\right\},
\end{aligned}
$$

and using (42) and the completeness relation for radial wave functions,

$$
\sum_{n} \varphi_{1}^{(n)}\left(p^{2}\right) \varphi_{1}^{(n) *}\left(k^{2}\right)=2 \pi^{2} \frac{\delta(p-k)}{p^{2}}
$$

we compute the sum $\sum_{n}\left|\sigma^{(n)}(1)\right|^{2}$ that gives

$$
\begin{aligned}
\sum_{n}\left|\sigma^{(n)}(1)\right|^{2}= & \frac{1}{24 \pi^{2}} \int_{0}^{\infty} d p \frac{1}{\sqrt{m_{D}^{2}+p^{2}}} \\
& \times p^{2}\left[p \varphi(p)^{*}+2\left(m_{D}^{2}+p^{2}\right) \varphi^{\prime *}(p)\right] \\
& \times\left[p \varphi(p)+2\left(m_{D}^{2}+p^{2}\right) \varphi^{\prime}(p)\right] .
\end{aligned}
$$

Integrating by parts this expression, one finds precisely the rhs of (43); i.e., one finds the Bjorken sum rule,

$$
\rho_{\Lambda}^{2}=\sum_{n}\left|\sigma^{(n)}(1)\right|^{2}
$$

Moreover, from the positivity of (45), one recovers the lower bound already established by heavy quark symmetry in Ref. [43],

$$
\rho_{\Lambda}^{2} \geq 0
$$

\section{An improved bound on the slope}

However, in this $Q$-diquark model, one can demonstrate a better lower bound due to the absence of the Wigner rotations, just by using the careful analysis of the different contributions to the meson IW slope given in Ref. [22].

One finds that the expression for the slope writes

$$
\rho_{\Lambda}^{2}=\frac{1}{2 \pi^{2}} \int_{0}^{\infty} d p p^{2} \varphi(p)^{*}\left(\frac{p^{0} z+z p^{0}}{2}\right)^{2} \varphi(p),
$$

where 


$$
p^{0}=\sqrt{p^{2}+m_{D}^{2}} \quad z=i \frac{d}{d p^{z}},
$$

and one has demonstrated that the lower bound of expression (48) is given by

$$
\rho_{\Lambda}^{2} \geq \frac{1}{3}
$$

i.e., an improved bound relative to the general bound (47).

Moreover, this bound was obtained on general grounds for a heavy baryon with light cloud $j=0$ [30] for the shape (2) of the IW function.

\section{B. Elastic and inelastic IW functions from wave functions in a QCD-inspired potential model}

The heavy baryon spectrum has been studied by Bing Chen et al. within the Q-diquark description with a QCD-inspired Hamiltonian [27],

$\left(\frac{\mathbf{p}^{2}}{2 \mu}-\frac{4 \alpha_{s}}{3 r}+b r+C+\right.$ spin dependent terms $) \psi=E \psi$,

where $\mu$ is the reduced mass,

$$
\mu=\frac{m_{D} m_{Q}}{m_{D}+m_{Q}},
$$

and $\mathbf{p}$ is the relative momentum of the heavy quark $Q$ and light pointlike diquark of mass $m_{D}$.

One notices that the potential in (51) is very similar to the one for a meson. This is easily understood: the interquark potential inside baryons is known to be half the one inside mesons, but on the other hand, there are two quarks on a diquark. This leads to a similarity in wave functions and finally for $\rho_{\Lambda}^{2}$, except that the mass here is heavier than for a light quark.

The wave functions corresponding to the spin-independent part of the Hamiltonian (51) are given in Appendix D for the heavy quark limit, and with the free $\beta$ parameter characterizing the variational basis chosen to $\beta=0.4$.

Inserting the heavy quark limit wave function $\varphi_{0}^{(n)}(\mathbf{p})$ (D3) into the expression (40) and with the reduced mass parameter that describes the spectrum within the Bing Chen et al. Hamiltonian,

$$
\mu=0.45 \mathrm{GeV},
$$

one finds the elastic IW function $\xi_{\Lambda}(w)$ of Fig. 4. An excellent fit is the "dipole" function (2),

$$
\xi_{\Lambda}(w)=\left(\frac{2}{w+1}\right)^{2 \rho_{\Lambda}^{2}}
$$

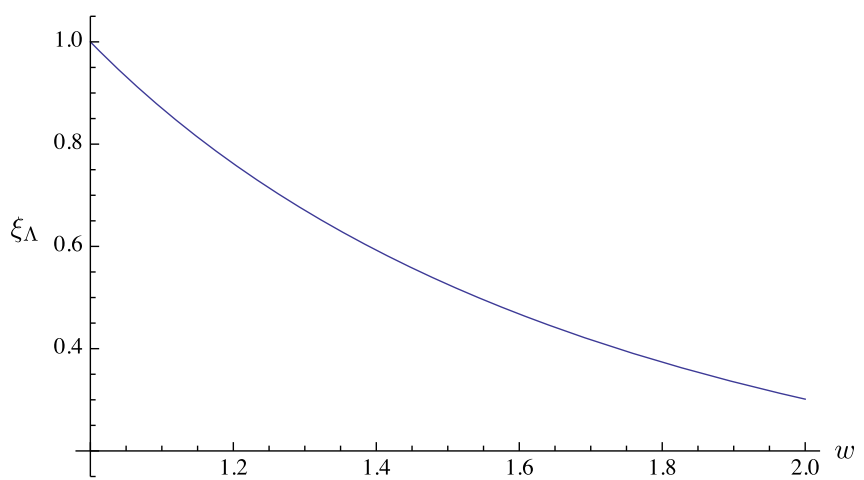

FIG. 4. Elastic Isgur-Wise function $\xi_{\Lambda}(w)$ obtained in the $Q$ diquark model with the Bing Chen et al. wave function (D3).

with the slope and curvature,

$$
\rho_{\Lambda}^{2}=1.27, \quad \sigma_{\Lambda}^{2}=2.25
$$

The slope is lower than the value $\rho_{\Lambda}^{2} \simeq 2$ obtained from the data of lattice QCD described in Sec. II. This low value is easily understood because this model amounts to a meson system, except for details of spin and for the light diquark mass which should be larger than for one quark. One must also take into account the difference of the definition for baryons against mesons that have $\mathrm{a}+1 / 4$ for the slope.

Consequently, the much too low value of $\rho_{\Lambda}^{2}$ (55) compels us to abandon the model, at least for form factors, as this is seen to be an unavoidable consequence of the scheme.

Nevertheless, we add for completeness the predictions of the model for the $L=1$ excitation. In the Bing Chen et al. model, from $\varphi_{0}^{(n)}(\mathbf{p})$ (D3) and $\varphi_{1}^{(n)}(\mathbf{p})$ (D4) and the parameter (53), we find the inelastic IW function $\sigma_{\Lambda}(w)$ of Fig. 5. The zero recoil value, the slope, and the curvature of the inelastic IW function are

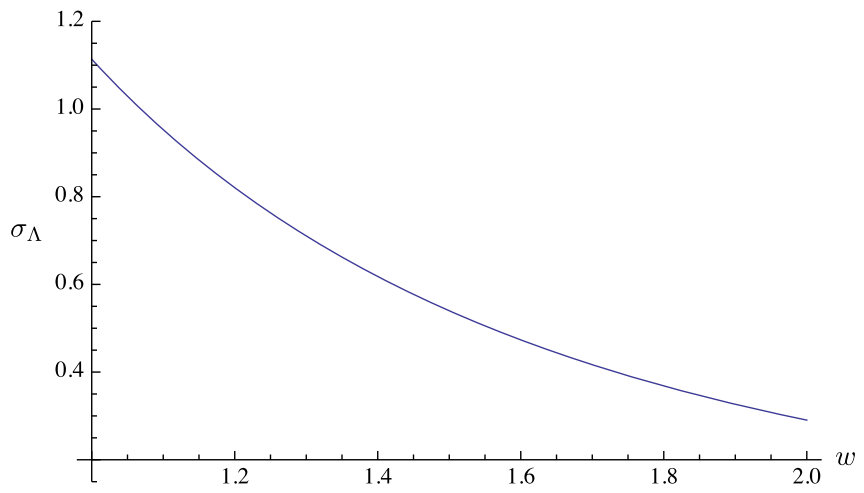

FIG. 5. Inelastic Isgur-Wise function $\sigma_{\Lambda}(w)$ obtained in the Q-diquark model with the Bing Chen et al. wave functions (D3), (D4). 


$$
\sigma_{\Lambda}(1)=1.11, \quad \sigma_{\Lambda}^{\prime}(1)=-1.89, \quad \sigma_{\Lambda}^{\prime \prime}(1)=4.16 .
$$

This corresponds to the lowest excitation $n=0$ of the inelastic $L=0 \rightarrow L=1$ IW function, and the Bjorken sum rule is almost saturated by it. Indeed, the rhs of (46) has a large contribution from the $n=0$ state,

$$
\rho_{\Lambda}^{2}=1.27 \geq\left[\sigma_{\Lambda}(1)\right]^{2}=1.11^{2}=1.23 .
$$

\section{SPECTRUM AND IW FUNCTIONS WITH HARMONIC OSCILLATOR WAVE FUNCTIONS}

As explained in the preceding Secs. III and IV, we have not obtained a satisfactory description of the baryon IW function neither using the internal wave function for three quarks deduced by Pervin et al. from a standard linear + Coulomb interquark potential nor using the two-body wave functions we have deduced from the pointlike diquark model of Bing Chen et al.

Compared to the lattice QCD result, $\rho_{\Lambda}^{2}$ has been found either much too large with a three-quark wave function of Pervin et al. or much too low for the diquark model of Bing Chen et al. This situation is quite different from the meson case, where the various standard spectroscopic models with relativistic kinetic energy combined with the BT scheme give consistently $\rho^{2} \simeq 1$, in good agreement with data.

Why one fails in the case of the Bing Chen et al. is clear from the discussion: the pointlike diquark assumption directly contradicts the dynamical calculations of the three quark system, in particular those of lattice QCD, which show definitely that the diquark system has a large extension. In fact, the model is close to a heavy meson, with a similar potential, and the BT formalism yields consistently $\rho_{\Lambda}^{2}$ not much above $\simeq 1$.

For the Pervin et al. wave function, one has no reason to suspect the linear + Coulomb spectroscopic Hamiltonian, and the reason is less obvious: the calculation of the wave function clearly requires larger bases, since the authors have observed a very large discrepancy between the $\mathrm{HO}$ and PC bases-all the more since in a three-quark system, as we have shown in Sec. III, the $\rho_{\Lambda}^{2}$ deduced from BT is very sensitive to the details of the wave functions, in contrast with a nonrelativistic treatment.

Then, leaving the correct solution of the three-quark case for a further investigation, we turn presently, for the phenomenological purpose of computing the observables, to a very simple model that is able to fit the observed $\rho_{\Lambda}^{2}$. It is a pointlike diquark model, but quite different from the one above with a QCD-inspired potential, with now a harmonic oscillator potential of arbitrary strength, which can be fitted both to the low-lying spectrum and to the desired $\rho_{\Lambda}^{2}$. Such a model is analogous in spirit with HO models used in the beginning of the quark model, except for the further simplification of using a pointlike diquark picture. The reason to expect sensible results from such a rough model is the fact that, in a first approximation, $\rho_{\Lambda}^{2}$ seems the main parameter controlling the heavy limit of the form factors, because dipole fits describe well the overall shape of $\xi_{\Lambda}(w)$ both in the model and on the lattice.

Let us assume harmonic-oscillator wave functions for the ground state $\Lambda_{Q}(Q=b, c)$ and for the tower of the radially excited $L=1$ states $(n \geq 0)$ according to the Hamiltonian,

$$
\left(\frac{\mathbf{p}^{2}}{2 \mu}+\frac{1}{2} K r^{2}+C\right) \psi=E \psi
$$

where the reduced mass $\mu$ is given by (52) and $m_{D}$ is the light diquark mass. The spring tension $K$ in Eq. (58) is flavor independent, the usual hypothesis for the harmonic oscillator Hamiltonian, and well satisfied for meson and baryon spectra.

From (58), in terms of the reduced mass $\mu$ and the $n=0$ level spacing,

$$
\omega=m_{\Lambda_{c}}\left(\frac{1-}{2}\right)-m_{\Lambda_{c}}\left(\frac{1+}{2}\right),
$$

the spring tension is given by

$$
K=\mu \omega^{2} .
$$

For very large $m_{Q}, \mu \simeq m_{D}$, where $m_{D}$ is the diquark mass $m_{D} \simeq 2 m$, and $m$ the light quark mass. For finite $m_{Q}$, one has $\mu<m_{D}$, and $\mu \simeq 0.4 \mathrm{GeV}$, in the case of charmed quarks.

According to (58), the wave functions read

$$
\varphi_{0}(\mathbf{p})=(4 \pi)^{3 / 4} R^{3 / 2} \exp \left(-\frac{\mathbf{p}^{2} R^{2}}{2}\right)
$$

$$
\begin{aligned}
\varphi_{1}^{(n)}(\mathbf{p})= & (-1)^{n}(4 \pi)^{3 / 4} 2^{n+1} \sqrt{\frac{n !(n+1) !}{(2 n+3) !}} R^{5 / 2}|\mathbf{p}| L_{n}^{3 / 2}\left(\mathbf{p}^{2} R^{2}\right) \\
& \times \exp \left(-\frac{\mathbf{p}^{2} R^{2}}{2}\right)
\end{aligned}
$$

that are normalized according to

$$
\int \frac{d \mathbf{p}}{(2 \pi)^{3}}|\varphi(\mathbf{p})|^{2}=1
$$

We will consider also the wave functions for $n>0$ and $L=1$ in order to verify that Bjorken SR holds.

Let us consider harmonic oscillator parameters that describe qualitatively the spectrum data, namely,

$m_{\Lambda_{c}}\left(\frac{1+}{2}\right)_{n=0}=2.286 \mathrm{GeV}, \quad m_{\Lambda_{c}}\left(\frac{1^{-}}{2}\right)_{n=0}=2.595 \mathrm{GeV}$, 
that gives the level spacing and reduced mass,

$$
\begin{aligned}
\omega & =m_{\Lambda_{c}}\left(\frac{1-}{2}\right)_{n=0}-m_{\Lambda_{c}}\left(\frac{1+}{2}\right)_{n=0} \simeq 0.309 \mathrm{GeV}, \\
\mu & =\frac{m_{D} m_{c}}{m_{D}+m_{c}} \simeq 0.40 \mathrm{GeV}
\end{aligned}
$$

for a light quark mass $m \simeq \frac{m_{D}}{2} \simeq 0.30 \mathrm{GeV}$ and a charm quark mass like in Sec. II, $m_{c} \simeq 1.25 \mathrm{GeV}$. Therefore, the spring tension (59) will be

$$
K=0.038 \mathrm{GeV}^{3} .
$$

Although the quantum numbers are still not confirmed, we consider now the natural candidate for the radial excitation, as assumed in [27],

$$
m_{\Lambda_{c}}\left(\frac{1+}{2}\right)_{n=1}=2.767 \mathrm{GeV}
$$

This gives the level spacing $m_{\Lambda_{c}}\left(\frac{1}{2}\right)_{n=1}-m_{\Lambda_{c}}\left(\frac{1}{2}{ }^{+}\right)_{n=0}=$ $0.481 \mathrm{GeV}$, while our simple model predicts

$m_{\Lambda_{c}}\left(\frac{1+}{2}\right)_{n=1}-m_{\Lambda_{c}}\left(\frac{1}{2}^{+}\right)_{n=0}=2 \omega=0.618 \mathrm{GeV}$,

some $20 \%$ higher.

However, since the IW function is defined in the heavy quark limit, we should take the reduced mass for $m_{Q} \rightarrow \infty$. To summarize, the spring tension $K$ is kept fixed, and the reduced mass becomes in the heavy quark limit $\mu \rightarrow m_{D}$, where $m_{D}$ is the diquark mass. One has then, in the heavy quark limit, the radius squared of the wave function,

$$
R^{2}=\frac{1}{\left(m_{D} K\right)^{1 / 2}} .
$$

One finds for some illustrative cases, for $m_{c}=1.25 \mathrm{GeV}$, the radius squared, the elastic slope $\rho_{\Lambda}^{2}$, and, using formula (42), the square of the $n=0$ inelastic IW function $L=0 \rightarrow L=1$ at zero recoil $\left|\sigma_{\Lambda}^{(0)}(1)\right|^{2}$,

$$
\begin{aligned}
m_{D} & =0.6 \mathrm{GeV}, \quad R^{2}=6.76 \mathrm{GeV}^{-2}, \\
\rho_{\Lambda}^{2} & =1.99, \quad\left|\sigma_{\Lambda}^{(0)}(1)\right|^{2}=1.93 \\
m_{D} & =0.8 \mathrm{GeV}, \quad R^{2}=5.34 \mathrm{GeV}^{-2}, \\
\rho_{\Lambda}^{2} & =2.48, \quad\left|\sigma_{\Lambda}^{(0)}(1)\right|^{2}=2.42 .
\end{aligned}
$$

Notice that within HQET one has, for baryons, the parameter $\bar{\Lambda}$,

$$
\bar{\Lambda} \simeq m_{D},
$$

and the value $\bar{\Lambda}=m_{D} \simeq 0.8 \mathrm{GeV}$ is precisely the one adopted in the HQET study of Leibovich and Stewart [35].

Here, to compute the interesting observables, we would like to adjust $m_{D}$ in order to obtain the central value for the slope obtained from the lattice data. We get roughly,

$$
\begin{aligned}
m_{D} & =0.67 \mathrm{GeV}, \quad R^{2}=6.20 \mathrm{GeV}^{-2}, \\
\rho_{\Lambda}^{2} & =2.15, \quad\left|\sigma_{\Lambda}^{(0)}(1)\right|^{2}=2.10 .
\end{aligned}
$$

We plot in Fig. 6 the elastic IW function for the set of parameters (72).

A very good fit to the IW function of Fig. 6 is given by the "dipole" form with $\rho_{\Lambda}^{2}=2.15$. Comparing the values for $\rho_{\Lambda}^{2}$ and $\left|\sigma_{\Lambda}^{(0)}(1)\right|^{2}$, we observe that the lowest radial excitation $n=0$ largely dominates the rhs of Bjorken SR (46).

Therefore, we conclude that the lowest inelastic IW function $(L=0, n=0) \rightarrow(L=1, n=0)$ is large, and thus, there is a good prospect for this transition to be well observed at $\mathrm{LHCb}$.

We plot in Fig. 7 the inelastic IW function $\sigma_{\Lambda}(w)$ with the set of parameters (72).

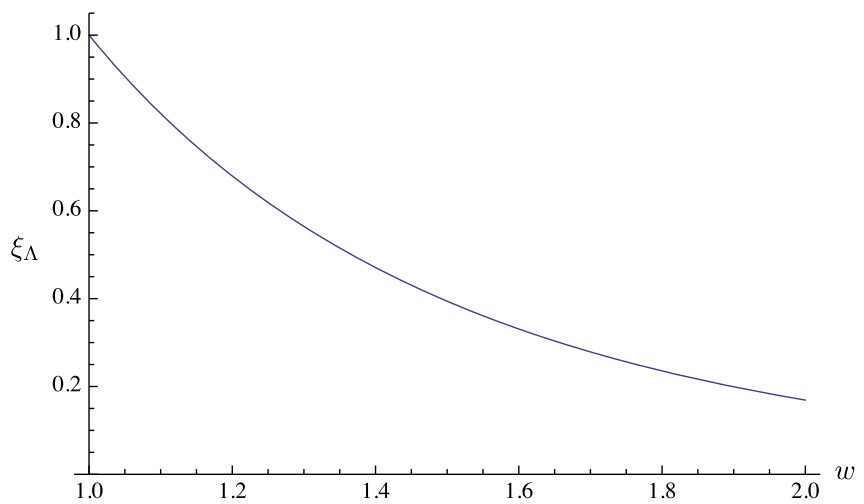

FIG. 6. Elastic Isgur-Wise function $\xi_{\Lambda}(w)$ obtained in the Q-diquark model with the harmonic oscillator wave function (61) and the parameters (72).

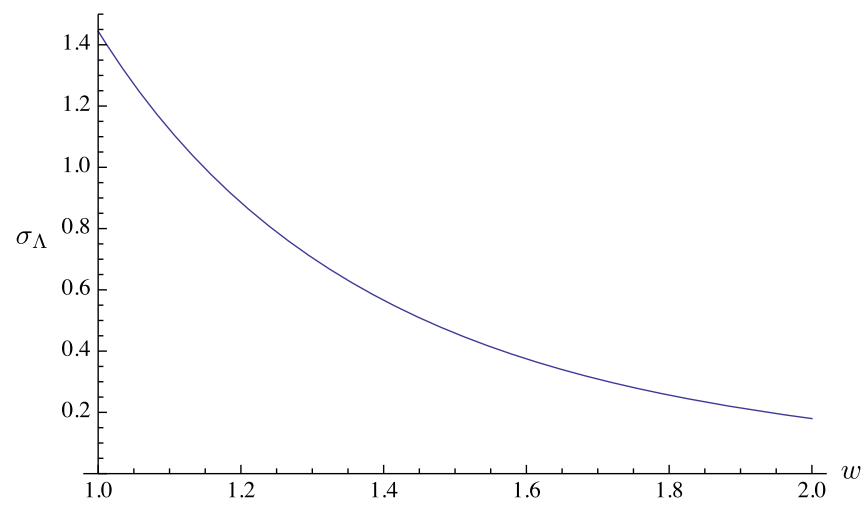

FIG. 7. Inelastic Isgur-Wise function $(L=0, n=0) \rightarrow(L=1$, $n=0)$ obtained in the Q-diquark model with the harmonic oscillator wave functions (61), (62) and the parameters (72). 
A reasonable "dipole" fit to Fig. 7 is given by

$$
\sigma_{\Lambda}(w)=\sigma_{\Lambda}(1)\left(\frac{2}{w+1}\right)^{2 \sigma_{\Lambda}^{\prime}(1)}
$$

with

$$
\sigma_{\Lambda}(1)=1.44, \quad \sigma_{\Lambda}^{\prime}(1)=2.57 .
$$

\section{OBSERVABLES IN $\Lambda_{b} \rightarrow \Lambda_{c}\left(\frac{1}{2}\right)^{ \pm} \ell \bar{\nu}$ TRANSITIONS}

The Mainz group has extensively formulated a number of different observables that could allow us to test the lepton flavor universality violation [44]. The expressions for the observables in terms of helicity amplitudes are given below in Appendix E.

\section{A. Observables for $\Lambda_{b} \rightarrow \Lambda_{c}\left(\frac{1}{2}^{+}\right) \ell \nu(\ell=e, \tau)$ transitions}

For the numerical calculations of the form factors and helicity amplitudes, we adopt the "dipole" shape expression for the IW function, with the slope (13) determined from the lattice data in Sec. II,

$$
\xi_{\Lambda}(w)=\left(\frac{2}{w+1}\right)^{2 \rho_{\Lambda}^{2}}, \quad \rho_{\Lambda}^{2}=2.15 \pm 0.10 .
$$

The ansatz (75) is close to the numerical calculation in the BT model within the Q-diquark scheme with HO NR internal wave function (62) with parameters (72).

For the function $A(w)$, we adopt

$$
A(w)=A^{\prime}(1)(w-1) f(w), \quad A^{\prime}(1)=-0.35 \pm 0.15,
$$

where the function $f(w)$ satisfies $f(1)=1$ and is introduced to soften the behavior of $A(w)$ for large $w$, near $w_{\max }$, because lattice data give only the slope (18). As an example, we could use simply $f(w)=\xi_{\Lambda}(w)$. So, we take

$A(w)=A^{\prime}(1)(w-1) \xi_{\Lambda}(w), \quad A^{\prime}(1)=-0.35 \pm 0.15$.

We will below comment further the role of the function $f(w)$ in (76), when discussing the comparison of the spectrum with experiment in Sec. VI A 1. Moreover, for $m_{c}, m_{b}$, we use the central values (18), and for $\bar{\Lambda}$, we adopt the value of our model (72), that agrees within errors with the lattice determination (18),

$m_{c}=1.25 \mathrm{GeV}, \quad m_{b}=4.25 \mathrm{GeV}, \quad \bar{\Lambda}=m_{D}=0.67 \mathrm{GeV}$.

The observables are given in Appendix E in terms of the helicity amplitudes $H_{\lambda_{2}, \lambda_{W}}^{V / A}$, that are given in terms of the form factors by the expressions,

$$
\begin{aligned}
H_{+\frac{1}{2} t}^{V / A} & =\frac{\sqrt{Q_{ \pm}}}{\sqrt{q^{2}}}\left(M_{\mp} f_{1}^{V / A} \pm q^{2} f_{3}^{V / A}\right) \\
H_{+\frac{1}{2} 0}^{V / A} & =\frac{\sqrt{Q_{\mp}}}{\sqrt{q^{2}}}\left(M_{ \pm} f_{1}^{V / A} \pm q^{2} f_{2}^{V / A}\right) \\
H_{+\frac{1}{2}+1}^{V / A} & =\sqrt{2 Q_{\mp}}\left(f_{1}^{V / A} \pm M_{ \pm} f_{2}^{V / A}\right) .
\end{aligned}
$$

In the physical processes, the $V-A$ chiral combination (E1) appears, and one has the parity relations between the $V / A$ helicity amplitudes,

$$
H_{-\lambda_{2},-\lambda_{W}}^{V}=H_{\lambda_{2}, \lambda_{W}}^{V}, \quad H_{-\lambda_{2},-\lambda_{W}}^{A}=-H_{\lambda_{2}, \lambda_{W}}^{A} .
$$

The form factors in (79) are described in Appendix A.1.

\section{The normalized theoretical rate compared to $\mathrm{LHCb}$ data}

Among the observables, only the shape of the LHCb data on the differential rate is known, but not the absolute magnitude [21]. We compare the LHCb rate normalized to one with the predictions of our model.

As a first remark, let us notice that our model of form factors up to $O\left(1 / m_{Q}\right)$ included, with essentially a single main parameter $\rho_{\Lambda}^{2}$, can well reproduce the LHCb normalized rate, as shown in Fig. 8. We have used the "dipolar" shape (75) with a slope slightly lower than the domain obtained from the lattice, $\rho_{\Lambda}^{2}=2$, the mass parameters (78), and the assumption $A(w)=0$.

We consider next the comparison with the parameters obtained from the lattice. Since the value of the slope $\rho_{\Lambda}^{2}=2$ is at the lower edge of the domain (75) and the assumption $A(w)=0$ is at odds with the values (76), we need to check the effect of the range of the lattice values.

We now compare the lattice parameters (75), (76), (78) with the LHCb data. With the aim of clarifying the discussion, we choose three sets of parametrizations, all of them with the mass parameters (78).

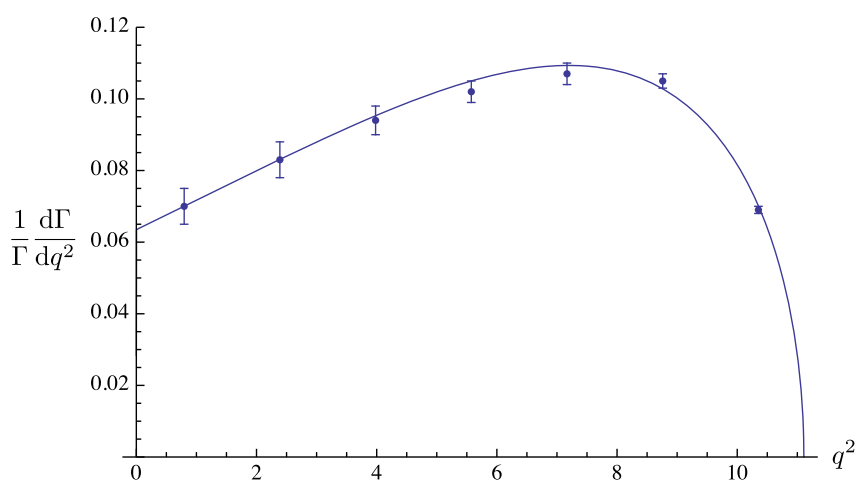

FIG. 8. Normalized rate $\frac{d \Gamma}{d q^{2}} / \Gamma$ compared to the LHCb data. We use the "dipolar" shape with $\rho_{\Lambda}^{2}=2$, the mass parameters (78), and we assume $A(w)=0$. 
(1) The lattice (75), (76) central values $\rho_{\Lambda}^{2}=2.15$, $\mathrm{A}^{\prime}(1)=-0.35$, and the linear approximation $A(w)=-0.35(w-1)$.

(2) The lattice (75), (76) central values $\rho_{\Lambda}^{2}=2.15$, $\mathrm{A}^{\prime}(1)=-0.35$, and softened $A(w)$ as $w$ increases, $A(w)=-0.35(w-1) \xi_{\Lambda}(w)$.

(3) The lattice (75), (76) smallest values $\rho_{\Lambda}^{2}=2.05$, $\mathrm{A}^{\prime}(1)=-0.20$, and softened $A(w)$ as $w$ increases, $A(w)=-0.20(w-1) \xi_{\Lambda}(w)$.

We compare these different choices to the data in Fig. 9. We observe that the set of parameters (1) describes the data very poorly, in particular due to the linear behavior of $A(w)$ and also due to a slightly too large slope. The set (2) is somewhat better, due to the softening of $A(w)$ at large $w$. Finally, the parameters (3) describe the data rather well, although not as well as the naive choice of Fig. 8.

The main conclusion of this discussion is that the $\mathrm{LHCb}$ normalized rate agrees within errors with the fit to the lattice data of form factors performed in Sec. II, that are summarized in formulas (75), (76), (78).

\section{Other observables}

We have seen that there are no sizeable differences between the set of parameters $\left(\rho_{\Lambda}^{2}, A^{\prime}(1)\right)=(2.05,-0.20)$ (Fig. 9) and the naive ansatz $\left(\rho_{\Lambda}^{2}, A^{\prime}(1)\right)=(2,0)$ (Fig. 8). For the calculation of the rest of the observables, we will use for simplicity the latter set. Moreover, all observables that are given by ratios of squared of helicity amplitudes are not sensitive to the small differences between the parameters used in Fig. 8 and the set (3) in Fig. 9.

\section{Comments on other observables$$
\Lambda_{b} \rightarrow \Lambda_{c}\left(\frac{1}{2}\right) \ell \nu(\ell=e, \tau)
$$

We compare the differential rate for $e$ and $\tau$ modes in Fig. 10.

It is interesting to observe the shape of the forwardbackward asymmetry in Fig. 11 for the electron mass and for the $\tau$ mass, where we observe a zero in the distribution.

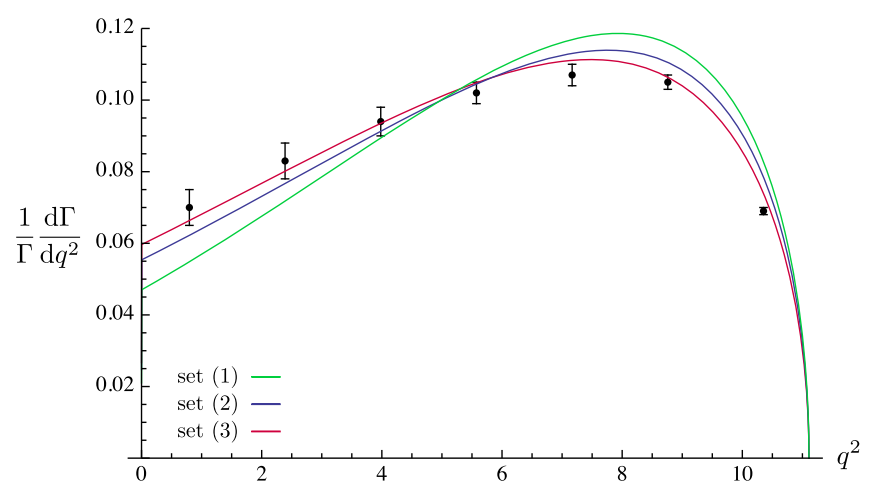

FIG. 9. Normalized rate $\frac{d \Gamma}{d q^{2}} / \Gamma$ compared to the $\mathrm{LHCb}$ data for the three sets of parameters (1), (2), and (3), respectively, lower, middle, and upper curves at $w=1$ (or $\left.q^{2}=0\right)$.

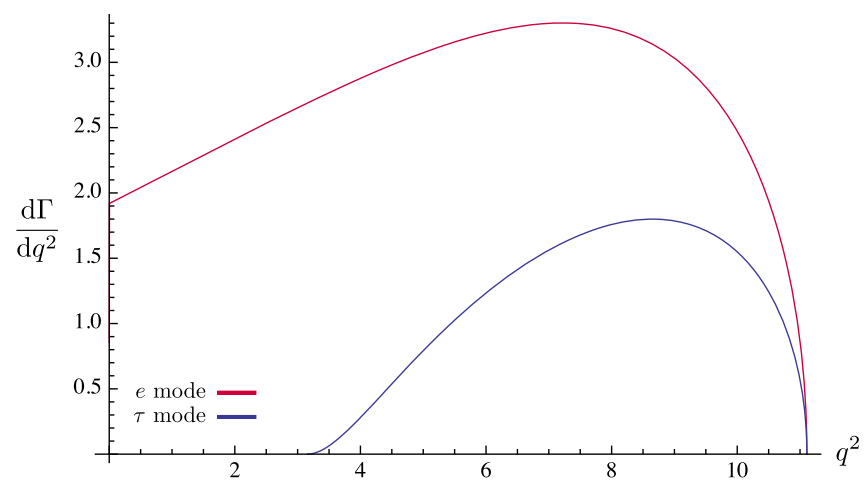

FIG. 10. $\frac{d \Gamma}{d q^{2}}$ for the electron and tau modes. In the electron case, one has $\frac{d \Gamma}{d q^{2}} \rightarrow 0$ for $q^{2} \rightarrow 0$.

From (E7), for $m_{\ell} \simeq 0$, the electron case, the FB asymmetry is given by

$$
A_{\mathrm{FB}}^{e}\left(q^{2}\right)=-\frac{3}{2} \frac{\mathcal{H}_{P}}{\mathcal{H}_{\mathrm{tot}}}=-\frac{3}{2} \frac{\left|H_{+\frac{1}{2}+1}\right|^{2}-\left|H_{-\frac{1}{2}-1}\right|^{2}}{\mathcal{H}_{\mathrm{tot}}}
$$

From the $V-A$ structure of the theory, the left-handed final baryon dominates, and therefore, we expect to have the inequality,

$$
\left|H_{+\frac{1}{2}+1}\right|^{2}<\left|H_{-\frac{1}{2}-1}\right|^{2}
$$

and similar inequalities for other helicity amplitudes. From (82) and (81), we expect $A_{\mathrm{FB}}^{e}\left(q^{2}\right)$ to be positive, as we observe in Fig. 11.

Let us see how the inequality (82) holds in our model of the form factors described in Appendix A.1. Just for illustration, keeping only the heavy quark limit terms, one finds indeed

$$
\begin{aligned}
\mathcal{H}_{P} & =\left|H_{+\frac{1}{2}+1}\right|^{2}-\left|H_{-\frac{1}{2}-1}\right|^{2} \\
& =-8 \sqrt{\lambda\left(m_{\Lambda_{b}}^{2}, m_{\Lambda_{b}}^{2}, q^{2}\right)}\left[\xi_{\Lambda}(w)\right]^{2}<0
\end{aligned}
$$

with $\lambda(a, b, c)=a^{4}+b^{4}+c^{4}-2 a^{2} b^{2}-2 b^{2} c^{2}-2 c^{2} a^{2}$, so that

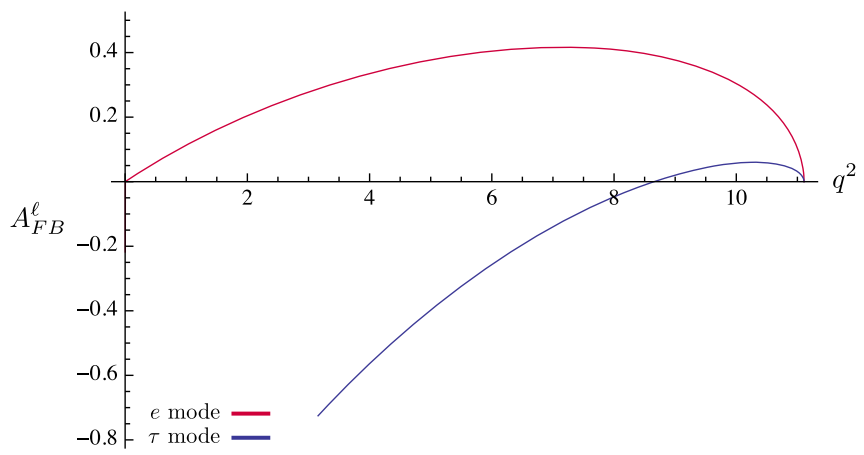

FIG. 11. Forward-backward asymmetry $A_{\mathrm{FB}}^{\ell}\left(q^{2}\right)$ for the electron and tau modes. 


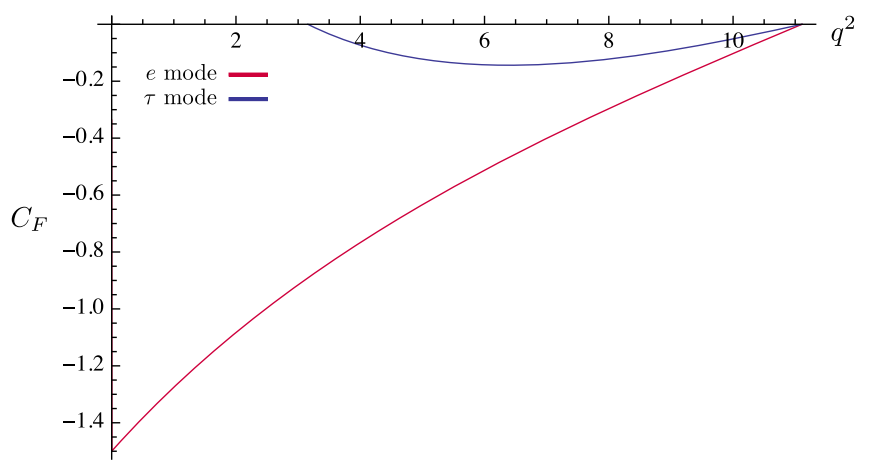

FIG. 12. Convexity parameter $C_{F}\left(q^{2}\right)$ for the electron and tau modes.

$$
\begin{aligned}
& q^{2}=m_{\Lambda_{b}}^{2}+m_{\Lambda_{c}}^{2}-2 m_{\Lambda_{b}} m_{\Lambda_{c}} w \\
& \lambda\left(m_{\Lambda_{b}}^{2}, m_{\Lambda_{c}}^{2}, q^{2}\right)=2 m_{\Lambda_{b}} m_{\Lambda_{c}} \sqrt{w^{2}-1}
\end{aligned}
$$

In the presence of a nonvanishing lepton mass $m_{\ell}$, the forward-backward $(F B)$ asymmetry (E7) presents a zero.

In particular, for the $\tau$ case, one has a zero in the $F B$ asymmetry as shown in Fig. 11. It is interesting to have a theoretical idea of the position of this zero, which, keeping only the heavy quark limit terms, is

$$
q_{0}^{2}\left(A_{\mathrm{FB}}^{\tau}\right)=m_{\tau} \sqrt{m_{b}^{2}-m_{c}^{2}}
$$

which qualitatively agrees with the one of Fig. 11, computed taking into account $1 / m_{Q}$ subleading terms.

The convexity parameter (E8) is plotted in Fig. 12 and the longitudinal and transverse hadron polarizations in Figs. 13 and 14.

Other observables, defined in Appendix E, are illustrated in Figs. 12-16, both for the massless and for the heavy lepton in the final state. In particular, we observe in Fig. 15 that in the $\tau$ case, the longitudinal lepton polarization $P_{z}^{\tau}\left(q^{2}\right)$ has a zero in the neighborhood of $q^{2} \simeq 4 . \mathrm{GeV}^{2}$. Indeed, performing an expansion in powers of $\frac{m_{\tau}}{m_{b}}$ and $\frac{m_{c}}{m_{b}}$, one finds the position of this zero,

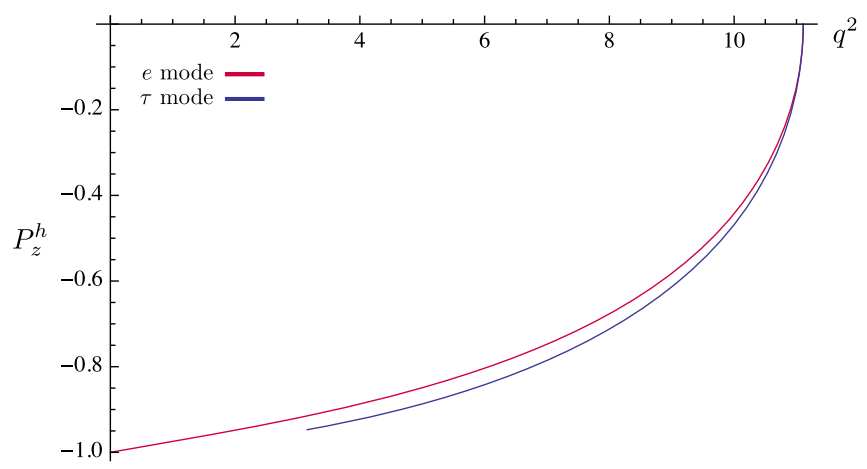

FIG. 13. Longitudinal hadron polarization $P_{z}^{h}\left(q^{2}\right)$ for the electron and tau modes.

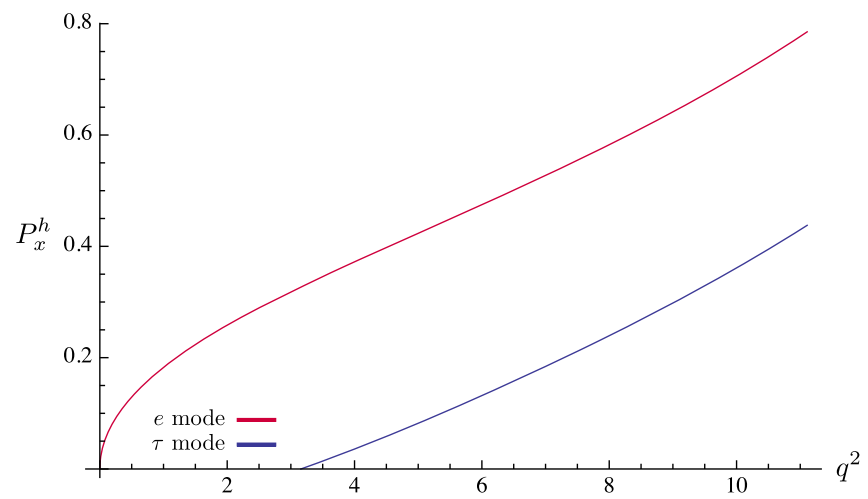

FIG. 14. Transverse hadron polarization $P_{x}^{h}\left(q^{2}\right)$ for the electron and tau modes.

$$
q_{0}^{2}\left(P_{z}^{\tau}\right) \simeq 2 m_{\tau}^{2}\left(1+2 \frac{m_{c}^{2}}{m_{b}^{2}}-3 \frac{m_{\tau}^{2}}{m_{b}^{2}}\right)
$$

that is numerically reasonable. The transverse lepton polarization is plotted in Fig. 16.

\section{B. Observables for $\Lambda_{b} \rightarrow \Lambda_{c}\left(\frac{1}{2}^{-}\right) \ell \nu(\ell=e, \tau)$ transitions}

To compute the form factors, we refer to the expressions and discussion of Appendix A.2, taken from Leibovich and Stewart [35]. We neglect the subleading Lagrangian perturbations (A14), that amounts to take [cf. (A16)] $\tilde{\sigma}(w) \equiv$ $\sigma_{\Lambda}(w)$ and the central value of (A20), $\hat{\sigma}_{1}=0$. We are left with the leading and subleading contributions proportional to the inelastic IW function.

For the inelastic IW function $\sigma(w) \equiv \sigma_{\Lambda}(w)$, we use the calculation (73), (74) done with the same parameters used in the elastic case (69),

$$
\sigma_{\Lambda}(w)=1.44\left(\frac{2}{w+1}\right)^{5.14} .
$$

Moreover, for the rest of the parameters, we also use the central values (78), and

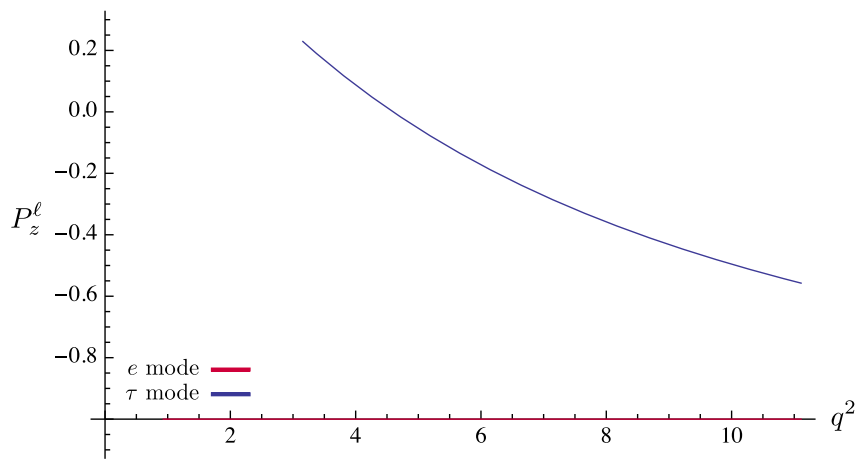

FIG. 15. Longitudinal lepton polarization $P_{z}^{\ell}\left(q^{2}\right)$ for the electron and tau modes. $P_{z}^{e}\left(q^{2}\right)$ is very close to -1 , unlike $P_{z}^{\tau}\left(q^{2}\right)$. 


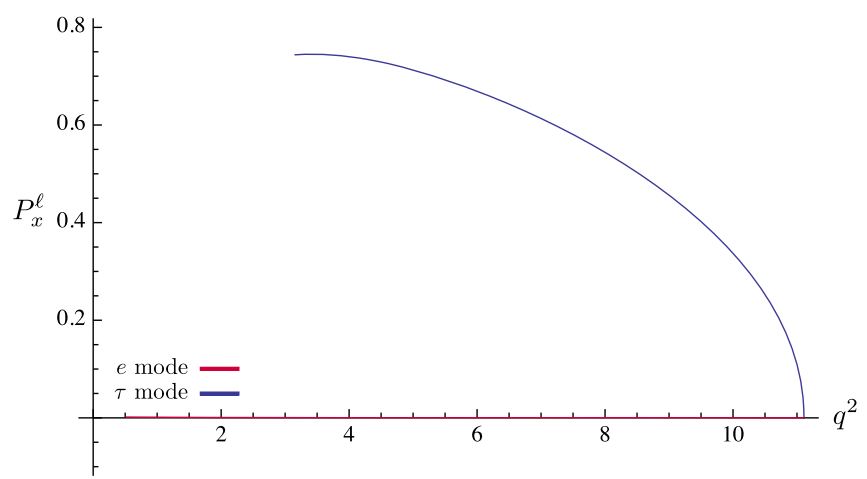

FIG. 16. Transverse lepton polarization $P_{x}^{\ell}\left(q^{2}\right)$ for the electron and tau modes. $P_{x}^{e}\left(q^{2}\right)$ is very small, unlike $P_{x}^{\tau}\left(q^{2}\right)$.

$$
\bar{\Lambda}^{\prime}=0.95 \mathrm{GeV} .
$$

The helicity amplitudes $H_{\lambda_{2}, \lambda_{W}}^{V / A}$ are in this case,

$$
\begin{aligned}
H_{+\frac{1}{2} t}^{V / A} & =\frac{\sqrt{Q_{\mp}}}{\sqrt{q^{2}}}\left(M_{ \pm} g_{1}^{V / A} \mp q^{2} g_{3}^{V / A}\right) \\
H_{+\frac{1}{2} 0}^{V / A} & =\frac{\sqrt{Q_{ \pm}}}{\sqrt{q^{2}}}\left(M_{\mp} g_{1}^{V / A} \mp q^{2} g_{2}^{V / A}\right) \\
H_{+\frac{1}{2}+1}^{V / A} & =\sqrt{2 Q_{ \pm}}\left(-g_{1}^{V / A} \pm M_{\mp} g_{2}^{V / A}\right) .
\end{aligned}
$$

In the physical processes, the $V-A$ chiral combination (E1) appears, and the parity relations between helicity amplitudes are now,

$$
H_{-\lambda_{2},-\lambda_{W}}^{V}=-H_{\lambda_{2}, \lambda_{W}}^{V}, \quad H_{-\lambda_{2},-\lambda_{W}}^{A}=H_{\lambda_{2}, \lambda_{W}}^{A} .
$$

The differential rate is plotted in Fig. 17 for the $e$ and $\tau$ modes.

For the electron case, the $F B$ asymmetry is given by (81) and again, although the final state parity has changed, from the $V-A$ structure of the theory, the left-handed final baryon dominates, and we expect to have the inequality (82) and $A_{\mathrm{FB}}^{e}\left(q^{2}\right)$ to be positive for all values of $q^{2}$, as we indeed observe in Fig. 18.

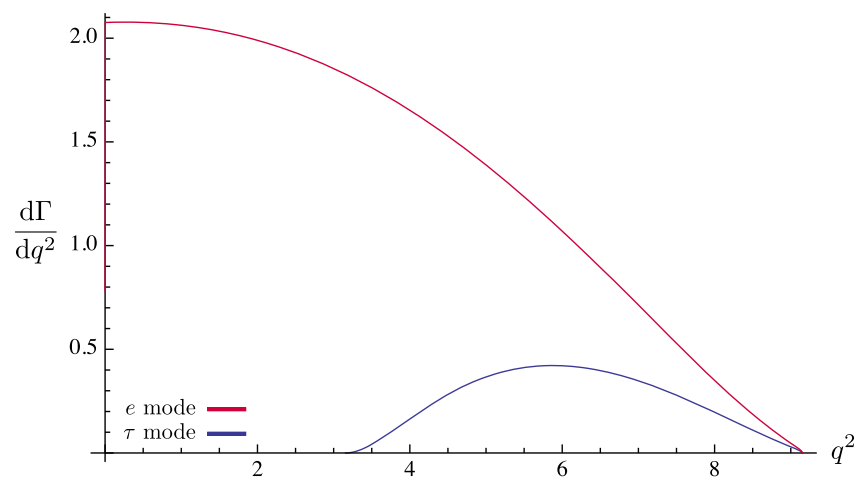

FIG. 17. $\frac{d \Gamma}{d q^{2}}$ for the electron and tau modes. In the electron case, one has $\frac{d \Gamma}{d q^{2}} \rightarrow 0$ for $q^{2} \rightarrow 0$.

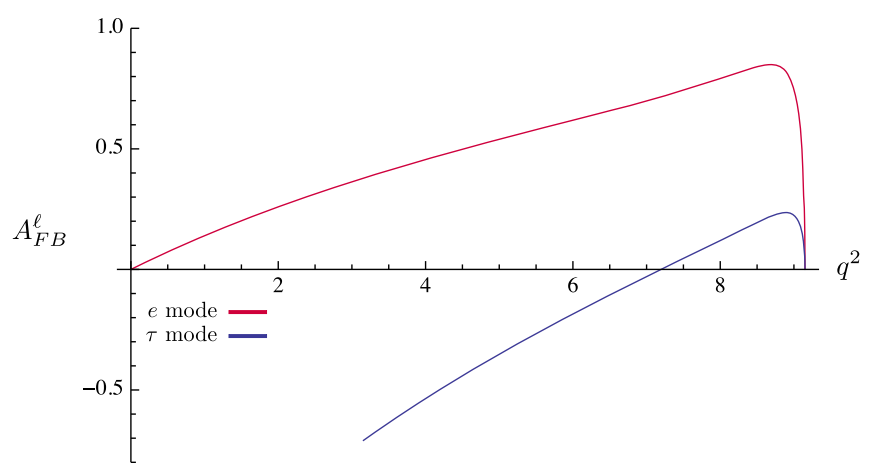

FIG. 18. Forward-backward asymmetry $A_{\mathrm{FB}}^{\ell}\left(q^{2}\right)$ for the electron and tau modes.

The convexity parameter, the longitudinal hardon polarization, the transverse hardon polarization, the longitudinal lepton polarization and the transverse lepton polarization are plotted, respectively, in Figs. 19-23.

It is interesting to see how the inequality (82) holds in our model of the form factors described in Appendix A.2. Similarly to what we have done above for the ground state, keeping only the heavy quark limit terms, one finds indeed

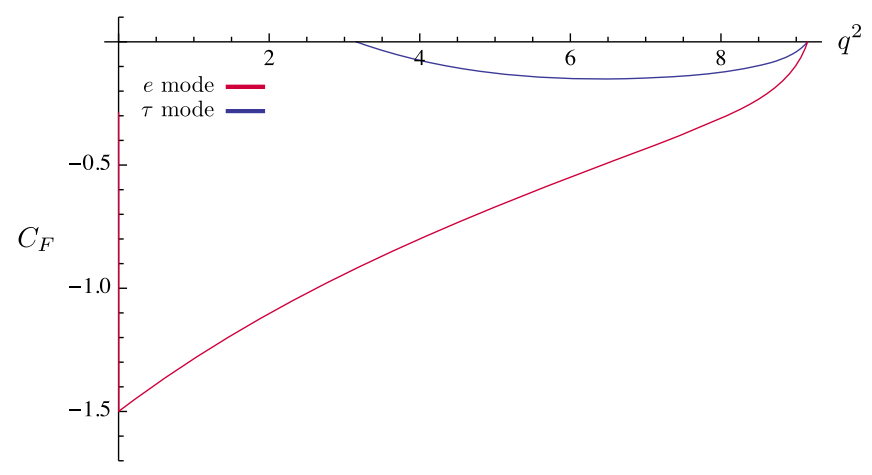

FIG. 19. Convexity parameter $C_{F}\left(q^{2}\right)$ for the electron and tau modes.

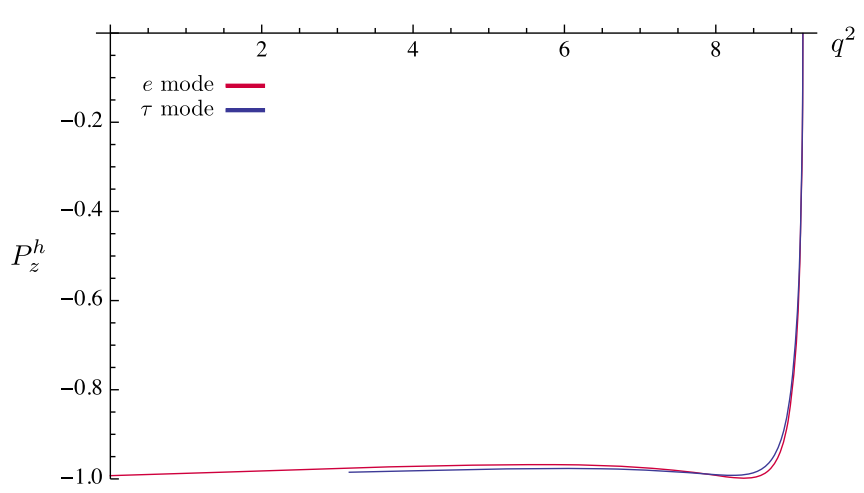

FIG. 20. Longitudinal hadron polarization $P_{z}^{h}\left(q^{2}\right)$ for the electron and tau modes. 


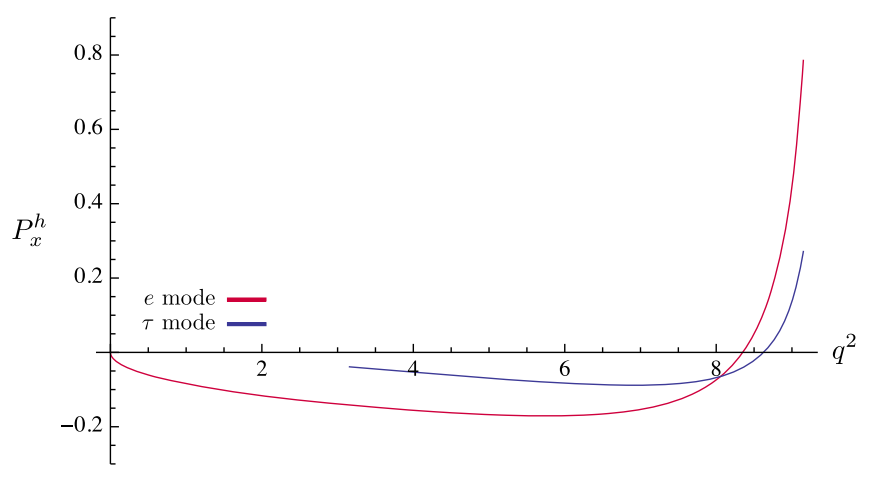

FIG. 21. Transverse hadron polarization $P_{x}^{h}\left(q^{2}\right)$ for the electron and tau modes.

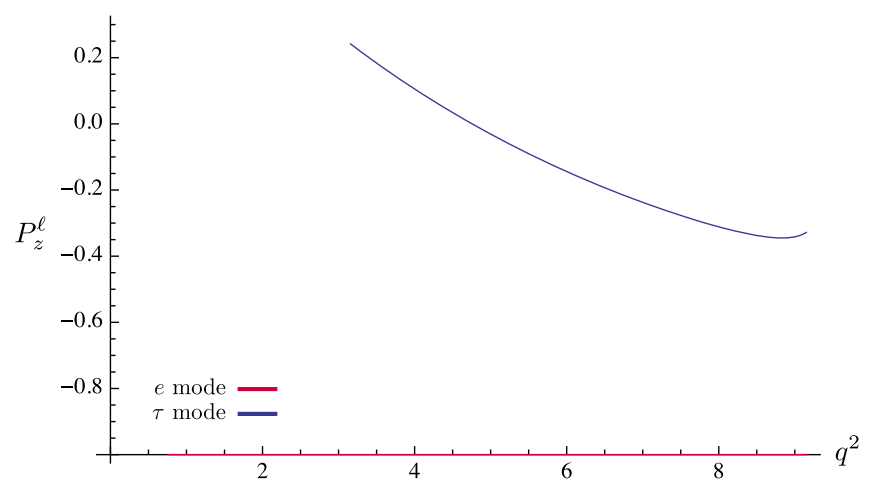

FIG. 22. Longitudinal lepton polarization $P_{z}^{\ell}\left(q^{2}\right)$ for the electron and tau modes. $P_{z}^{e}\left(q^{2}\right)$ is very close to -1 , unlike $P_{z}^{\tau}\left(q^{2}\right)$.

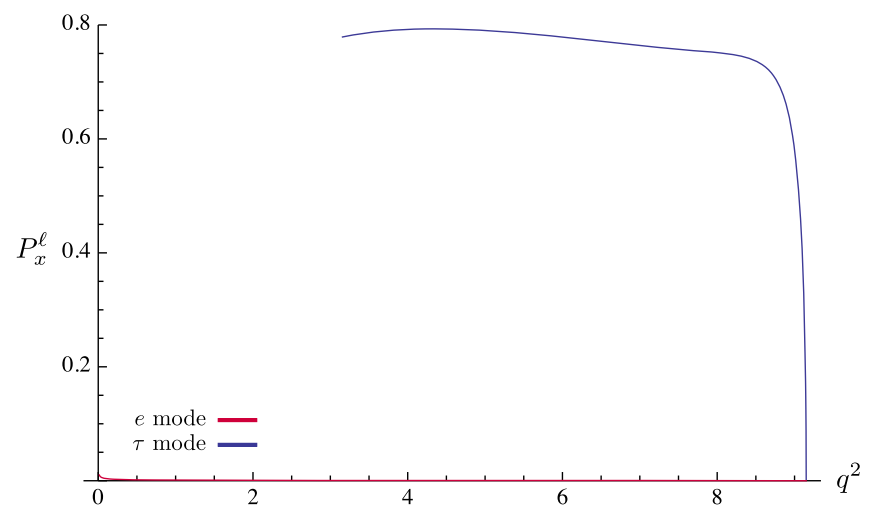

FIG. 23. Transverse lepton polarization $P_{x}^{\ell}\left(q^{2}\right)$ for the electron and tau modes. $P_{x}^{e}\left(q^{2}\right)$ is very small, unlike $P_{x}^{\tau}\left(q^{2}\right)$.

$$
\begin{aligned}
\mathcal{H}_{P} & =\left|H_{+\frac{1}{2}+1}\right|^{2}-\left|H_{-\frac{1}{2}-1}\right|^{2} \\
& =-\frac{16}{3} m_{b} m_{c}\left(w^{2}-1\right)^{3 / 2}\left[\sigma_{\Lambda}(w)\right]^{2}<0 .
\end{aligned}
$$

For the $\tau$ case, one has a zero in the $F B$ asymmetry. Keeping only the heavy quark limit terms, one finds the same value for the position of this zero as in the elastic case (85),

$$
q_{0}^{2}\left(A_{\mathrm{FB}}^{\tau}\right)=m_{\tau} \sqrt{m_{b}^{2}-m_{c}^{2}} .
$$

Also, we observe in Fig. 22 that, in the $\tau$ case, the longitudinal lepton polarization $P_{z}^{\tau}\left(q^{2}\right)$ has a zero in the neighborhood of $q^{2} \simeq 4 . \mathrm{GeV}^{2}$. However, in this case, unlike the ground state, we have not found a simple analytic expression for the position of this zero because the expansion in powers of $\frac{m_{\tau}^{2}}{m_{b}^{2}}$ and $\frac{m_{c}^{2}}{m_{b}^{2}}$ converges slowly.

\section{C. $\tau / \mathscr{\ell}$ observables sensitive to LFUV}

We now compute the relevant ratio of rates to test LFU (lepton flavor universality),

$$
R_{\tau / \ell}\left(\frac{1}{2}^{ \pm}\right)=\frac{\Gamma\left(\Lambda_{b} \rightarrow \Lambda_{c}\left(\frac{1}{2}^{ \pm}\right) \tau \nu\right)}{\Gamma\left(\Lambda_{b} \rightarrow \Lambda_{c}\left(\frac{1}{2}^{ \pm}\right) e \nu\right)}
$$

and we find, for the ground state,

$$
R_{\tau / \ell}\left(\frac{1+}{2}\right) \simeq 0.317
$$

and for the transition to the excited state,

$$
R_{\tau / \ell}\left(\frac{1}{2}^{-}\right) \simeq 0.141
$$

An interesting observable is the forward-backward asymmetry $A_{\mathrm{FB}}^{\ell}$, which has a very different behavior for the light leptons and for the $\tau$. In this latter case $A_{\mathrm{FB}}^{\tau}$, unlike $A_{\mathrm{FB}}^{e}$, presents a zero at $q_{0}^{2}\left(A_{\mathrm{FB}}^{\tau}\right) \simeq m_{\tau} \sqrt{m_{b}^{2}-m_{c}^{2}}$, for both $\frac{1}{2}^{+}$and $\frac{1}{2}^{-}$quantum numbers (Figs. 11,18 ). It would be very interesting to have a measurement of the position of the zero, that could be a test of the SM.

For both cases $\frac{1}{2}^{+}$and $\frac{1}{2}^{-}$(Figs. 13, 20), in the region of common phase space, the longitudinal hadron polarization is very similar for light leptons and for the $\tau$. This could be also an interesting test of the SM.

The transverse hadron polarization is very different for $\frac{1}{2}^{+}$and $\frac{1}{2}^{-}$(Figs. 14, 21). This shows clearly that this observable strongly depends on the internal wave function, as it is quite different for $\frac{1}{2}^{+}$and $\frac{1}{2}^{-}$states, that have very different wave functions.

For both cases $\frac{1}{2} \pm$ (Figs. 15, 22), the longitudinal electron polarization is very close to -1 , while it has a very different behavior for the $\tau$, that presents a zero at $q^{2} \simeq 4 \mathrm{GeV}^{2}$. These features could also provide interesting tests of the SM.

Also for both $\frac{1}{2}^{ \pm}$(Figs. 16, 23), the transverse electron polarization is very small while for the $\tau$ it is positive and sizeable for most of the phase space.

\section{Comparison with previous work}

\section{The work of Gutsche et al.}

Our calculation of the observables relies on the helicity formalism of the Mainz group paper by Gutsche et al. $[7,44]$, where the calculations for the ground state 
transitions $\Lambda_{b} \rightarrow \Lambda_{c}\left(\frac{1}{2}^{ \pm}\right) \ell \bar{\nu}$ for $\ell=e, \tau$ were done in their covariant confined quark model (CCQM). This work was recently extended to the $\Lambda_{b} \rightarrow \Lambda_{c}\left(\frac{1}{2}, \frac{3}{2}^{-}\right) \ell \bar{\nu}$ transitions for $\ell=e, \tau[28]$.

We would like to compare some aspects of their approach with our own.

First, in the CCQM, baryons are composites of $Q q q$, with the quantum numbers of the different states given by local interpolating fields with the correct quantum numbers, a compositeness condition, and a simple universal Gaussian form for the vertex functions. Moreover, the calculation is done at finite mass [45].

Our approach is a naive quark model in a Q-diquark scheme with a harmonic-oscillator potential, reproducing qualitatively the spectrum, and giving within the BT scheme a reasonable slope for the IW function, consistent with the lattice data. The quantum numbers for the $L=0$ and the $L=1$ states are then related by the Schrödinger equation giving the wave functions for both states, the main parameters being the $\mathrm{HO}$ radius and the masses.

It is encouraging that for the ground state transitions, $\Lambda_{b} \rightarrow \Lambda_{c}\left(\frac{1}{2}+\right) \ell \bar{\nu}(\ell=e, \tau)$, we find plots that are close to the ones of Ref. [7], in particular the position of the zero in the forward-backward asymmetry for the $\tau$ case. It would be very interesting to find the position of this zero in a theoretical scheme as model independent as possible, to put it on solid ground as a test of the Standard Model.

On the other hand, our numbers obtained for the ratios $R_{\tau / \ell}\left(\frac{1}{2}^{+}\right) \simeq 0.317$ and $R_{\tau / \ell}\left(\frac{1}{2}^{-}\right) \simeq 0.141$ are within the range of the predictions of Ref. [28], $R_{\tau / \ell}\left(\frac{1}{2}^{+}\right) \simeq 0.30 \pm 0.06$ and $R_{\tau / \ell}\left(\frac{1}{2}^{-}\right) \simeq 0.13 \pm 0.03$, so that they seem to be on a firm ground.

\section{Other HQET based analyses}

As pointed out above, we have adopted a simple HQET model, keeping the form factors up to first order in $1 / m_{Q}$ included, as given in the formulas of Appendix A, and then fitting the lattice results with the simple quark model within the BT scheme of Sec. V in order to compute the various observables in Sec. VI.

There are recent papers based on the HQET expansion that are relevant to our work, in particular, [46,47]. We will now comment in some detail the former of these papers.

The paper by Bernlochner et al. [46] relies on a HQET expansion in $1 / m_{Q}$ powers and radiative corrections, including $O\left(\Lambda_{\mathrm{QCD}} / m_{Q}\right)$ and $O\left(\alpha_{s} \Lambda_{\mathrm{QCD}} / m_{Q}\right)(Q=b, c)$ terms, as well as corrections of $O\left(\Lambda_{\mathrm{QCD}}^{2} / m_{c}^{2}\right)$.

Unlike Ref. [46], we do not take into account explicitly the QCD perturbative corrections to HQET, and therefore, it must be understood that our slope $\rho_{\Lambda}^{2}$ accounts by itself roughly for such effects.

Note that in the paper [46], the parameter $A^{\prime}(1)$ has been absorbed into the slope, introducing an effective slope, that in our notation reads $\rho_{\Lambda}^{2}-\left(\frac{1}{2 m_{b}}+\frac{1}{2 m_{c}}\right) A^{\prime}(1)$, dependent on the quark masses, and common to all form factors. This is of course correct, but only up to $O\left(\bar{\Lambda} \rho_{\Lambda}^{2} / m_{Q}\right)$ corrections, that differ according to the chosen form factor, as we have made explicit in two examples in Sec. II B 3. Then, fitting the slope $\rho_{\Lambda}^{2}$ and $A^{\prime}(1)$ from the different form factors allows us to separate both quantities. And therefore, it also follows that the slope of [46] $\zeta^{\prime}(1)$ differs from our $-\rho_{\Lambda}^{2}$.

We would like to distinguish our treatment of the lattice data and the one of [46]. The lattice data by Detmold et al., Ref. [13], have been parametrized in this paper by the $z$ expansion [35] for each form factor, with two fits, at $O(z)$ and $O\left(z^{2}\right)$.

Our aim has been a simple one, namely to compare our model for the form factors up to the first order in the $1 / m_{Q}$ expansion, with the two $O(z)$ and $O\left(z^{2}\right)$ fits done on the lattice data (our Figs. 1-3) by using the Mathematica package FindFit. We have chosen the following "synthetic data points" $q^{2}=0 ., 2 ., 4 ., 6 ., 8 ., 10 ., 11 ., q_{\max }^{2}$ from the lattice $z$ expansion fit. The agreement is good up to a couple of details that we underline, due to $1 / m_{Q}^{2}$ corrections. Although our fitting procedure is more naive of the one of [46], this paper only plots three points with rather large errors, at $q^{2}=0.5,5.5,10.0 \mathrm{GeV}^{2}$, compared to the range measured by the lattice $q^{2} / q_{\max }^{2}=[0.60-0.95]$ (Figs. 8-9 of [13]), that shows small errors.

On the other hand, although in Ref. [46] the slope $\zeta^{\prime}(1) \simeq 2.04$ is close to our determination, the curvature $\zeta^{\prime \prime}(1) \simeq 3.16$ (Table I) appears to be rather low in comparison with our fits. Indeed, with a $\rho_{\Lambda}^{2} \simeq 2.15$ with our dipole fit, which satisfies the HQET lower bound (5) on the curvature [29,30], $\sigma_{\Lambda}^{2}=\xi_{\Lambda}^{\prime \prime}(1) \geq \frac{3}{5}\left[\rho_{\Lambda}^{2}+\left(\rho_{\Lambda}^{2}\right)^{2}\right]$, we find $\sigma_{\Lambda}^{2} \simeq 5.7$, i.e., a term $+2.8(w-1)^{2}$ in the expansion. However, this is partly compensated by our next negative term $-3.0(w-1)^{3}$, which is still not negligible.

Finally, let us remark that [46] finds $R\left(\Lambda_{c}\right)=$ $0.324 \pm 0.004$, that is close to our ratio (94). Their Fig. 1 for $\frac{1}{\Gamma} \frac{d \Gamma}{d q^{2}}$ agrees within errors with our Fig. 8 and with our red curve of Fig. 9. This seems to show that the normalized decay rate is essentially given by the lattice data on the shape of the form factors, since both our work and [46] rely on fits to these data.

\section{CONCLUSIONS}

Our objective has been the calculation of the observables in the decays $\Lambda_{b} \rightarrow \Lambda_{c}^{ \pm} \ell \bar{\nu}$, that could provide tests of lepton flavor universality violation.

We have done this in a quark model that, unlike present lattice QCD calculations, allows not only the computation of transitions within the ground state, but also those to the $L=1$ excitations.

The BT method is very suited for such a calculation; the wave functions are three-dimensional, but the result is covariant in the heavy quark limit. 
The BT approach can explain the value of the slope of the baryon IW function $\rho_{\Lambda}^{2}$, very different from the nonrelativistic value, as it happens for mesons.

The slope $\rho_{\Lambda}^{2}$ is a very important parameter to describe the form factors, and it is at the same time a discriminant of the different models. The adopted dipolar fit for the IW function satisfies a number of theorems that have been found in the heavy quark limit of HQET that the different derivatives of the IW function must satisfy.

To test the different models, we have analyzed the lattice data on the form factors, and we have shown that the slope of the IW function is of the $\operatorname{order} \rho_{\Lambda}^{2} \simeq 2$. On the other hand, we have demonstrated that the slope of the subleading form factor $A^{\prime}(1)$ can be determined independently of $\rho_{\Lambda}^{2}$.

We have found a number of difficulties of the standard QCD-inspired Hamiltonian approaches, both in the three quark $Q q q$ model and also in quark pointlike-diquark models. We have thoroughly discussed these problems.

In particular, for the $Q q q$ system, we have discussed in detail the problems that we have found to describe the slope of the IW function $\rho_{\Lambda}^{2}$. As a model, we have adopted harmonic oscillator wave functions and studied the different limits according to the values of the radii $R_{\rho}^{2}, R_{\lambda}^{2}$ that correspond to the two Jacobi variables. This discussion gives an insight on the structure of the $Q q q$ system, in particular of the light $q q$ cloud, that can be helpful in the construction of realistic models for the $Q q q$ wave function.

In view of the encountered difficulties, we have adopted a preliminary quark pointlike-diquark model that allows a qualitative description of the spectrum, and of the IW slope $\rho_{\Lambda}^{2} \simeq 2$, in agreement with lattice data.

We have computed the different observables proposed by the Mainz group for both transitions $\Lambda_{b} \rightarrow \Lambda_{c}\left(\frac{1}{2}^{ \pm}\right) \ell \bar{\nu}$, emphasizing the differences between $e$ and $\tau$ transitions.
Using the Bjorken sum rule, we find in our model that the lowest inelastic IW function $\sigma_{\Lambda}(w)$, which describes the $(L=0) \rightarrow(L=1)$ transitions, is large, and thus, there is a good prospect for the decays $\Lambda_{b} \rightarrow \Lambda_{c}\left(\frac{1}{2}^{-}, \frac{3}{2}^{-}\right) \ell \bar{\nu}$ to be studied in detail at LHCb. Both decays depend on $\sigma_{\Lambda}(w)$ because the states $\frac{1}{2}^{-}, \frac{3}{2}^{-}$belong to the same doublet in the heavy quark limit.

We have seen that some observables, the forwardbackward asymmetries, and the longitudinal lepton polarization present a zero at some characteristic value of $q^{2}$ for the $\tau$ case. The positions of these zeros could provide tests of physics beyond the Standard Model. In particular, the forward-backward asymmetry for both $\Lambda_{b} \rightarrow \Lambda_{c}\left(\frac{1}{2}{ }^{ \pm}\right) \tau \nu$ cases presents a zero for $q^{2} \simeq m_{\tau} \sqrt{m_{b}^{2}-m_{c}^{2}}$.

We do not study for the moment the case of the inelastic transitions $\Lambda_{b} \rightarrow \Lambda_{c}\left(\frac{3}{2}^{-}\right) \ell \bar{\nu}(\ell=e, \tau)$. In the quark model, one has to consider a $S=1$ diquark coupled to $L=1$. We postpone this study since we would like to analyze and compute the different observables that could be interesting for our purpose, besides the one computed in Ref. [28].

As a word of caution, we have to say that our results for the observables are preliminary, as we will need in the future to treat systematically the three quark system $Q q q$ to study the spectrum and the IW function, and then turn to phenomenological applications.

\section{ACKNOWLEDGMENTS}

We are indebted to Misha Ivanov, Jurgen Koerner, and Valery Lyubovitskij for enlightening correspondence on their work on $\Lambda_{b}$ decays.

\section{APPENDIX A: BARYON FORM FACTORS AND THE HEAVY QUARK EXPANSION}

\section{Baryon form factors $\Lambda_{b} \rightarrow \Lambda_{c}\left(\frac{1}{2}^{+}\right)$up to order $1 / m_{Q}$}

From Falk and Neubert [48], the six conventional form factors $f_{i}, g_{i}(i=1,2,3)$ write

$$
\begin{aligned}
\left\langle\Lambda_{c}\left(p^{\prime}, s^{\prime}\right)\left|\bar{c} \gamma^{\mu} b\right| \Lambda_{b}(p, s)\right\rangle & =\bar{u}_{\Lambda_{c}}\left(p^{\prime}, s^{\prime}\right)\left[f_{1} \gamma^{\mu}-i f_{2} \sigma^{\mu \nu} q_{\nu}+f_{3} q^{\mu}\right] u_{\Lambda_{b}}(p, s) \\
\left\langle\Lambda_{c}\left(p^{\prime}, s^{\prime}\right)\left|\bar{c} \gamma^{\mu} \gamma_{5} b\right| \Lambda_{b}(p, s)\right\rangle & =\bar{u}_{\Lambda_{c}}\left(p^{\prime}, s^{\prime}\right)\left[g_{1} \gamma^{\mu}-i g_{2} \sigma^{\mu \nu} q_{\nu}+g_{3} q^{\mu}\right] \gamma_{5} u_{\Lambda_{b}}(p, s) .
\end{aligned}
$$

The form factors $f_{3}, g_{3}$ will contribute to $\Lambda_{b} \rightarrow \Lambda_{c} \tau \nu$.

The alternative notation, convenient for HQET, is given in terms of the four velocities,

$$
\begin{gathered}
\left\langle\Lambda_{c}\left(v^{\prime}, s^{\prime}\right)\left|\bar{c} \gamma^{\mu} b\right| \Lambda_{b}(v, s)\right\rangle=\bar{u}_{\Lambda_{c}}\left(p^{\prime}, s^{\prime}\right)\left[F_{1} \gamma^{\mu}+F_{2} v^{\mu}+F_{3} v^{\prime \mu}\right] u_{\Lambda_{b}}(v, s) \\
\left\langle\Lambda_{c}\left(v^{\prime}, s^{\prime}\right)\left|\bar{c} \gamma^{\mu} b \gamma_{5}\right| \Lambda_{b}(v, s)\right\rangle=\bar{u}_{\Lambda_{c}}\left(v^{\prime}, s^{\prime}\right)\left[G_{1} \gamma^{\mu}+G_{2} v^{\mu}+G_{3} v^{\prime \mu}\right] \gamma_{5} u_{\Lambda_{b}}(v, s) .
\end{gathered}
$$

The form factors $f_{i}, g_{i}$ write, in terms of the $F_{i}, G_{i}$, 


$$
\begin{aligned}
& f_{1}=F_{1}+\left(m_{\Lambda_{b}}+m_{\Lambda_{c}}\right)\left(\frac{F_{2}}{2 m_{\Lambda_{b}}}+\frac{F_{3}}{2 m_{\Lambda_{c}}}\right) \\
& f_{2}=-\frac{F_{2}}{2 m_{\Lambda_{b}}}-\frac{F_{3}}{2 m_{\Lambda_{c}}}, \quad f_{3}=\frac{F_{2}}{2 m_{\Lambda_{b}}}-\frac{F_{3}}{2 m_{\Lambda_{c}}} \\
& g_{1}=G_{1}-\left(m_{\Lambda_{b}}-m_{\Lambda_{c}}\right)\left(\frac{G_{2}}{2 m_{\Lambda_{b}}}+\frac{G_{3}}{2 m_{\Lambda_{c}}}\right) \\
& g_{2}=-\frac{G_{2}}{2 m_{\Lambda_{b}}}-\frac{G_{3}}{2 m_{\Lambda_{c}}}, \quad g_{3}=\frac{G_{2}}{2 m_{\Lambda_{b}}}-\frac{G_{3}}{2 m_{\Lambda_{c}}} .
\end{aligned}
$$

In terms of HQET form factors, up to order $\frac{1}{m_{Q}}$, one has

$F_{1}(w)=\xi_{\Lambda}(w)+\left(\frac{1}{2 m_{b}}+\frac{1}{2 m_{c}}\right)\left[B_{1}(w)-B_{2}(w)\right]$

$G_{1}(w)=\xi_{\Lambda}(w)+\left(\frac{1}{2 m_{b}}+\frac{1}{2 m_{c}}\right) B_{1}(w)$

$F_{2}(w)=G_{2}(w)=\frac{1}{2 m_{c}} B_{2}(w)$

$F_{3}(w)=-G_{3}(w)=\frac{1}{2 m_{b}} B_{2}(w)$,

where the $\frac{1}{m_{Q}}$ corrections read

$$
\begin{aligned}
& B_{1}(w)=\bar{\Lambda} \frac{w-1}{w+1} \xi_{\Lambda}(w)+A(w), \\
& B_{2}(w)=-\bar{\Lambda} \frac{2}{w+1} \xi_{\Lambda}(w) .
\end{aligned}
$$

The terms proportional to $\bar{\Lambda} \xi_{\Lambda}(w)$ correspond to the first order current perturbation in HQET, while the form factor $A(w)$ corresponds to the Lagrangian insertion perturbation,

$$
\begin{aligned}
& \left\langle\Lambda_{c}\left(p^{\prime}, s^{\prime}\right)\left|i \int d x T\left\{J(0), L_{1}(x)\right\}\right| \Lambda_{b}(p, s)\right\rangle \\
& \quad=A(w) \bar{u}_{\Lambda_{c}} \Gamma u_{\Lambda_{b}} .
\end{aligned}
$$

Luke's theorem [33] implies at zero recoil,

$$
B_{1}(1)=A(1)=0
$$

An interesting feature of formulas (A3), (A4) is that the form factors $f_{2}, f_{3}$ and $g_{2}, g_{3}$ are of the order $1 / m_{Q}^{2}$.

Let us finally give the notation for the form factors used in the lattice calculations [13]. In terms of the form factors (A1), (A2), the definition used in [13] is the following.

For the vector form factors,

$$
\begin{aligned}
& f_{+}=f_{1}+\frac{q^{2}}{m_{\Lambda_{b}}+m_{\Lambda_{c}}} f_{2} \\
& f_{\perp}=f_{1}+\left(m_{\Lambda_{b}}+m_{\Lambda_{c}}\right) f_{2} \\
& f_{0}=f_{1}+\frac{q^{2}}{m_{\Lambda_{b}}-m_{\Lambda_{c}}} f_{3},
\end{aligned}
$$

and for the axial form factors,

$$
\begin{aligned}
& g_{+}=g_{1}-\frac{q^{2}}{m_{\Lambda_{b}}-m_{\Lambda_{c}}} g_{2} \\
& g_{\perp}=g_{1}-\left(m_{\Lambda_{b}}-m_{\Lambda_{c}}\right) g_{2} \\
& g_{0}=g_{1}-\frac{q^{2}}{m_{\Lambda_{b}}+m_{\Lambda_{c}}} g_{3} .
\end{aligned}
$$

\section{Baryon form factors $\Lambda_{b} \rightarrow \Lambda_{c}\left(\frac{1}{2}^{-}\right)$up to the order $1 / m_{Q}$}

The matrix elements read

$$
\begin{aligned}
\left\langle\Lambda_{c}\left|\bar{c} \gamma_{\mu} b\right| \Lambda_{b}\right\rangle & =\bar{u}\left(p_{2}, s_{2}\right)\left[\gamma_{\mu} g_{1}^{V}\left(q^{2}\right)-i \sigma_{\mu \nu} q^{\nu} g_{2}^{V}\left(q^{2}\right)+q_{\mu} g_{3}^{V}\left(q^{2}\right)\right] \gamma_{5} u\left(p_{1}, s_{1}\right) \\
\left\langle\Lambda_{c}\left|\bar{c} \gamma_{\mu} \gamma_{5} b\right| \Lambda_{b}\right\rangle & =\bar{u}\left(p_{2}, s_{2}\right)\left[\gamma_{\mu} g_{1}^{A}\left(q^{2}\right)-i \sigma_{\mu \nu} q^{\nu} g_{2}^{A}\left(q^{2}\right)+q_{\mu} g_{3}^{A}\left(q^{2}\right)\right] u\left(p_{1}, s_{1}\right) .
\end{aligned}
$$

Notice the presence (absence) of $\gamma_{5}$ in the $V(A)$ matrix elements for $\Lambda_{b} \rightarrow \Lambda_{c}\left(\frac{1}{2}^{-}\right)$due to the intrinsic negative parity of the final state.

The alternative notation in terms of the four velocities is given by [35]

$$
\begin{aligned}
& \left\langle\Lambda_{c}^{1 / 2}\left(v^{\prime}, s^{\prime}\right)\left|\bar{c} \gamma^{\mu} b\right| \Lambda_{b}(v, s)\right\rangle=\bar{u}_{\Lambda_{c}}\left(v^{\prime}, s^{\prime}\right)\left[d_{V_{1}} \gamma^{\mu}+d_{V_{2}} v^{\mu}+d_{V_{3}} v^{\prime \mu}\right] \gamma_{5} u_{\Lambda_{b}}(v, s) \\
& \left\langle\Lambda_{c}\left(v^{\prime}, s^{\prime}\right)\left|\bar{c} \gamma^{\mu} b \gamma_{5}\right| \Lambda_{b}(v, s)\right\rangle=\bar{u}_{\Lambda_{c}}\left(v^{\prime}, s^{\prime}\right)\left[d_{A_{1}} \gamma^{\mu}+d_{A_{2}} v^{\mu}+d_{A_{3}} v^{\prime \mu}\right] u_{\Lambda_{b}}(v, s),
\end{aligned}
$$

and the relation between both notations is 


$$
\begin{aligned}
& g_{1}^{V}=d_{1}^{V}-\left(m_{\Lambda_{b}}-m_{\Lambda_{c}}\right)\left(\frac{d_{2}^{V}}{2 m_{\Lambda_{b}}}+\frac{d_{3}^{V}}{2 m_{\Lambda_{c}}}\right) \\
& g_{2}^{V}=-\frac{d_{2}^{V}}{2 m_{\Lambda_{b}}}-\frac{d_{3}^{V}}{2 m_{\Lambda_{c}}}, \quad g_{3}^{V}=\frac{d_{2}^{V}}{2 m_{\Lambda_{b}}}-\frac{d_{3}^{V}}{2 m_{\Lambda_{c}}} \\
& g_{1}^{A}=d_{1}^{A}+\left(m_{\Lambda_{b}}+m_{\Lambda_{c}}\right)\left(\frac{d_{2}^{A}}{2 m_{\Lambda_{b}}}+\frac{d_{3}^{A}}{2 m_{\Lambda_{c}}}\right) \\
& g_{2}^{A}=-\frac{d_{2}^{A}}{2 m_{\Lambda_{b}}}-\frac{d_{3}^{A}}{2 m_{\Lambda_{c}}}, \quad g_{3}^{A}=\frac{d_{2}^{A}}{2 m_{\Lambda_{b}}}-\frac{d_{3}^{A}}{2 m_{\Lambda_{c}}} .
\end{aligned}
$$

Neglecting for the moment the subleading terms dependent on Lagrangian insertions and keeping only the subleading $1 / m_{Q}$ terms that are proportional to the inelastic IW function $\sigma(w)$, the form factors (A11) are given by the expressions of Leibovich and Stewart [35],

$$
\begin{aligned}
& d_{1}^{V}=\frac{1}{\sqrt{3}}\left[(w-1) \sigma+\epsilon_{c} 3\left(w \bar{\Lambda}^{\prime}-\bar{\Lambda}\right) \sigma-\epsilon_{b}\left(\bar{\Lambda}^{\prime}-w \bar{\Lambda}\right) \sigma\right] \\
& d_{2}^{V}=\frac{1}{\sqrt{3}}\left[-2 \sigma-\epsilon_{b}\left(\bar{\Lambda}^{\prime}+\bar{\Lambda}\right) \sigma\right], \quad d_{3}^{V}=\frac{1}{\sqrt{3}}\left[\epsilon_{b}\left(\bar{\Lambda}^{\prime}+\bar{\Lambda}\right) \sigma\right] \\
& d_{1}^{A}=\frac{1}{\sqrt{3}}\left[(w+1) \sigma+\epsilon_{c} 3\left(w \bar{\Lambda}^{\prime}-\bar{\Lambda}\right) \sigma-\epsilon_{b}\left(\bar{\Lambda}^{\prime}-w \bar{\Lambda}\right) \sigma\right] \\
& d_{2}^{A}=\frac{1}{\sqrt{3}}\left[-2 \sigma+2 \epsilon_{b}\left(\bar{\Lambda}^{\prime}-\bar{\Lambda}\right) \sigma\right], \\
& d_{3}^{A}=\frac{1}{\sqrt{3}}\left[2 \epsilon_{b}\left(\bar{\Lambda}^{\prime}-\bar{\Lambda}\right) \sigma\right] .
\end{aligned}
$$

The subleading Lagrangian perturbations give the following extra contributions to the preceding form factors [35]:

$$
\begin{aligned}
\Delta d_{1}^{V}= & \frac{1}{\sqrt{3}}\left\{\epsilon_{c}\left[-2\left(w^{2}-1\right) \sigma_{1}+(w-1)\left(\phi_{\text {kin }}^{(c)}-2 \phi_{\text {mag }}^{(c)}\right)\right]\right. \\
& \left.-\epsilon_{b}\left[-(w-1) \phi_{\text {kin }}^{(b)}\right]\right\} \\
\Delta d_{2}^{V}= & \frac{1}{\sqrt{3}}\left\{2 \epsilon_{c}\left[-\left(\phi_{\text {kin }}^{(c)}-2 \phi_{\text {mag }}^{(c)}\right)\right]\right. \\
& \left.-2 \epsilon_{b}\left[-(w+1) \sigma_{1}+\phi_{\text {kin }}^{(b)}+\phi_{\text {mag }}^{(b)}\right]\right\} \\
\Delta d_{3}^{V}= & \frac{1}{\sqrt{3}} 2 \epsilon_{b}\left[-(w+1) \sigma_{1}-\phi_{\text {mag }}^{(b)}\right] \\
\Delta d_{1}^{A}= & \frac{1}{\sqrt{3}}\left\{\epsilon_{c}\left[-2\left(w^{2}-1\right) \sigma_{1}+(w+1)\left(\phi_{\text {kin }}^{(c)}-2 \phi_{\text {mag }}^{(c)}\right)\right]\right. \\
& \left.-\epsilon_{b}\left[-(w+1) \phi_{\text {kin }}^{(b)}\right]\right\} \\
\Delta d_{2}^{A}= & \frac{1}{\sqrt{3}}\left\{-2 \epsilon_{c}\left[\phi_{\text {kin }}^{(c)}-2 \phi_{\text {mag }}^{(c)}\right]+2 \epsilon_{b}\left[-\phi_{\text {kin }}^{(b)}+\phi_{\text {mag }}^{(b)}\right]\right\} \\
\Delta d_{3}^{A}= & \frac{1}{\sqrt{3}} 2 \epsilon_{b}\left[-(w-1) \sigma_{1}-\phi_{\text {mag }}^{(b)}\right] .
\end{aligned}
$$

According to Leibovich and Stewart, the chromomagnetic functions $\phi_{m a g}^{(Q)}$ are expected to be small because the
$j^{P}=1^{-}$doublet mass splittings are small, and they are taken

$$
\phi_{m a g}^{(Q)}=0 \quad(Q=c, b) .
$$

The functions $\phi_{k i n}^{(Q)}$ can be absorbed by the Isgur-Wise function by replacing $\sigma$ with

$$
\tilde{\sigma}(w)=\sigma(w)+\epsilon_{c} \phi_{k i n}^{(c)}(w)+\epsilon_{b} \phi_{k i n}^{(b)}(w) .
$$

Moreover, [35] assumes

$$
\phi_{\text {kin }}^{(c)}(1)=0,
$$

as predicted by QCD in the large $N_{c}$ limit, and therefore,

$$
\tilde{\sigma}(1) \simeq \sigma(1) .
$$

One is left then with two IW functions, $\tilde{\sigma}(w)$ and $\sigma_{1}(w)$, and defining the ratio,

$$
\hat{\sigma}_{1}(w)=\frac{\sigma_{1}(w)}{\tilde{\sigma}(w)} .
$$

Leibovich and Stewart assume a constant ratio for $\hat{\sigma}_{1}(w)=$ constant $=\hat{\sigma}_{1}$ within the range,

$$
-1 \mathrm{GeV}<\hat{\sigma}_{1}<1 \mathrm{GeV}
$$

\section{APPENDIX B: THE $Q q q$ ELASTIC IW FUNCTION $\xi_{\Lambda}(w)$ IN THE BT SCHEME}

Let us begin with the general formula for a transition matrix element in the Bakamjian-Thomas relativistic quark model in terms of $2 \times 2$ matrices [15],

$$
\begin{aligned}
\left\langle\mathbf{P}^{\prime}|O| \mathbf{P}\right\rangle= & \int \prod_{i=2}^{n} \frac{d \mathbf{p}_{i}}{(2 \pi)^{3}} \sqrt{\frac{\sum_{j} p_{j}^{\prime 0} \sum_{k} p_{k}^{0}}{M_{0}^{\prime} M_{0}}} \\
& \times \prod_{i=1}^{n} \sqrt{\frac{k_{i}^{\prime 0} k_{i}^{0}}{p_{i}^{\prime 0} p_{i}^{0}}} \sum_{s_{1}^{\prime} \ldots s_{n}^{\prime}} \sum_{s_{1} \ldots s_{n}} \varphi_{s_{1}^{\prime} \ldots s_{n}^{\prime}}^{\prime}\left(\mathbf{k}_{2}^{\prime} \ldots \mathbf{k}_{n}^{\prime}\right)^{*} \\
& \times\left[D_{1}^{\prime}\left(\mathbf{R}_{1}^{-1}\right) O\left(\mathbf{p}_{1}^{\prime}, \mathbf{p}_{1}\right) D_{1}\left(\mathbf{R}_{1}\right]_{s_{1}^{\prime}, s_{1}}\right. \\
& \times \prod_{i=2}^{n} D_{i}\left(\mathbf{R}_{i}^{\prime-1} \mathbf{R}_{i}\right)_{s_{i}^{\prime} \ldots s_{i}} \varphi_{s_{1} \ldots s_{n}}\left(\mathbf{k}_{2} \ldots \mathbf{k}_{n}\right),
\end{aligned}
$$

where 1 labels the active quark, the matrix element of the currrent operator $O$ is

$$
O\left(\mathbf{p}^{\prime}, \mathbf{p}\right)_{s^{\prime}, s}=\left\langle\mathbf{p}^{\prime}, s^{\prime}|O| \mathbf{p}, s\right\rangle,
$$

and the vectors $\mathbf{k}_{i}$, the 0 components $k_{i}^{0}$ and $p_{i}^{0}, M_{0}$ and the Wigner rotations $\mathbf{R}_{i}$ are functions of the $\mathbf{p}_{i}$ defined as follows: 


$$
\begin{aligned}
p_{i}^{0} & =\sqrt{\mathbf{p}_{i}^{2}+m_{i}^{2}}, \quad M_{0}=\sqrt{\left(\Sigma p_{j}\right)^{2}}, \\
k_{i} & =\mathbf{B}_{\Sigma p_{j}}^{-1} p_{i}, \quad \mathbf{R}_{i}=\mathbf{B}_{p_{i}}^{-1} \mathbf{B}_{\Sigma p_{j}} \mathbf{B}_{k_{i}},
\end{aligned}
$$

where $\mathbf{B}_{p}$ is the boost $\left(\sqrt{p^{2}}, \mathbf{0}\right) \rightarrow p$ and $D_{i}(\mathbf{R})$ is the matrix of rotation $\mathbf{R}$ for the spin $S_{i}$.

The internal wave function of the baryon $\Lambda_{Q}$ with heavy quarks $Q=b$ or $c$ and polarization $\mu$ will write

$$
\varphi_{s_{1}, s_{2}, s_{3}}^{(\mu)}\left(\mathbf{k}_{2}, \mathbf{k}_{3}\right)=\chi_{s_{1}}^{(\mu)} \frac{i}{\sqrt{2}}\left(\sigma_{2}\right)_{s_{2}, s_{3}} \varphi\left(\mathbf{k}_{2}, \mathbf{k}_{3}\right)
$$

because the spectator quarks 2,3 are in a state of spin and isospin 0 , and the notation $\chi_{s_{1}}^{(\mu)}$ for the active quark means $\chi_{+1 / 2}^{(+1 / 2)}=\chi_{-1 / 2}^{(-1 / 2)}=1$ and $\chi_{-1 / 2}^{(+1 / 2)}=\chi_{+1 / 2}^{(-1 / 2)}=0$.

Considering the polarized states $\Lambda_{Q}$, the matrix element (B1) writes then

$$
\begin{aligned}
\left\langle\mathbf{P}^{\prime}, \mu^{\prime}|O| \mathbf{P}, \mu\right\rangle= & \int \frac{d \mathbf{p}_{2}}{(2 \pi)^{3}} \frac{d \mathbf{p}_{3}}{(2 \pi)^{3}} \sqrt{\frac{\sum_{j} p_{j}^{\prime 0} \sum_{k} p_{k}^{0}}{M_{0}^{\prime} M_{0}}} \\
& \times \prod_{i=1}^{3} \sqrt{\frac{k_{i}^{\prime 0} k_{i}^{0}}{p_{i}^{\prime 0} p_{i}^{0}}} \sum_{s_{1}^{\prime}, s_{2}^{\prime}, s_{3}^{\prime}} \sum_{s_{1}, s_{2}, s_{3}} \varphi_{s_{1}^{\prime}, s_{2}^{\prime}, s_{3}^{\prime}}^{\left(\mu^{\prime}\right.}\left(\mathbf{k}_{2}^{\prime}, \mathbf{k}_{3}^{\prime}\right)^{*} \\
& \times\left[D_{1}^{\prime}\left(\mathbf{R}_{1}^{\prime-1}\right) O\left(\mathbf{p}_{1}^{\prime}, \mathbf{p}_{1}\right) D_{1}\left(\mathbf{R}_{1}\right)\right]_{s_{1}^{\prime}, s_{1}} \\
& \times \prod_{i=2}^{3} D_{i}\left(\mathbf{R}_{i}^{\prime-1} \mathbf{R}_{i}\right)_{s_{i}^{\prime} \ldots s_{i}} \varphi_{s_{1}, s_{2}, s_{3}}^{(\mu)}\left(\mathbf{k}_{2}, \mathbf{k}_{3}\right)
\end{aligned}
$$

From the wave function (B4), one gets

$$
\begin{aligned}
\left\langle\mathbf{P}^{\prime}, \mu^{\prime}|O| \mathbf{P}, \mu\right\rangle= & \frac{1}{2} \int \frac{d \mathbf{p}_{2}}{(2 \pi)^{3}} \frac{d \mathbf{p}_{3}}{(2 \pi)^{3}} \sqrt{\frac{\sum_{j} p_{j}^{\prime 0} \sum_{k} p_{k}^{0}}{M_{0}^{\prime} M_{0}}} \\
& \times \prod_{i=1}^{3} \sqrt{\frac{k_{i}^{\prime 0} k_{i}^{0}}{p_{i}^{\prime 0} p_{i}^{0}}} \varphi\left(\mathbf{k}_{2}^{\prime}, \mathbf{k}_{3}^{\prime}\right)^{*} \varphi\left(\mathbf{k}_{2}, \mathbf{k}_{3}\right) \\
& \times\left(\chi^{\left(\mu^{\prime}\right) \dagger} D_{1}^{\prime}\left(\mathbf{R}_{1}^{\prime-1}\right) O\left(\mathbf{p}_{1}^{\prime}, \mathbf{p}_{1}\right) D_{1}\left(\mathbf{R}_{1}\right) \chi^{(\mu)}\right) \\
& \times \operatorname{Tr}\left[D_{2}\left(\mathbf{R}_{2}^{\prime-1} \mathbf{R}_{2}\right)^{t} \sigma_{2} D_{3}\left(\mathbf{R}_{3}^{\prime-1} \mathbf{R}_{3}\right) \sigma_{2}\right],
\end{aligned}
$$

and using the relation,

$$
\sigma_{2} D(\mathbf{R}) \sigma_{2}=D\left(\mathbf{R}^{-1}\right)^{t},
$$

one obtains

$$
\begin{aligned}
\left\langle\mathbf{P}^{\prime}, \mu^{\prime}|O| \mathbf{P}, \mu\right\rangle= & \frac{1}{2} \int \frac{d \mathbf{p}_{2}}{(2 \pi)^{3}} \frac{d \mathbf{p}_{3}}{(2 \pi)^{3}} \sqrt{\frac{\sum_{j} p_{j}^{\prime 0} \sum_{k} p_{k}^{0}}{M_{0}^{\prime} M_{0}}} \\
& \times \prod_{i=1}^{3} \sqrt{\frac{k_{i}^{\prime 0} k_{i}^{0}}{p_{i}^{\prime 0} p_{i}^{0}}} \varphi\left(\mathbf{k}_{2}^{\prime}, \mathbf{k}_{3}^{\prime}\right)^{*} \varphi\left(\mathbf{k}_{2}, \mathbf{k}_{3}\right) \\
& \times\left(\chi^{\left(\mu^{\prime}\right) \dagger} D\left(\mathbf{R}_{1}^{\prime-1}\right) O\left(\mathbf{p}_{1}^{\prime}, \mathbf{p}_{1}\right) D\left(\mathbf{R}_{1}\right) \chi^{(\mu)}\right) \\
& \times \operatorname{Tr}\left[D\left(\mathbf{R}_{3}^{-1} \mathbf{R}_{3}^{\prime} \mathbf{R}_{2}^{\prime-1} \mathbf{R}_{2}\right)\right],
\end{aligned}
$$

where we have omitted the index indicating on which quark the Wigner rotation acts and keep it only on the rotation, because all these matrices act on the spin $\frac{1}{2}$. Equation (B6) is the final formula in the $2 \times 2$ matrix formalism and at a finite mass.

We now pass to a $4 \times 4$ matrix formulation,

$$
\begin{aligned}
O\left(\mathbf{p}_{1}^{\prime}, \mathbf{p}_{1}\right) & \rightarrow \sqrt{\frac{m_{1}^{\prime} m_{1}}{p_{1}^{\prime 0} p_{1}^{0}}} \frac{1+\gamma^{0}}{2} \mathbf{B}_{p_{1}^{\prime}}^{-1} O \mathbf{B}_{p_{1}}^{-1} \frac{1+\gamma^{0}}{2}, \\
\chi^{(\mu)} & \rightarrow \frac{1+\gamma^{0}}{2} \chi^{(\mu)},
\end{aligned}
$$

and will have, for the spinor matrix element,

$$
\begin{aligned}
& \left(\chi^{\left(\mu^{\prime}\right) \dagger} D_{1}^{\prime}\left(\mathbf{R}_{1}^{\prime-1}\right) O\left(\mathbf{p}_{1}^{\prime}, \mathbf{p}_{1}\right) D_{1}\left(\mathbf{R}_{1}\right) \chi^{(\mu)}\right) \\
& \quad=\frac{1}{4} \sqrt{\frac{m_{1}^{\prime} m_{1}}{p_{1}^{\prime 0} p_{1}^{0}}}\left(\chi^{\left(\mu^{\prime}\right) \dagger}\left(1+\gamma^{0}\right) \mathbf{B}_{k_{1}^{\prime}}^{-1} \mathbf{B}_{u^{\prime}}^{-1} O \mathbf{B}_{u} \mathbf{B}_{k_{1}}\left(1+\gamma^{0}\right) \chi^{(\mu)}\right),
\end{aligned}
$$

since $\frac{1+\gamma^{0}}{2}$ commutes with the Wigner rotations, and we have made explicit the rotations in terms of boost matrices according to (B3). In the last equation, $O$ denotes simply the Dirac matrix in the current $\bar{c} O b$.

In terms of the boosted spinors,

$$
\chi_{u}^{(\mu)}=\mathbf{B}_{u} \chi^{(\mu)}, \quad \bar{\chi}_{u}^{\left(\mu^{\prime}\right)}=\bar{\chi}^{\left(\mu^{\prime}\right)} \mathbf{B}_{u^{\prime}}^{-1},
$$

the spinor matrix element in (B10) writes

$$
\begin{aligned}
& \left(\chi^{\left(\mu^{\prime}\right) \dagger}\left(1+\gamma^{0}\right) \mathbf{B}_{k_{1}^{\prime}}^{-1} \mathbf{B}_{u^{\prime}}^{-1} O \mathbf{B}_{u} \mathbf{B}_{k_{1}}\left(1+\gamma^{0}\right) \chi^{(\mu)}\right) \\
& \quad=\left(\bar{\chi}_{u^{\prime}}^{\left(\mu^{\prime}\right)} \mathbf{B}_{u^{\prime}}\left(1+\gamma^{0}\right) \mathbf{B}_{k_{1}^{\prime}}^{-1} \mathbf{B}_{u^{\prime}}^{-1} O \mathbf{B}_{u} \mathbf{B}_{k_{1}}\left(1+\gamma^{0}\right) \mathbf{B}_{u}^{-1} \chi_{u}^{(\mu)}\right)
\end{aligned}
$$

and using the identities,

$$
\begin{aligned}
\mathbf{B}_{u} \mathbf{B}_{k_{1}}\left(1+\gamma^{0}\right) \mathbf{B}_{u}^{-1} & =\frac{\left(m_{1}+\not p_{1}\right)(1+\not \iota)}{\sqrt{2 m_{1}\left(k_{1}^{0}+m_{1}\right)}}, \\
\mathbf{B}_{u^{\prime}}\left(1+\gamma^{0}\right) \mathbf{B}_{k_{1}^{\prime}}^{-1} \mathbf{B}_{u^{\prime}}^{-1} & =\frac{\left(1+\not \iota^{\prime}\right)\left(m_{1}^{\prime}+\not p_{1}^{\prime}\right)}{\sqrt{2 m_{1}^{\prime}\left(k_{1}^{\prime 0}+m_{1}^{\prime}\right)}},
\end{aligned}
$$


one gets the formula in the $4 \times 4$ form,

$$
\begin{aligned}
\left(\chi^{\left(\mu^{\prime}\right) \dagger} D_{1}^{\prime}\left(\mathbf{R}_{1}^{\prime-1}\right) O\left(\mathbf{p}_{1}^{\prime}, \mathbf{p}_{1}\right) D_{1}\left(\mathbf{R}_{1}\right) \chi^{(\mu)}\right)= & \frac{1}{4} \sqrt{\frac{m_{1}^{\prime} m_{1}}{p_{1}^{\prime 0} p_{1}^{0}}} \frac{1}{\sqrt{2 m_{1}\left(k_{1}^{0}+m_{1}\right)}} \frac{1}{\sqrt{2 m_{1}^{\prime}\left(k_{1}^{\prime 0}+m_{1}^{\prime}\right)}} \\
& \times\left(\bar{\chi}_{u^{\prime}}^{\left(\mu^{\prime}\right)}\left(1+\not \mu^{\prime}\right)\left(m_{1}^{\prime}+\not p_{1}^{\prime}\right) O\left(m_{1}+\not p_{1}\right)(1+\not h) \chi_{u}^{(\mu)}\right) .
\end{aligned}
$$

We have now to compute the trace in formula (B8) that reads, in the $4 \times 4$ Dirac matrix formalism and in terms of the boost matrices,

$$
\begin{aligned}
\operatorname{Tr}\left[D\left(\mathbf{R}_{3}^{-1} \mathbf{R}_{3}^{\prime} \mathbf{R}_{2}^{\prime-1} \mathbf{R}_{2}\right)\right] & \rightarrow \frac{1}{2} \operatorname{Tr}\left[\left(1+\gamma^{0}\right) \mathbf{R}_{3}^{-1} \mathbf{R}_{3}^{\prime} \mathbf{R}_{2}^{\prime-1} \mathbf{R}_{2}\right] \\
& =\frac{1}{8} \operatorname{Tr}\left[\left(1+\gamma^{0}\right) \mathbf{B}_{k_{3}}^{-1} \mathbf{B}_{u}^{-1} \mathbf{B}_{p_{3}} \mathbf{B}_{p_{3}^{\prime}}^{-1} \mathbf{B}_{u^{\prime}} \mathbf{B}_{k_{3}^{\prime}}\left(1+\gamma^{0}\right) \mathbf{B}_{k_{2}^{\prime}}^{-1} \mathbf{B}_{u^{\prime}}^{-1} \mathbf{B}_{p_{2}^{\prime}} \mathbf{B}_{p_{2}}^{-1} \mathbf{B}_{u} \mathbf{B}_{k_{2}}\left(1+\gamma^{0}\right)\right] \\
& =\frac{1}{16} \operatorname{Tr}\left[\mathbf{B}_{u}\left(1+\gamma^{0}\right) \mathbf{B}_{k_{3}}^{-1} \mathbf{B}_{u}^{-1} \mathbf{B}_{u^{\prime}} \mathbf{B}_{k_{3}^{\prime}}\left(1+\gamma^{0}\right) \mathbf{B}_{u^{\prime}}^{-1} \mathbf{B}_{u^{\prime}}\left(1+\gamma^{0}\right) \mathbf{B}_{k_{2}^{\prime}}^{-1} \mathbf{B}_{u^{\prime}}^{-1} \mathbf{B}_{u} \mathbf{B}_{k_{2}}\left(1+\gamma^{0}\right) \mathbf{B}_{u}^{-1}\right],
\end{aligned}
$$

because $1+\gamma^{0}$ commutes with the Wigner rotations, the quarks 2,3 are spectators, and then one has $p_{2}=p_{2}^{\prime}$ and $p_{3}=p_{3}^{\prime}$, and we have inserted the products $\mathbf{B}_{u}^{-1} \mathbf{B}_{u}=\mathbf{B}_{u^{\prime}}^{-1} \mathbf{B}_{u^{\prime}}=1$ within the trace.

We now use relations of the type (B13) and $(1+\not h)(1+\not h)=2(1+\not h),\left(m+\not p_{2}\right)\left(m+\not p_{2}\right)=2 m\left(m+\not p_{2}\right) \ldots$, and one finally gets

$$
\operatorname{Tr}\left[D\left(\mathbf{R}_{3}^{-1} \mathbf{R}_{3}^{\prime} \mathbf{R}_{2}^{\prime-1} \mathbf{R}_{2}\right)\right]=\frac{1}{4} \frac{1}{\sqrt{\left(k_{2}^{0}+m\right)\left(k_{2}^{\prime 0}+m\right)\left(k_{3}^{0}+m\right)\left(k_{3}^{\prime 0}+m\right)}} \operatorname{Tr}\left[(1+\not \iota)\left(m+\not p_{2}\right)\left(1+\not \iota^{\prime}\right)\left(m+\not p_{3}\right)\right] .
$$

The computation of the trace finally gives

$$
\begin{aligned}
\operatorname{Tr}\left[D\left(\mathbf{R}_{3}^{-1} \mathbf{R}_{3}^{\prime} \mathbf{R}_{2}^{\prime-1} \mathbf{R}_{2}\right)\right]= & \frac{1}{\sqrt{\left(k_{2}^{0}+m\right)\left(k_{2}^{\prime 0}+m\right)\left(k_{3}^{0}+m\right)\left(k_{3}^{\prime 0}+m\right)}} \\
& \times\left[m^{2}\left(1+u \cdot u^{\prime}\right)+m\left(u+u^{\prime}\right) \cdot\left(p_{2}+p_{3}\right)+p_{2} \cdot p_{3}+\left(u \cdot p_{2}\right)\left(u^{\prime} \cdot p_{3}\right)+\left(u \cdot p_{3}\right)\left(u^{\prime} \cdot p_{2}\right)-\left(u \cdot u^{\prime}\right)\left(p_{2} \cdot p_{3}\right)\right] .
\end{aligned}
$$

So one gets finally the matrix element,

$$
\begin{aligned}
\left\langle\mathbf{P}^{\prime}, \mu^{\prime}|O| \mathbf{P}, \mu\right\rangle= & \frac{1}{8} \int \frac{d \mathbf{p}_{2}}{(2 \pi)^{3}} \frac{d \mathbf{p}_{3}}{(2 \pi)^{3}} \sqrt{\frac{\sum_{j} p_{j}^{\prime 0} \sum_{k} p_{k}^{0}}{M_{0}^{\prime} M_{0}}} \prod_{i=1}^{3} \sqrt{\frac{k_{i}^{\prime 0} k_{i}^{0}}{p_{i}^{\prime 0} p_{i}^{0}}} \varphi\left(\mathbf{k}_{2}^{\prime}, \mathbf{k}_{3}^{\prime}\right)^{*} \varphi\left(\mathbf{k}_{2}, \mathbf{k}_{3}\right) \\
& \times \frac{1}{\sqrt{p_{1}^{\prime 0} p_{1}^{0}}} \frac{1}{\sqrt{\left(k_{1}^{0}+m_{1}\right)\left(k_{1}^{\prime 0}+m_{1}^{\prime}\right)}} \frac{1}{\sqrt{\left(k_{2}^{0}+m\right)\left(k_{2}^{\prime 0}+m\right)\left(k_{3}^{0}+m\right)\left(k_{3}^{\prime 0}+m\right)}} \\
& \times\left[m^{2}\left(1+u \cdot u^{\prime}\right)+m\left(u+u^{\prime}\right) \cdot\left(p_{2}+p_{3}\right)+p_{2} \cdot p_{3}+\left(u \cdot p_{2}\right)\left(u^{\prime} \cdot p_{3}\right)+\left(u \cdot p_{3}\right)\left(u^{\prime} \cdot p_{2}\right)-\left(u \cdot u^{\prime}\right)\left(p_{2} \cdot p_{3}\right)\right] \\
& \times\left(\bar{\chi}_{u^{\prime}}^{\left(\mu^{\prime}\right)}\left(m_{1}^{\prime}+p_{1}^{\prime}\right) O\left(m_{1}+\not p_{1}\right) \chi_{u}^{(\mu)}\right),
\end{aligned}
$$

because $(1+\not h) \chi_{u}^{(\mu)}=2 \chi_{u}^{(\mu)}, \quad \bar{\chi}_{u^{\prime}}^{\left(\mu^{\prime}\right)}\left(1+\not \mu^{\prime}\right)=2 \bar{\chi}_{u^{\prime}}^{\left(\mu^{\prime}\right)}$.

In the heavy quark limit [15], one has

$$
\begin{aligned}
\left(u, u^{\prime}\right) & \rightarrow\left(v, v^{\prime}\right), \quad\left(\frac{p_{1}}{m_{1}}, \frac{p_{1}^{\prime}}{m_{1}^{\prime}}\right) \rightarrow\left(v, v^{\prime}\right), & \frac{\sum_{j} p_{j}^{\prime 0} \sum_{k} p_{k}^{0}}{M_{0}^{\prime} M_{0}} \rightarrow v^{0} v^{\prime 0} \\
\frac{k_{1}^{0}}{m_{1}}, \frac{k_{1}^{\prime 0}}{m_{1}^{\prime}} \rightarrow 1, & \left(k_{2}^{0}, k_{2}^{\prime 0}\right) \rightarrow\left(p_{2} \cdot v, p_{2} \cdot v^{\prime}\right), & \left(k_{3}^{0}, k_{3}^{\prime 0}\right) \rightarrow\left(p_{3} \cdot v, p_{3} \cdot v^{\prime}\right),
\end{aligned}
$$


and since $p_{2}=p_{2}^{\prime}, p_{3}=p_{3}^{\prime}$ for the spectator quarks, one gets the heavy quark limit matrix element,

$$
\begin{aligned}
\left\langle\mathbf{P}^{\prime}, \mu^{\prime}|O| \mathbf{P}, \mu\right\rangle= & \frac{1}{\sqrt{v^{0} v^{\prime 0}}} \int \frac{d \mathbf{p}_{2}}{(2 \pi)^{3}} \frac{1}{p_{2}^{0}} \frac{d \mathbf{p}_{3}}{(2 \pi)^{3}} \frac{1}{p_{3}^{0}} \varphi\left(\mathbf{k}_{2}^{\prime}, \mathbf{k}_{3}^{\prime}\right)^{*} \varphi\left(\mathbf{k}_{2}, \mathbf{k}_{3}\right) \\
& \times \frac{1}{2} \frac{\sqrt{\left(p_{2} \cdot v\right)\left(p_{3} \cdot v\right)\left(p_{2} \cdot v^{\prime}\right)\left(p_{3} \cdot v^{\prime}\right)}}{\sqrt{\left(p_{2} \cdot v+m\right)\left(p_{3} \cdot v+m\right)\left(p_{2} \cdot v^{\prime}+m\right)\left(p_{3} \cdot v^{\prime}+m\right)}} \\
& \times\left[m^{2}\left(1+v \cdot v^{\prime}\right)+m\left(v+v^{\prime}\right) \cdot\left(p_{2}+p_{3}\right)+\left(p_{2} \cdot v\right)\left(p_{3} \cdot v^{\prime}\right)+\left(p_{3} \cdot v\right)\left(p_{2} \cdot v^{\prime}\right)+\left(p_{2} \cdot p_{3}\right)\left(1-v \cdot v^{\prime}\right)\right] \\
& \times\left(\bar{\chi}_{v^{\prime}}^{\left(\mu^{\prime}\right)} O \chi_{v}^{(\mu)}\right) .
\end{aligned}
$$

Finally, identifying with the definition of the Isgur-Wise function within the same normalization convention,

$$
\left\langle\Lambda_{b}\left(\mathbf{P}^{\prime}, \mu^{\prime}\right)|O| \Lambda_{c}(\mathbf{P}, \mu)\right\rangle=\frac{1}{\sqrt{v^{0} v^{0}}} \xi_{\Lambda}\left(v \cdot v^{\prime}\right)\left(\bar{\chi}_{v^{\prime}}^{\left(\mu^{\prime}\right)} O \chi_{v}^{(\mu)}\right)
$$

one gets

$$
\begin{aligned}
\xi_{\Lambda}\left(v \cdot v^{\prime}\right)= & \int \frac{d \mathbf{p}_{2}}{(2 \pi)^{3}} \frac{1}{p_{2}^{0}} \frac{d \mathbf{p}_{3}}{(2 \pi)^{3}} \frac{1}{p_{3}^{0}} \sqrt{\left(p_{2} \cdot v\right)\left(p_{3} \cdot v\right)\left(p_{2} \cdot v^{\prime}\right)\left(p_{3} \cdot v^{\prime}\right)} \varphi\left(\mathbf{k}_{2}^{\prime}, \mathbf{k}_{3}^{\prime}\right)^{*} \varphi\left(\mathbf{k}_{2}, \mathbf{k}_{3}\right) \\
& \times \frac{\left[m^{2}\left(1+v \cdot v^{\prime}\right)+m\left(v+v^{\prime}\right) \cdot\left(p_{2}+p_{3}\right)+\left(p_{2} \cdot v\right)\left(p_{3} \cdot v^{\prime}\right)+\left(p_{3} \cdot v\right)\left(p_{2} \cdot v^{\prime}\right)+\left(p_{2} \cdot p_{3}\right)\left(1-v \cdot v^{\prime}\right)\right]}{2 \sqrt{\left(p_{2} \cdot v+m\right)\left(p_{3} \cdot v+m\right)\left(p_{2} \cdot v^{\prime}+m\right)\left(p_{3} \cdot v^{\prime}+m\right)}},
\end{aligned}
$$

where the arguments of the internal wave function are the three-dimensional parts of the four vectors,

$$
k_{i}=\mathbf{B}_{v}^{-1} p_{i}, \quad k_{i}^{\prime}=\mathbf{B}_{v^{\prime}}^{-1} p_{i} \quad(i=2,3) .
$$

The factor $\sqrt{\left(p_{2} \cdot v\right)\left(p_{3} \cdot v\right)\left(p_{2} \cdot v^{\prime}\right)\left(p_{3} \cdot v^{\prime}\right)}$ in the first line of (B21) comes from the Jacobian, and the last line comes from the Wigner rotations.

One can observe that the expression of the Isgur-Wise function (B21) is fully covariant, in particular due to the Lorentz invariant measures $\frac{d \mathbf{p}_{i}}{p_{i}^{0}}(i=2,3)$.

As we will see below, to get covariance of the IW function, one needs, in (B21), rotational invariance of the internal wave functions.

For $v \cdot v^{\prime}=1$, one finds that, due to the normalization of the internal wave function, the Isgur-Wise function is correctly normalized,

$$
\xi_{\Lambda}(1)=\int \frac{d \mathbf{p}_{2}}{(2 \pi)^{3}} \frac{d \mathbf{p}_{3}}{(2 \pi)^{3}}\left|\varphi\left(\mathbf{p}_{2}, \mathbf{p}_{3}\right)\right|^{2}=1 .
$$

The expressions of the IW functions in the baryon case (B21) contain Lorentz invariant measures $\frac{d \mathbf{p}_{i}}{p_{i}^{0}}$ and a Lorentz invariant kernel. However, we have the product of wave functions $\varphi\left(\mathbf{k}_{2}^{\prime}, \mathbf{k}_{3}^{\prime}\right)^{*} \varphi\left(\mathbf{k}_{2}, \mathbf{k}_{3}\right)$ and to show that both IW functions are Lorentz invariant, we need to demonstrate that these products are also Lorentz invariant.

Consider now the product of wave functions,

$$
\begin{aligned}
& \varphi\left(\mathbf{k}_{2}^{\prime}, \mathbf{k}_{3}^{\prime}\right)^{*} \varphi\left(\mathbf{k}_{2}, \mathbf{k}_{3}\right) \\
& \quad=\varphi\left(\overrightarrow{\mathbf{B}_{v^{\prime}}^{-1} p_{2}}, \overleftrightarrow{\mathbf{B}_{v^{\prime}}^{-1} p_{3}}\right)^{*} \varphi\left(\overrightarrow{\mathbf{B}_{v}^{-1} p_{2}}, \overleftrightarrow{\mathbf{B}_{v}^{-1} p_{2}}\right) .
\end{aligned}
$$

The radial wave functions are rotational invariant, so that they can be redefined as follows:

$$
\varphi\left(\mathbf{k}_{2}, \mathbf{k}_{3}\right)=\psi\left(\mathbf{k}_{2}^{2}, \mathbf{k}_{3}^{2}, \mathbf{k}_{2} \cdot \mathbf{k}_{3}\right)
$$

and similarly, for $\varphi\left(\mathbf{k}_{2}^{\prime}, \mathbf{k}_{3}^{\prime}\right)$.

One has

$\mathbf{k}_{2}^{2}=\left(\overrightarrow{\mathbf{B}_{v}^{-1} p_{2}}\right)^{2}=\left(\left(\mathbf{B}_{v}^{-1} p_{2}\right)^{0}\right)^{2}-m^{2}=\left(p_{2} \cdot v\right)^{2}-m^{2}$,

where the last equalities follow from the invariance of the scalar product because, defining the four vector $v_{0}=(1, \mathbf{0})$, one has

$k_{2}^{0}=\left(\mathbf{B}_{v}^{-1} p_{2}\right)^{0}=\left(\mathbf{B}_{v}^{-1} p_{2}\right) \cdot v_{0}=p_{2} \cdot\left(\mathbf{B}_{v} v_{0}\right)=p_{2} \cdot v$.

What is missing are the three-dimensional scalar products like 


$$
\mathbf{k}_{2} \cdot \mathbf{k}_{3}=\left(\overrightarrow{\mathbf{B}_{v}^{-1} p_{2}}\right) \cdot\left(\overrightarrow{\mathbf{B}_{v}^{-1} p_{3}}\right)=\left(\mathbf{B}_{v}^{-1} p_{2}\right)^{0}\left(\mathbf{B}_{v}^{-1} p_{3}\right)^{0}-\left(\mathbf{B}_{v}^{-1} p_{2}\right) \cdot\left(\mathbf{B}_{v}^{-1} p_{3}\right),
$$

and using relations (B27) and the invariance of the scalar product, we have

$$
\mathbf{k}_{2} \cdot \mathbf{k}_{3}=\left(p_{2} \cdot v\right)\left(p_{3} \cdot v\right)-\left(p_{2} \cdot p_{3}\right) .
$$

Finally, we have the Lorentz scalar wave function,

$$
\varphi\left(\mathbf{k}_{2}, \mathbf{k}_{3}\right)=\psi\left(\left(p_{2} \cdot v\right)^{2}-m^{2},\left(p_{3} \cdot v^{\prime}\right)^{2}-m^{2},\left(p_{2} \cdot v\right)\left(p_{3} \cdot v\right)-\left(p_{2} \cdot p_{3}\right)\right),
$$

and similarly, for $\varphi\left(\mathbf{k}_{2}^{\prime}, \mathbf{k}_{3}^{\prime}\right)$.

Finally the baryon Isgur-Wise function writes in the explicit Lorentz invariant form,

$$
\begin{aligned}
\xi_{\Lambda}\left(v \cdot v^{\prime}\right)= & \int \frac{d \mathbf{p}_{2}}{(2 \pi)^{3}} \frac{1}{p_{2}^{0}} \frac{d \mathbf{p}_{3}}{(2 \pi)^{3}} \frac{1}{p_{3}^{0}} \sqrt{\left(p_{2} \cdot v\right)\left(p_{3} \cdot v\right)\left(p_{2} \cdot v^{\prime}\right)\left(p_{3} \cdot v^{\prime}\right)} \\
& \times \psi\left(\left(p_{2} \cdot v^{\prime}\right)^{2}-m^{2},\left(p_{3} \cdot v^{\prime}\right)^{2}-m^{2},\left(p_{2} \cdot v^{\prime}\right)\left(p_{3} \cdot v^{\prime}\right)-\left(p_{2} \cdot p_{3}\right)\right)^{*} \\
& \times \psi\left(\left(p_{2} \cdot v\right)^{2}-m^{2},\left(p_{3} \cdot v\right)^{2}-m^{2},\left(p_{2} \cdot v\right)\left(p_{3} \cdot v\right)-\left(p_{2} \cdot p_{3}\right)\right) \\
& \times \frac{\left[m^{2}\left(1+v \cdot v^{\prime}\right)+m\left(v+v^{\prime}\right) \cdot\left(p_{2}+p_{3}\right)+\left(p_{2} \cdot v\right)\left(p_{3} \cdot v^{\prime}\right)+\left(p_{3} \cdot v\right)\left(p_{2} \cdot v^{\prime}\right)+\left(p_{2} \cdot p_{3}\right)\left(1-v \cdot v^{\prime}\right)\right]}{2 \sqrt{\left(p_{2} \cdot v+m\right)\left(p_{3} \cdot v+m\right)\left(p_{2} \cdot v^{\prime}+m\right)\left(p_{3} \cdot v^{\prime}+m\right)}} .
\end{aligned}
$$

\section{APPENDIX C: THE Q POINTLIKE-DIQUARK IW FUNCTIONS IN THE BT SCHEME}

\section{The elastic IW function}

This case is much simpler than the three-quark one $Q q q$, because the diquark is in a $S=0, L=0$ state, and there are no Wigner rotations on the spectator diquark.

The matrix element reads

$$
\left\langle\mathbf{P}^{\prime}, \mu^{\prime}|O| \mathbf{P}, \mu\right\rangle=\int \frac{d \mathbf{p}_{2}}{(2 \pi)^{3}} \sqrt{\frac{\sum_{j} p_{j}^{\prime 0} \sum_{k} p_{k}^{0}}{M_{0}^{\prime} M_{0}}} \prod_{i=1}^{2} \sqrt{\frac{k_{i}^{\prime 0} k_{i}^{0}}{p_{i}^{\prime 0} p_{i}^{0}}} \varphi^{\prime}\left(\mathbf{k}_{2}^{\prime}\right)^{*} \varphi\left(\mathbf{k}_{2}\right) \operatorname{Tr}\left[\bar{\chi}^{\left(\mu^{\prime}\right)+} D\left(\mathbf{R}_{1}^{\prime-1}\right) O\left(\mathbf{p}_{1}^{\prime}, \mathbf{p}_{1}\right) D\left(\mathbf{R}_{1}\right) \chi^{(\mu)}\right],
$$

that gives, in the heavy quark limit,

$$
\left\langle\mathbf{P}^{\prime}, \mu^{\prime}|O| \mathbf{P}, \mu\right\rangle=\frac{1}{\sqrt{v^{0} v^{\prime 0}}}\left(\bar{\chi}_{v^{\prime}}^{\left(\mu^{\prime}\right)+} O \chi_{v}^{(\mu)}\right) \int \frac{d \mathbf{p}_{2}}{(2 \pi)^{3}} \frac{1}{p_{2}^{0}} \sqrt{\left(p_{2} \cdot v\right)\left(p_{2} \cdot v^{\prime}\right)} \varphi^{\prime}\left(\mathbf{k}_{2}^{\prime}\right)^{*} \varphi\left(\mathbf{k}_{2}\right),
$$

corresponding to the simple expression of the IW function,

$$
\xi_{\Lambda}\left(v \cdot v^{\prime}\right)=\int \frac{d \mathbf{p}_{2}}{(2 \pi)^{3}} \frac{1}{p_{2}^{0}} \sqrt{\left(p_{2} \cdot v\right)\left(p_{2} \cdot v^{\prime}\right)} \varphi^{\prime}\left(\mathbf{k}_{2}^{\prime}\right)^{*} \varphi\left(\mathbf{k}_{2}\right),
$$

that is covariant because $\mathbf{k}_{2}^{2}=\left(p_{2} \cdot v\right)^{2}-m^{2}, \mathbf{k}_{2}^{\prime 2}=\left(p_{2} \cdot v^{\prime}\right)^{2}-m^{2}$, where $m$ is the diquark mass, and is correctly normalized, $\xi_{\Lambda}(1)=1$.

\section{The inelastic $L=0 \rightarrow L=1$ IW function}

$$
\begin{aligned}
\left\langle\mathbf{P}^{\prime}, \mu^{\prime}|O| \mathbf{P}, \mu\right\rangle= & \int \frac{d \mathbf{p}_{2}}{(2 \pi)^{3}} \sqrt{\frac{\sum_{j} p_{j}^{\prime 0} \sum_{k} p_{k}^{0}}{M_{0}^{\prime} M_{0}}} \prod_{i=1}^{2} \sqrt{\frac{k_{i}^{\prime 0} k_{i}^{0}}{p_{i}^{\prime 0} p_{i}^{0}}} \varphi\left(\mathbf{k}_{2}\right) \\
& \times \sum_{s_{1}^{\prime}, s_{1}} \varphi_{s_{1}^{\prime}}^{\prime\left(\mu^{\prime}\right) *}\left(\mathbf{k}_{2}^{\prime}\right)\left[\bar{\chi}^{\left(\mu^{\prime}\right)+} D\left(\mathbf{R}_{1}^{\prime-1}\right) O\left(\mathbf{p}_{1}^{\prime}, \mathbf{p}_{1}\right) D\left(\mathbf{R}_{1}\right) \chi^{(\mu)}\right]_{s_{1}^{\prime} s_{1}} \varphi_{s_{1}}^{(\mu)}\left(\mathbf{k}_{2}\right),
\end{aligned}
$$


where

$$
\begin{aligned}
\varphi_{s_{1}}^{(\mu)}\left(\mathbf{k}_{2}\right) & =\chi_{s_{1}}^{(\mu)} \varphi\left(\mathbf{k}_{2}\right) \\
\varphi_{s_{1}^{\prime}}^{\prime\left(\mu^{\prime}\right) *}\left(\mathbf{k}_{2}^{\prime}\right) & =\sum_{m^{\prime}}\left\langle 1 \mu^{\prime}-m^{\prime}, \frac{1}{2} m^{\prime} \mid J \mu^{\prime}\right\rangle Y_{1}^{\mu^{\prime}-m} \chi_{s_{1}^{\prime}}^{\left(m^{\prime}\right)},
\end{aligned}
$$

where $J=\frac{1}{2}$ or $J=\frac{3}{2}$.

The sum over the Clebsch-Gordan coefficients can be written as

$$
\begin{aligned}
\sum_{m^{\prime}}\left\langle 1 \mu^{\prime}-m^{\prime}, \frac{1}{2} m^{\prime} \mid J \mu^{\prime}\right\rangle Y_{1}^{\mu^{\prime}-m} \chi^{\left(m^{\prime}\right)} & =\frac{1}{\sqrt{4 \pi}} \frac{1}{\left|\mathbf{k}_{2}^{\prime}\right|} \sum_{m^{\prime}}(-1)^{\frac{1}{2}+m^{\prime}}\left[\sigma^{\left(\mu^{\prime}-m^{\prime}\right)} i \sigma_{2}\right]_{\mu^{\prime},-m^{\prime}} \chi^{\left(m^{\prime}\right)}\left(\mathbf{k}_{2}^{\prime}\right)^{\mu^{\prime}-m^{\prime}} \\
& =-\frac{1}{\sqrt{4 \pi}} \frac{1}{\left|\mathbf{k}_{2}^{\prime}\right|}\left(\sigma . \mathbf{k}_{2}^{\prime}\right) \chi^{\left(\mu^{\prime}\right)} .
\end{aligned}
$$

Passing now to the $4 \times 4$ matrix formulation and taking the heavy quark limit, one finds, after some algebra,

$$
\begin{aligned}
\left\langle\mathbf{P}^{\prime}, \mu^{\prime}|O| \mathbf{P}, \mu\right\rangle= & \frac{1}{\sqrt{v^{0} v^{0}}} \frac{1}{4} \int \frac{d \mathbf{p}_{2}}{(2 \pi)^{3}} \frac{1}{p_{2}^{0}} \varphi^{\prime}\left(\mathbf{k}_{2}^{\prime}\right)^{*} \varphi\left(\mathbf{k}_{2}\right) \\
& \times \frac{1}{\sqrt{\left(p_{2} \cdot v^{\prime}\right)^{2}-m^{2}}} \bar{\chi}_{v^{\prime}}^{\left(\mu^{\prime}\right)}\left\{\left[\not \not_{2}-\left(p_{2} \cdot v^{\prime}\right) \not \not^{\prime}\right] \gamma_{5}\left(1+\not \not^{\prime}\right) O(1+\not h)\right\} \chi_{v}^{(\mu)} .
\end{aligned}
$$

Particularizing to $J=\frac{1}{2}$ and identifying to the HQET matrix element defining the Isgur-Wise function $\sigma(w)$ [35],

$$
\left\langle\mathbf{P}^{\prime}, \mu^{\prime}|O| \mathbf{P}, \mu\right\rangle=\frac{\sigma(w)}{\sqrt{3}}\left[\bar{\chi}_{v^{\prime}}^{\left(\mu^{\prime}\right)} \gamma_{5}(\not p+w) O \chi_{v}^{(\mu)}\right],
$$

one finds

$$
\sigma(w)=\frac{\sqrt{3}}{w^{2}-1} \int \frac{d \mathbf{p}_{2}}{(2 \pi)^{3}} \frac{1}{p_{2}^{0}} \sqrt{\left(p_{2} \cdot v\right)\left(p_{2} \cdot v^{\prime}\right)} \varphi^{\prime}\left(\mathbf{k}_{2}^{\prime}\right)^{*} \varphi\left(\mathbf{k}_{2}\right) \frac{p_{2} \cdot\left(v-w v^{\prime}\right)}{\sqrt{\left.\left(p_{2} \cdot v^{\prime}\right)^{2}-m^{2}\right)^{2}-m^{2}}} .
$$

\section{APPENDIX D: BING CHEN et al. $L=0$ AND $L=1$ WAVE FUNCTIONS IN THE QUARK-DIQUARK MODEL}

In an expansion in terms of $L=0$ and $L=1$ on harmonic oscillator bases,

$$
\begin{gathered}
\varphi_{0}^{(n)}(\mathbf{p})=(-1)^{n}(4 \pi)^{3 / 4} 2^{n} \sqrt{\frac{(n !)^{2}}{(2 n+1) !}} \frac{1}{\beta^{3 / 2}} L_{n}^{1 / 2}\left(\frac{\mathbf{p}^{2}}{\beta^{2}}\right) \exp \left(-\frac{\mathbf{p}^{2}}{2 \beta^{2}}\right) \\
\varphi_{1}^{(n)}(\mathbf{p})=(-1)^{n}(4 \pi)^{3 / 4} 2^{n+1} \sqrt{\frac{n !(n+1) !}{(2 n+3) !}} \frac{1}{\beta^{5 / 2}}|\mathbf{p}| L_{n}^{3 / 2}\left(\frac{\mathbf{p}^{2}}{\beta^{2}}\right) \exp \left(-\frac{\mathbf{p}^{2}}{2 \beta^{2}}\right),
\end{gathered}
$$

the ground state wave function reads, with the calculation of the wave function in the heavy quark limit,

$$
\begin{aligned}
\varphi_{0}(\mathbf{p})= & -0.9940325 \varphi_{0}^{(0)}(\mathbf{p})-8.5672485 \times 10^{-3} \varphi_{0}^{(1)}(\mathbf{p}) \\
& -9.9527270 \times 10^{-2} \varphi_{0}^{(2)}(\mathbf{p})-2.4497384 \times 10^{-2} \varphi_{0}^{(3)}(\mathbf{p}) \\
& -2.7361497 \times 10^{-2} \varphi_{0}^{(4)}(\mathbf{p})-1.4908912 \times 10^{-2} \varphi_{0}^{(5)}(\mathbf{p}) \\
& -1.2411494 \times 10^{-2} \varphi_{0}^{(6)}(\mathbf{p})-9.4764605 \times 10^{-3} \varphi_{0}^{(7)}(\mathbf{p}) \\
& -6.3898186 \times 10^{-3} \varphi_{0}^{(8)}(\mathbf{p})-8.0367858 \times 10^{-3} \varphi_{0}^{(9)}(\mathbf{p}),
\end{aligned}
$$


and the $L=1$ wave function,

$$
\begin{aligned}
\varphi_{1}(\mathbf{p})= & 0.9482319 \varphi_{1}^{(0)}(\mathbf{p})-0.2740721 \varphi_{1}^{(1)}(\mathbf{p}) \\
& +0.1497750 \varphi_{1}^{(2)}(\mathbf{p})-4.7684737 \times 10^{-2} \varphi_{1}^{(3)}(\mathbf{p}) \\
& +3.0210067 \times 10^{-2} \varphi_{1}^{(4)}(\mathbf{p})-7.8150993 \times 10^{-3} \varphi_{1}^{(5)}(\mathbf{p}) \\
& +7.4121789 \times 10^{-3} \varphi_{1}^{(6)}(\mathbf{p})-5.9317378 \times 10^{-4} \varphi_{1}^{(7)}(\mathbf{p}) \\
& +2.1176776 \times 10^{-3} \varphi_{1}^{(8)}(\mathbf{p})+9.3134667 \times 10^{-4} \varphi_{1}^{(9)}(\mathbf{p})
\end{aligned}
$$

\section{APPENDIX E: HELICITY AMPLITUDES AND OBSERVABLES}

The expressions for the helicity amplitudes and observables as formulated by Gutsche et al. [28] are summarized here. In terms of $V-A$ chiral helicity amplitudes,

$$
H_{\lambda_{2}, \lambda_{W}}=H_{\lambda_{2}, \lambda_{W}}^{V}-H_{\lambda_{2}, \lambda_{W}}^{A}
$$

( $\lambda_{2}$ : helicity of the final $\Lambda_{c}, \lambda_{W}$ : helicity of the final virtual $W$ ).

Gutsche et al. define the following bilinears in terms of helicity amplitudes:

$$
\begin{aligned}
& \mathcal{H}_{U}=\left|H_{+\frac{1}{2}+1}\right|^{2}+\left|H_{-\frac{1}{2}-1}\right|^{2}, \mathcal{H}_{P}=\left|H_{+\frac{1}{2}+1}\right|^{2}-\left|H_{-\frac{1}{2}-1}\right|^{2} \\
& \mathcal{H}_{L}=\left|H_{+\frac{1}{2} 0}\right|^{2}+\left|H_{-\frac{1}{2}}\right|^{2}, \mathcal{H}_{L_{P}}=\left|H_{+\frac{1}{2} 0}\right|^{2}-\left|H_{-\frac{1}{2} 0}\right|^{2} \\
& \mathcal{H}_{S}=\left|H_{+\frac{1}{2} t}\right|^{2}+\left|H_{-\frac{1}{2} t}\right|^{2}, \quad \mathcal{H}_{S_{P}}=\left|H_{+\frac{1}{2} t}\right|^{2}-\left|H_{-\frac{1}{2} t}\right|^{2} \\
& \mathcal{H}_{L T}=\operatorname{Re}\left(H_{+\frac{1}{2}+1} H_{-\frac{1}{2} 0}^{\dagger}+H_{+\frac{1}{2} 0} H_{-\frac{1}{2}-1}^{\dagger}\right), \quad \mathcal{H}_{L T_{P}}=\operatorname{Re}\left(H_{+\frac{1}{2}+1} H_{-\frac{1}{2} 0}^{\dagger}-H_{+\frac{1}{2} 0} H_{-\frac{1}{2}-1}^{\dagger}\right) \\
& \mathcal{H}_{S T}=\operatorname{Re}\left(H_{+\frac{1}{2}+1} H_{-\frac{1}{2} t}^{\dagger}+H_{+\frac{1}{2}} H_{-\frac{1}{2}-1}^{\dagger}\right), \quad \mathcal{H}_{L T_{P}}=\operatorname{Re}\left(H_{+\frac{1}{2}+1} H_{-\frac{1}{2} t}^{\dagger}-H_{+\frac{1}{2} t} H_{-\frac{1}{2}-1}^{\dagger}\right) \\
& \mathcal{H}_{S L}=\operatorname{Re}\left(H_{+\frac{1}{2} 0} H_{+\frac{1}{2} t}^{\dagger}+H_{-\frac{1}{2} 0} H_{-\frac{1}{2} t}^{\dagger}\right), \quad \mathcal{H}_{S L_{P}}=\operatorname{Re}\left(H_{+\frac{1}{2} 0} H_{+\frac{1}{2} t}^{\dagger}-H_{-\frac{1}{2} 0} H_{-\frac{1}{2} t}^{\dagger}\right),
\end{aligned}
$$

where the left (right) column corresponds to parity conserving (parity violating) quantities, and

$$
\mathcal{H}_{\mathrm{tot}}=\mathcal{H}_{U}+\mathcal{H}_{L}+\delta_{\ell}\left(\mathcal{H}_{U}+\mathcal{H}_{L}+3 \mathcal{H}_{S}\right)
$$

with the dependence on the lepton mass given by

$$
\delta_{\ell}=\frac{m_{\ell}^{2}}{2 q^{2}}
$$

In terms of these quantities, the interesting observables read as below.

Differential rate,

$$
\frac{d \Gamma}{d q^{2}}=\Gamma_{0} \frac{\left(q^{2}-m_{\ell}^{2}\right)^{2}\left|\mathbf{p}_{2}\right|}{M_{1}^{7} q^{2}} \mathcal{H}_{\mathrm{tot}}
$$

where

$$
\Gamma_{0}=\frac{G_{F}^{2}\left|V_{c b}\right|^{2} M_{1}^{5}}{192 \pi^{3}}
$$

Forward-backward asymmetry,

$$
A_{\mathrm{FB}}^{\ell}\left(q^{2}\right)=\frac{d \Gamma(F)-d \Gamma(B)}{d \Gamma(F)+d \Gamma(B)}=-\frac{3}{2} \frac{\mathcal{H}_{P}+4 \delta_{\ell} \mathcal{H}_{S L}}{\mathcal{H}_{\mathrm{tot}}}
$$

Convexity parameter (second derivative of the zenithal angular distribution),

$$
C_{F}\left(q^{2}\right)=\frac{1}{\mathcal{H}_{\mathrm{tot}}} \frac{d^{2} W(\theta)}{d(\cos \theta)^{2}}=\frac{3}{4}\left(1-2 \delta_{\ell}\right) \frac{\mathcal{H}_{U}-2 \mathcal{H}_{L}}{\mathcal{H}_{\mathrm{tot}}}
$$




\section{Longitudinal hadron polarization,}

$$
P_{z}^{h}\left(q^{2}\right)=\frac{\mathcal{H}_{P}+\mathcal{H}_{L_{P}}+\delta_{\ell}\left(\mathcal{H}_{P}+\mathcal{H}_{L_{P}}+3 \mathcal{H}_{S_{P}}\right)}{\mathcal{H}_{\text {tot }}} .
$$

Tranverse hadron polarization,

$$
P_{x}^{h}\left(q^{2}\right)=-\frac{3 \pi}{4 \sqrt{2}} \frac{\mathcal{H}_{L T}-2 \delta_{\ell} \mathcal{H}_{S T_{P}}}{\mathcal{H}_{\mathrm{tot}}}
$$

Longitudinal lepton polarization,

$P_{z}^{\ell}\left(q^{2}\right)=-\frac{\mathcal{H}_{U}+\mathcal{H}_{L}-\delta_{\ell}\left(\mathcal{H}_{U}+\mathcal{H}_{L}+3 \mathcal{H}_{S}\right)}{\mathcal{H}_{\text {tot }}}$.

Transverse lepton polarization,

$$
P_{x}^{\ell}\left(q^{2}\right)=-\frac{3 \pi}{4 \sqrt{2}} \sqrt{\delta_{\ell}} \frac{\mathcal{H}_{P}-2 \mathcal{H}_{S L}}{\mathcal{H}_{\mathrm{tot}}} .
$$

[1] J. P. Lees et al. (BABAR Collaboration), Measurement of an excess of $B^{-} \rightarrow D^{(*)} \tau^{-} \bar{\nu}_{\tau}$ decays and implications for charged Higgs bosons, Phys. Rev. D 88, 072012 (2013).

[2] M. Huschle et al. (Belle Collaboration), Measurement of the branching ratio of $\bar{B} \rightarrow D^{(*)} \tau^{-} \bar{\nu}_{\tau}$ relative to $\bar{B} \rightarrow D^{(*)} \ell^{-} \bar{\nu}_{\ell}$ decays with hadronic tagging at Belle, Phys. Rev. D 92, 072014 (2015); A. Abdesselam et al. (Belle Collaboration), Measurement of the branching ratio of $\bar{B}^{0} \rightarrow D^{*+} \tau^{-} \bar{\nu}_{\tau}$ relative to $\bar{B}^{0} \rightarrow D^{*+} \ell^{-} \bar{\nu}_{\ell}$ decays with a semileptonic tagging method, arXiv:1603.06711.

[3] R. Aaij et al. (LHCb Collaboration), Measurement of the Ratio of Branching Fractions $B\left(\bar{B}^{0} \rightarrow D^{*+} \tau^{-} \bar{\nu}_{\tau}\right) /$ $B\left(\bar{B}^{0} \rightarrow D^{*+} \mu^{-} \bar{\nu}_{\mu}\right)$, Phys. Rev. Lett. 115, 111803 (2015).

[4] Y. Amhis et al. (Heavy Flavor Averaging Group), Averages of $b$ hadron, $c$ hadron, and $\tau$ lepton properties as of winter 2016, http://www.slac.stanford.edu/xorg/hfag/semi/ winter16/winter16.

[5] Y. Sakaki, M. Tanaka, A. Tayduganov, and R. Watanabe, Testing leptoquark models in $\bar{B} \rightarrow D^{(*)} \tau \bar{\nu}$, Phys. Rev. D 88, 094012 (2013).

[6] S. Fajfer, J. Kamenik, and I. Nisandzic, On the $B \rightarrow D^{*} \tau^{-} \nu$ sensitivity to new physics, Phys. Rev. D 85, 094025 (2012).

[7] T. Gutsche, M. Ivanov, J. Koerner, V. Lyubovitskij, P. Santorelli, and N. Habyl, Semileptonic decay $\Lambda_{b} \rightarrow \Lambda_{c}+$ $\tau^{-}+\bar{\nu}_{\tau}$ in the covariant confined quark model, Phys. Rev. D 91, 074001 (2015); Erratum, Phys. Rev. D 91, 119907 (2015).

[8] R. Woloshyn, Semileptonic decays of the $\Lambda_{b}$ baryon, Proc. Sci. Hadron2013 (2013) 203.

[9] S. Shivashankara, W. Wu, and A. Datta, $\Lambda_{b} \rightarrow \Lambda_{c} \tau \bar{\nu}_{\tau}$ decays in the standard model and with new physics, Phys. Rev. D 91, 115003 (2015).

[10] R. Dutta, $\Lambda_{b} \rightarrow\left(\Lambda_{c}, p\right) \tau \bar{\nu}_{\tau}$ decays within the standard model and beyond, Phys. Rev. D 93, 054003 (2016).

[11] R. Faustov and V. Galkin, Semileptonic decays of $\Lambda_{b}$ baryons in the relativistic quark model, Phys. Rev. D 94, 073008 (2016).

[12] X.-Q. Li, Y.-D. Yang, and X. Zhang, $\Lambda_{b} \rightarrow \Lambda_{c} \tau \bar{\nu}_{\tau}$ decay in scalar and vector leptoquark scenarios, J. High Energy Phys. 02 (2017) 068.

[13] W. Detmold, C. Lehner, and S. Meinel, $\Lambda_{b} \rightarrow p \ell^{-} \bar{\nu}_{\ell}$ and $\Lambda_{b} \rightarrow \Lambda_{c} \ell^{-} \bar{\nu}_{\ell}$ form factors from lattice QCD with relativistic heavy quarks, Phys. Rev. D 92, 034503 (2015).
[14] A. Datta, S. Kamali, S. Meinel, and A. Rashed, Phenomenology of $\Lambda_{b} \rightarrow \Lambda_{c} \tau \bar{\nu}_{\tau}$ using lattice QCD calculations, J. High Energy Phys. 08 (2017) 131.

[15] A. Le Yaouanc, L. Oliver, O. Pène, and J.-C. Raynal, Covariant quark model of form-factors in the heavy mass limit, Phys. Lett. B 365, 319 (1996).

[16] V. Morénas, A. Le Yaouanc, L. Oliver, O. Pène, and J.-C. Raynal, $B \rightarrow D^{* *}$ semileptonic decay in covariant quark models à la Bakamjian-Thomas, Phys. Lett. B 386, 315 (1996).

[17] S. Godfrey and N. Isgur, Mesons in a relativized quark model with chromodynamics, Phys. Rev. D 32, 189 (1985).

[18] V. Morénas, A. Le Yaouanc, L. Oliver, O. Pène, and J.-C. Raynal, Quantitative predictions for $B$ semileptonic decays into $D, D^{*}$ and the orbitally excited $D^{* *}$ in quark models à la Bakamjian-Thomas, Phys. Rev. D 56, 5668 (1997).

[19] C. Albertus, J. Amaro, E. Hernandez, and J. Nieves, Charmed and bottom baryons: A Variational approach based on heavy quark symmetry, Nucl. Phys. A740, 333 (2004); C. Albertus, E. Hernandez, and J. Nieves, Combined nonrelativistic constituent quark model and heavy quark effective theory study of semileptonic decays of $\Lambda_{b}$ and $\Xi_{b}$ baryons, Phys. Rev. D 71, 014012 (2005).

[20] M. Pervin, W. Roberts, and S. Capstick, Semileptonic decays of heavy lambda baryons in a quark model, Phys. Rev. C 72, 035201 (2005).

[21] R. Aaij et al. (LHCb Collaboration), Measurement of the shape of the $\Lambda_{b}^{0} \rightarrow \Lambda_{c}^{+} \mu^{-} \bar{\nu}_{\mu}$ differential decay rate, Phys. Rev. D 96, 112005 (2017).

[22] V. Morénas, A. Le Yaouanc, L. Oliver, O. Pene, and J.-C. Raynal, Slope of the Isgur-Wise function in the heavy mass limit of quark models a la Bakamjian-Thomas, Phys. Lett. B 408, 357 (1997).

[23] D. Merten, R. Ricken, M. Koll, B. Metsch, and H. Petry, Weak decays of heavy mesons in a covariant quark model, Eur. Phys. J. A 13, 477 (2002); D. Merten, H.-R. Petry, F. Klein, U.-G. Meißner, and U. Thoma, EPJ Web Conf. 134, 02005 (2017).

[24] S. Capstick and N. Isgur, Baryons in a relativized quark model with chromodynamics, Phys. Rev. D 34, 2809 (1986); AIP Conf. Proc. 132, 267 (1985).

[25] F. Cardarelli and S. Simula, Isgur-Wise form-factors of heavy baryons within a light front constituent quark model, Phys. Lett. B 421, 295 (1998); Analysis of the exclusive 
semileptonic decay $\Lambda_{b} \rightarrow \Lambda_{c}+\ell+\bar{\nu}$ within a light front constituent quark model, Nucl. Phys. A663, 931 (2000); Analysis of the $\Lambda_{b} \rightarrow \Lambda_{c}+\ell+\bar{\nu}$ decay within a light front constituent quark model, Phys. Rev. D 60, 074018 (1999).

[26] D. Ebert, R. Faustov, and V. Galkin, Spectroscopy and Regge trajectories of heavy baryons in the relativistic quarkdiquark picture, Phys. Rev. D 84, 014025 (2011); Masses of excited heavy baryons in the relativistic quark model, Phys. Lett. B 659, 612 (2008); Semileptonic decays of heavy baryons in the relativistic quark model, Phys. Rev. D 73, 094002 (2006).

[27] B. Chen, K.-W. Wei, X. Liu, and T. Matsuki, Low-lying charmed and charmed-strange baryon states, Eur. Phys. J. C 77, 154 (2017).

[28] T. Gutsche, M. Ivanov, J. Koerner, V. Lyubovitskij, P. Santorelli, and C.-T. Tran, Analyzing lepton flavor universality in the decays $\Lambda_{b} \rightarrow \Lambda_{c}^{(*)}\left(\frac{1}{2}^{ \pm}, \frac{3}{2}^{-}\right)+\ell+\bar{\nu}_{\ell}$, Phys. Rev. D 98, 053003 (2018).

[29] A. Le Yaouanc, L. Oliver, and J.-C. Raynal, Bound on the curvature of the Isgur-Wise function of the baryon semileptonic decay $\Lambda_{b} \rightarrow \Lambda_{c} \ell \bar{\nu}_{\ell}$, Phys. Rev. D 79, 014023 (2009).

[30] A. Le Yaouanc, L. Oliver, and J.-C. Raynal, Isgur-Wise functions and unitary representations of the Lorentz group: The baryon case $j=0$, Phys. Rev. D 80, 054006 (2009).

[31] K. C. Bowler, R. D. Kenway, L. Lellouch, J. Nieves, O. Oliveira, D. G. Richards, C. T. Sachrajda, N. Stella, and P. Ueberholz (UKQCD Collaboration), First lattice study of semileptonic decays of $\Lambda_{b}$ and $\Xi_{b}$ baryons, Phys. Rev. D 57, 6948 (1998).

[32] S. Gottlieb and S. Tamhankarn, A lattice study of $\Lambda_{b}$ semileptonic decay, Nucl. Phys. B, Proc. Suppl. 119, 644 (2003).

[33] M. Luke, Effects of subleading operators in the heavy quark effective theory, Phys. Lett. B 252, 447 (1990).

[34] C. Bourrely, I. Caprini, and L. Lellouch, Model-independent description of $B \rightarrow \pi \ell \nu$ decays and a determination of $|V(u b)|$, Phys. Rev. D 79, 013008 (2009); Erratum, Phys. Rev. D 82, 099902 (2010).

[35] A. Leibovich and I. Stewart, Semileptonic Lambda(b) decay to excited Lambda(c) baryons at order Lambda(QCD)/m(Q), Phys. Rev. D 57, 5620 (1998).

[36] Y. Jia, F. Jugeau, and L. Oliver, New results on the baryon decay $\Lambda_{b} \rightarrow \Lambda_{c} \ell \nu$ in heavy quark effective theory, Phys. Rev. D 86, 014002 (2012).
[37] I. Bigi, M. Shifman, and N. Uraltsev, Aspects of heavy quark theory, Annu. Rev. Nucl. Part. Sci. 47, 591 (1997).

[38] M. Jarfi, O. Lazrak, A. Le Yaouanc, L. Oliver, O. Pène, and J.-C. Raynal, Decays of b mesons into baryon - anti-baryon, Phys. Rev. D 43, 1599 (1991).

[39] H. Hernandez, J. Nieves, and J. M. Verde-Velasco, Test of the heavy quark-light diquark approximation for baryons with a heavy quark, Phys. Lett. B 666, 150 (2008).

[40] J. Green, J. Negele, M. Engelhardt, and P. Varilly, Spatial diquark correlations in a hadron, Proc. Sci. LATTICE2010 (2010) 140 [arXiv:1012.2353].

[41] R. Fukuda and Ph. de Forcrand, Searching for evidence of diquark states using lattice QCD simulations, Proc. Sci. LATTICE2016 (2017) 121; C. Alexandrou, Ph. de Forcrand, and B. Lucini, Evidence for Diquarks in Lattice QCD, Phys. Rev. Lett. 97, 222002 (2006).

[42] X.-Y. Guo and T. Muta, Isgur-wise function for $\Lambda_{b} \rightarrow \Lambda_{c}$ in B-S approach, Phys. Rev. D 54, 4629 (1996).

[43] N. Isgur, M. Wise, and M. Youssefmir, Excited charm baryons in semileptonic Lambda(b) decay and their contribution to a Bjorken sum rule, Phys. Lett. B 254, 215 (1991).

[44] T. Gutsche, N. Habyl, M. Ivanov, J. Koerner, V. Lyubovitskij, and P. Santorelli, Physical observables in the decay $\Lambda_{b} \rightarrow \Lambda_{c}(\rightarrow \Lambda \pi)+\tau+\bar{\nu}_{\tau}$, Int. J. Mod. Phys. Conf. Ser. 39, 1560112 (2015); Semileptonic decay $\Lambda_{b} \rightarrow \Lambda_{c}(\rightarrow \Lambda \pi)+\tau+\bar{\nu}_{\tau}$, Phys. Rev. D 91, 074001 (2015); Erratum, Phys. Rev. D 91, 119907 (2015).

[45] T. Gutsche, M. Ivanov, J. Koerner, V. Lyubovitskij, and P. Santorelli, Theoretical description of the decays $\Lambda_{b} \rightarrow$ $\Lambda^{(*)}\left(\frac{1}{2}^{ \pm}, \frac{3}{2}^{ \pm}\right)+J / \psi$, Phys. Rev. D 96, 013003 (2017).

[46] F. Bernlochner, Z. Ligeti, D. Robinson, and W. Sutcliffe, New Predictions for $\Lambda_{b} \rightarrow \Lambda_{c}$ Semileptonic Decays and Tests of Heavy Quark Symmetry, Phys. Rev. Lett. 121, 202001 (2018); Precise predictions for $\Lambda_{b} \rightarrow \Lambda_{c}$ semileptonic decays, Phys. Rev. D 99, 055008 (2019).

[47] P. Boer, M. Bordone, E. Graverini, P. Owen, M. Rotondo, and D. van Dyk, Testing lepton flavour universality in semileptonic $\Lambda_{b} \rightarrow \Lambda_{c}^{*}$ decays, J. High Energy Phys. 06 (2018) 155.

[48] A. Falk and M. Neubert, Second order power corrections in the heavy quark effective theory II. Baryon form factors, Phys. Rev. D 47, 2982 (1993). 